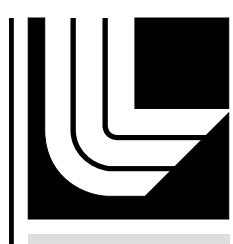

LAWRENCE LIVERMORE N A TIO N A L LABORATORY

Scattering and; Delay, Scale, and Sum Migration

S. K. Lehman

July 15, 2011 
This document was prepared as an account of work sponsored by an agency of the United States government. Neither the United States government nor Lawrence Livermore National Security, LLC, nor any of their employees makes any warranty, expressed or implied, or assumes any legal liability or responsibility for the accuracy, completeness, or usefulness of any information, apparatus, product, or process disclosed, or represents that its use would not infringe privately owned rights. Reference herein to any specific commercial product, process, or service by trade name, trademark, manufacturer, or otherwise does not necessarily constitute or imply its endorsement, recommendation, or favoring by the United States government or Lawrence Livermore National Security, LLC. The views and opinions of authors expressed herein do not necessarily state or reflect those of the United States government or Lawrence Livermore National Security, LLC, and shall not be used for advertising or product endorsement purposes.

This work performed under the auspices of the U.S. Department of Energy by Lawrence Livermore National Laboratory under Contract DE-AC52-07NA27344. 


\title{
Scattering and; Delay, Scale, and Sum Migration
}

\author{
Sean K. Lehman
}

July 12, 2011
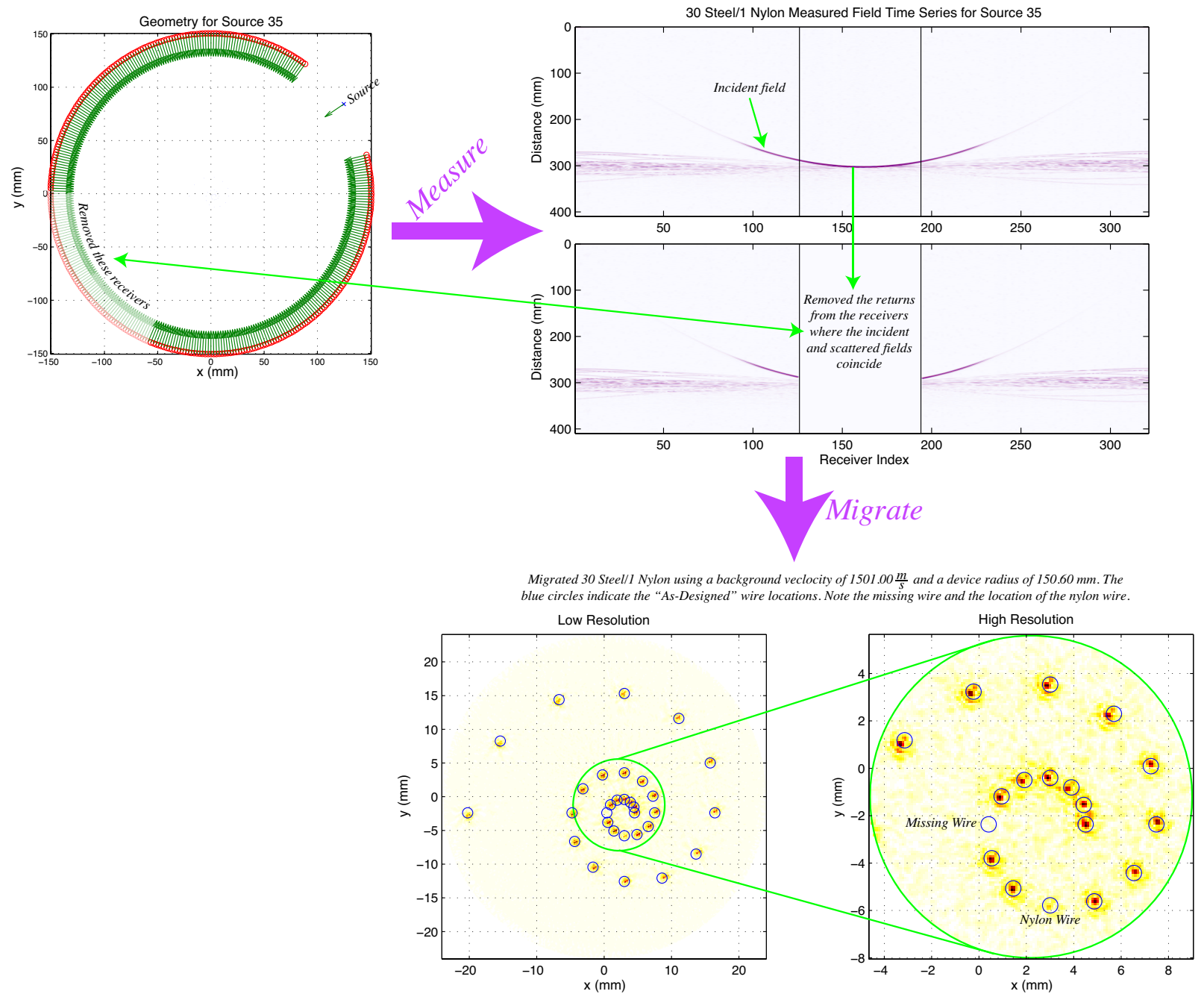


\section{Contents}

1 Introduction $\quad 1$

2 A Variable Bestiary 2

3 Migration in a Nutshell $\quad 4$

3.1 A Brief, Graphical Description of Resolution Limit . . . . . . . . . . . . . . . . . . . . . 9

4 Incident Field Propagation $\quad 21$

5 Scattered Field Propagation $\quad 22$

6 Born Approximation $\quad 24$

6.1 Scattered Field Resulting from a Point Source . . . . . . . . . . . . . . . . . . . . . . . . . . 24

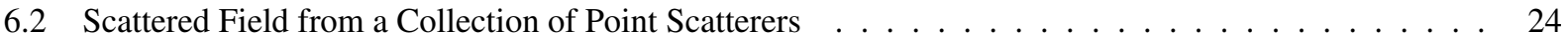

7 Full Scattering via Foldy-Lax $\quad 26$

7.1 Stability Analysis of Foldy-Lax . . . . . . . . . . . . . . . . . . . . . 27

8 Delay, Scale, \& Sum Beamforming: Migration 28

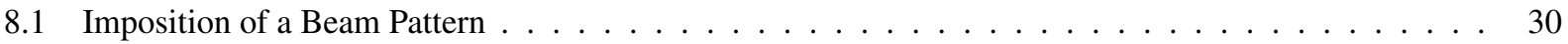

8.2 Migration in a Planar Layer Medium . . . . . . . . . . . . . . . . . . . 30

9 Examples 33

9.1 Wire Phantom . . . . . . . . . . . . . . . . . . . . . . . 33

9.2 Aluminum/Copper Planar Multilayer . . . . . . . . . . . . . . . . . . . . . . . 37

9.3 Ground Penetrating Radar . . . . . . . . . . . . . . . . . . . . . . . . . 37

9.4 Aluminum Block as a Model for Offset Vertical Seismic Profiling . . . . . . . . . . . . . . . 37

A Standard Fourier Transforms 4

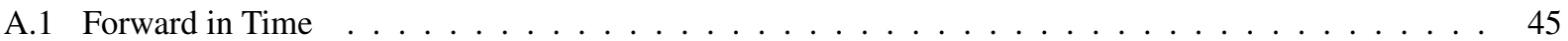

A.2 Inverse in Time . . . . . . . . . . . . . . . . . . . . . . . . . . . 45

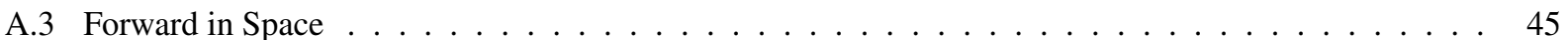

A.4 Inverse in Space . . . . . . . . . . . . . . . . . . . . . . . . . . . . 45

A.5 Planar Fourier Transform Pair $\ldots \ldots \ldots \ldots \ldots \ldots \ldots$

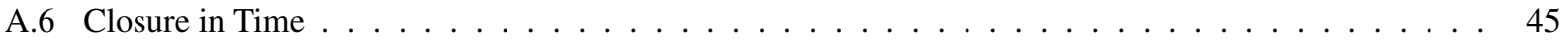

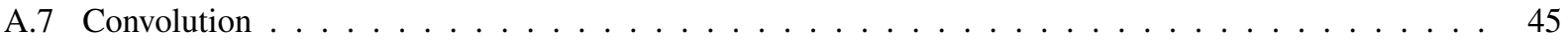

B Measurement System Operating Modes $\quad 47$

$\begin{array}{lll}\text { C Three Dimensional Planar Layer Ray Trace } & 48\end{array}$

C.1 Addressing Numerical Concerns $\ldots \ldots \ldots \ldots \ldots \ldots$

$\begin{array}{lr}\text { D Hilbert Transform } & 51\end{array}$

E Problem Solving Methodology $\quad 52$

F MATLAB Codes $\quad \mathbf{5 3}$

F.1 Migration Parameter Codes . . . . . . . . . . . . . . . . . . . . . . . . . . . . . . . . 54

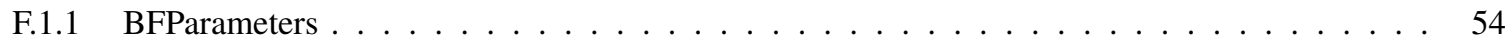

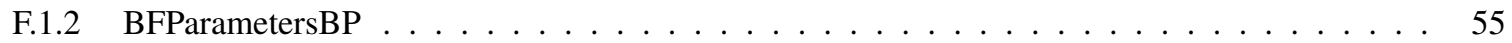

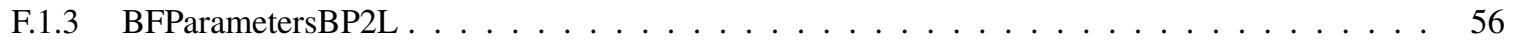


F.2 Migration Computation Codes $\ldots \ldots \ldots \ldots \ldots \ldots \ldots \ldots$

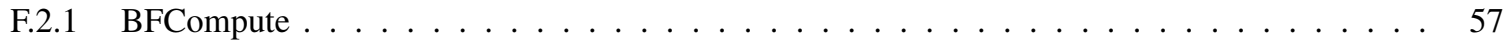

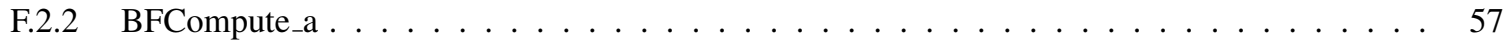

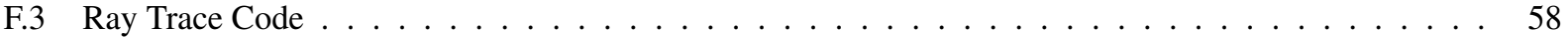

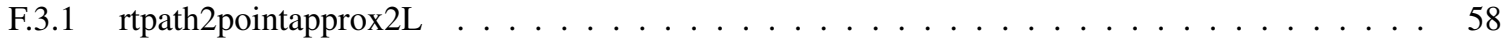




\section{Introduction}

How do we see? What is the mechanism?

Consider standing in an open field on a clear sunny day. In the field are a yellow dog and a blue ball. From a wavebased remote sensing point of view the sun is a source of radiation. It is a broadband electromagnetic source which, for the purposes of this introduction, only the visible spectrum is considered (approximately 390 to 750 nanometers or 400 to 769 TeraHertz). The source emits an incident field into the known background environment which, for this example, is free space. The incident field propagates until it strikes an object or target, either the yellow dog or the blue ball. The interaction of the incident field with an object results in a scattered field. The scattered field arises from a mis-match between the background refractive index, considered to be unity, and the scattering object refractive index ("yellow" for the case of the dog, and "blue" for the ball). This is also known as an impedance mis-match. The scattering objects are referred to as secondary sources of radiation, that radiation being the scattered field which propagates until it is measured by the two receivers known as "eyes". The eyes focus the measured scattered field to form images which are processed by the "wetware" of the brain for detection, identification, and localization. When time series representations of the measured scattered field are available, the image forming focusing process can be mathematically modeled by delayed, scaled, and summed migration.

This concept of optical propagation, scattering, and focusing have one-to-one equivalents in the acoustic realm. This document is intended to present the basic concepts of scalar scattering and migration used in wide band wavebased remote sensing and imaging. The terms beamforming and (delayed, scaled, and summed) migration are used interchangeably but are to be distinguished from the narrow band (frequency domain) beamforming to determine the direction of arrival of a signal [1,2], and seismic migration [3, 4, 5] in which wide band time series are shifted but not to form images per se.

Section 3 presents a mostly graphically-based motivation and summary of delay, scale, and sum beamforming. The model for incident field propagation in free space is derived in Section 4 under specific assumptions. General object scattering is derived in Section 5 and simplified under the Born approximation in Section 6. The model of this section serves as the basis in the derivation of time-domain migration. The Foldy-Lax, full point scatterer scattering, method is derived in Section 7. With the previous forward models in hand, delay, scale, and sum beamforming is derived in Section 8. Finally, proof-of-principle experiments are present in Section 9. 


\section{A Variable Bestiary}

\begin{tabular}{|c|c|}
\hline$\alpha$ & $\begin{array}{l}\text { The range correction applied to compensate for the geometric amplitude attenuation. This } \\
\text { is usually the " } 1 / r^{2} \text { ", range squared, correction (in three dimensions) but may depend upon } \\
\text { the electronics of the measurement system. }\end{array}$ \\
\hline$\alpha_{m \mathrm{fn} n}$ & $\begin{array}{l}\text { The attenuation from the } n \text {-th source to a focal point, and from the focal point to the } m \text {-th } \\
\text { receiver: }\left|\mathbf{R}_{m}^{\text {rrv }}-\mathbf{r}_{\mathrm{f}}\right| \times\left|\mathbf{r}_{\mathrm{f}}-\mathbf{R}_{n}^{\mathrm{src}}\right| \text {. }\end{array}$ \\
\hline$\Delta t$ & The time series sample interval. \\
\hline & Wavelength at the peak pulse temporal frequency, $\lambda_{0}=c_{0} / f_{0}$. \\
\hline$\psi^{\text {inc }}\left(\mathbf{r}, \mathbf{r}^{\prime}, t\right)$ & The incident field evaluated at $\mathbf{r}$ due to a source at $\mathbf{r}^{\prime}$. \\
\hline$\psi^{\text {scat }}\left(\mathbf{r}, \mathbf{r}^{\prime}, t\right)$ & The scattered field evaluated at $\mathbf{r}$ due to a source at $\mathbf{r}^{\prime}$. \\
\hline$\psi^{\text {tot }}\left(\mathbf{r}, \mathbf{r}^{\prime}, t\right)$ & $\begin{array}{l}\text { The total field evaluated at } \mathbf{r} \text { due to a source at } \mathbf{r}^{\prime} \text {. The total field is the sum of the incident } \\
\text { and scattered fields. }\end{array}$ \\
\hline$\tau_{j}$ & The complex scattering amplitude of the $j$-th point scatterer. \\
\hline$\omega$ & Radial frequency, $\omega=2 \pi f$. \\
\hline$c_{0}$ & The background propagation velocity. \\
\hline$c(\mathbf{r})$ & A velocity distribution. \\
\hline$e_{n}(t)$ & The excitement voltage which drives the $n$-th source transducer. \\
\hline & Peak pulse temporal frequency, $f_{0}=c_{0} / \lambda_{0}$. \\
\hline$G_{0}(\mathbf{r}, t), G_{0}\left(\mathbf{r}, t ; \mathbf{r}^{\prime}, t^{\prime}\right)$ & $\begin{array}{l}\text { The background Green function. Generally the zero subscript is used to indicate the free- } \\
\text { space Green function. The un-primed variables represent the observer space-time location; } \\
\text { the primed variables indicate the source space-time location. The latter are frequently } \\
\text { omitted but implicitly assumed. }\end{array}$ \\
\hline$G_{1}(\mathbf{r}, t), G_{1}\left(\mathbf{r}, t ; \mathbf{r}^{\prime}, t^{\prime}\right)$ & $\begin{array}{l}\text { The background Green function in a two layer medium. The un-primed variables repre- } \\
\text { sent the observer space-time location; the primed variables indicate the source space-time } \\
\text { location. The latter are frequently omitted but implicitly assumed. }\end{array}$ \\
\hline$k_{0}$ & Wavenumber at peak temporal frequency, $k_{0}=2 \pi / \lambda_{0}=2 \pi f_{0} / c_{0}$. \\
\hline$n(\mathbf{r})$ & A refractive index distribution. \\
\hline$n(t)$ & A noise time series. \\
\hline$N_{\mathrm{f}}$ & $\begin{array}{l}\text { The number of focal points, however this is also used to indicate the number of time fre- } \\
\text { quency domain sample points. Be alert, the context will indicate what it represents. }\end{array}$ \\
\hline$N_{j}$ & The number of point scatterers. \\
\hline$N_{\text {rcv }}$ & The number of receivers. \\
\hline$N_{\text {src }}$ & The number of sources. \\
\hline$N_{t}$ & The number of time sample points. \\
\hline$N_{\mathrm{xvr}}$ & The number of transceivers. \\
\hline$O(\mathbf{r})$ & $\begin{array}{l}\text { The scattering object distribution function. Generally this is the unknown object to be } \\
\text { determined by the measurement system. }\end{array}$ \\
\hline$O_{b}(\mathbf{r})$ & $\begin{array}{l}\text { The scattering background distribution function. Generally this is known and considered } \\
\text { to be clutter. }\end{array}$ \\
\hline$\hat{o}_{\mathrm{mi}}$ & The migrated reconstruction of an object or object and background. \\
\hline$p(t)$ & The incident pulse. \\
\hline$p(\mathbf{r}, t)$ & $\begin{array}{l}\text { The incident pulse distribution. Generally, the point source assumption } p(\mathbf{r}, t)=p(t) \delta(\mathbf{r}- \\
\left.\mathbf{r}^{\prime}\right) \text { is made. }\end{array}$ \\
\hline$p\left(\mathbf{r}, \mathbf{R}_{n}^{\mathrm{src}}, t\right)$ & $\begin{array}{l}\text { The incident pulse distribution from a source located at } \mathbf{R}_{n}^{\mathrm{src}} . \text { Generally, the point source } \\
\text { assumption } p\left(\mathbf{r}, \mathbf{R}_{n}^{\mathrm{src}}, t\right)=p\left(\mathbf{R}_{n}^{\mathrm{src}}, t\right) \delta\left(\mathbf{r}-\mathbf{r}^{\prime}\right) \text { is made. }\end{array}$ \\
\hline & Continued ... \\
\hline
\end{tabular}




\begin{tabular}{|c|c|}
\hline \multicolumn{2}{|r|}{ A Variable Bestiary Continued } \\
\hline $\mathbf{r}_{\mathrm{f}}$ & The location of a focal point. In Cartesian coordinates, $\mathbf{r}_{\mathrm{f}}=\left(x_{f}, y_{f}, z_{f}\right)$. \\
\hline $\mathbf{R}_{m}^{\mathrm{rcv}}$ & $\begin{array}{l}\text { The location of the } m \text {-th receiver. A collection of receivers is identified by }\left\{\mathbf{R}_{m}^{\mathrm{rcv}}\right\}_{m=1}^{N_{\text {rcv }}} \text {. } \\
\text { A single receiver is indicated by omitting the subscript, } \mathbf{R}^{\mathrm{rcv}} \text {. In Cartesian coordinates, } \\
\mathbf{R}_{m}^{\mathrm{rcv}}=\left(x_{m}, y_{m}, z_{m}\right) \text {. }\end{array}$ \\
\hline $\mathbf{R}_{n}^{\mathrm{src}}$ & $\begin{array}{l}\text { The location of the } n \text {-th source. A collection of sources is identified by }\left\{\mathbf{R}_{n}^{\mathrm{src}}\right\}_{n=1}^{N_{\text {src }}} \text {. A } \\
\text { single source is indicated by omitting the subscript, } \mathbf{R}^{\mathrm{src}} \text {. In Cartesian coordinates, } \mathbf{R}_{n}^{\mathrm{src}}= \\
\left(x_{n}, y_{n}, z_{n}\right) \text {. }\end{array}$ \\
\hline $\mathbf{R}_{n}^{\mathrm{xvr}}$ & $\begin{array}{l}\text { The location of the } n \text {-th transducer. Generally used in multimonostatic systems when } \\
\mathbf{R}_{n}^{\mathrm{src}} \equiv \mathbf{R}_{n}^{\mathrm{rcv}} \text {. A collection of transceivers is indicated by }\left\{\mathbf{R}_{n}^{\mathrm{xvr}}\right\}_{n=1}^{N_{\mathrm{xvr}}} \text {. A single transceiver } \\
\text { is indicated by omitting the subscript, } \mathbf{R}^{\mathrm{xvr}} \text {. In Cartesian coordinates, } \mathbf{R}_{n}^{\mathrm{xvr}}=\left(x_{n}, y_{n}, z_{n}\right) \text {. }\end{array}$ \\
\hline $\mathbf{S}_{m}^{\mathrm{rcv}}$ & The $m$-th receiver's heading unit vector. \\
\hline $\mathbf{S}_{n}^{\mathrm{src}}$ & eading unit vector. \\
\hline $\mathbf{S}_{n}^{\mathrm{xvr}}$ & h transceiver's heading unit vector. \\
\hline & The time series origin. \\
\hline$t_{m f n}$ & $\begin{array}{l}\text { The time delay from the } n \text {-th source to a focal point, and from the focal point to the } m \text {-th } \\
\text { receiver: }\left(\left|\mathbf{R}_{m}^{\mathrm{rcv}}-\mathbf{r}_{\mathrm{f}}\right|+\left|\mathbf{r}_{\mathrm{f}}-\mathbf{R}_{n}^{\mathrm{src}}\right|\right) / c_{0} \text {. }\end{array}$ \\
\hline$t_{\mathrm{pe}}$ & Time at which the incident pulse achieves its peak magnitude value. \\
\hline & The \\
\hline$T_{n}^{\mathrm{src}}(\mathbf{r}, t)$ & The response characteristics of the $n$-th source \\
\hline $\mathbf{X}_{j}$ & $\begin{array}{l}\text { The location of the } j \text {-th point scatterer. A collection of point scatterers is indicated by } \\
\left\{\mathbf{X}_{j}\right\}_{j=1}^{N_{j}} \text {. When only one scatterer is present, the subscript is dropped and its location is } \\
\text { indicated by } \mathbf{X} \text {. }\end{array}$ \\
\hline
\end{tabular}




\section{Migration in a Nutshell}

The anatomy of a wave-based measurement system is presented in Figure 1. An excitement voltage, $e_{n}(t)$ drives the $n$-th source transducer with system response $T_{n}^{\mathrm{src}}(\mathbf{r}, t)$. Identical driving voltages may be applied to all the source transducers but the possibility of distinct sources is allowed, hence the $n$ subscript. The transducer converts the excitement voltage to the incident pulse via

$$
p\left(\mathbf{r}, \mathbf{R}_{n}^{\mathrm{src}}, t\right)=\int_{0}^{T} d t^{\prime} T_{n}^{\mathrm{src}}\left(\mathbf{r}, t-t^{\prime}\right) e_{n}\left(t^{\prime}\right)=T_{n}^{\mathrm{src}}(\mathbf{r}, \cdot) * e_{n}(\cdot)
$$

where $*$ indicates temporal convolution. When there is minimal (or no) information on the spatial-temporal transducer characteristics, it is common and convenient to set $T_{n}^{\mathrm{src}}(\mathbf{r}, t) \equiv \delta\left(\mathbf{r}-\mathbf{R}_{n}^{\mathrm{src}}\right) \delta\left(t-t^{\prime}\right)$ and express the incident field as

$$
p\left(\mathbf{r}, \mathbf{R}_{n}^{\mathrm{src}}, t\right)=e_{n}(t) \delta\left(\mathbf{r}-\mathbf{R}_{n}^{\mathrm{src}}\right) .
$$

Assuming identical excitement voltages are applied to all the sources, the incident pulse expression is

$$
p\left(\mathbf{r}, \mathbf{R}_{n}^{\mathrm{src}}, t\right)=p(t) \delta\left(\mathbf{r}-\mathbf{R}_{n}^{\mathrm{src}}\right) .
$$

The source transducer emits the incident field. It is shown in Section 4 the incident field is related to the incident pulse via

$$
\psi^{\mathrm{inc}}\left(\mathbf{r}, \mathbf{R}_{n}^{\mathrm{src}}, t\right)=\int_{0}^{T} d t^{\prime} G_{0}\left(\mathbf{r}-\mathbf{R}_{n}^{\mathrm{src}}, t-t^{\prime}\right) p\left(t^{\prime}\right)=G_{0}\left(\mathbf{r}-\mathbf{R}_{n}^{\mathrm{src}}, \cdot\right) * p(\cdot)
$$

where $G_{0}(\mathbf{r}, t)$ is the background Green function which describes how the incident field propagates in the environment. Generally, the zero subscript indicates the free space Green function. A different subscript will be used with a known but inhomogeneous background. For example the Green function for a two layer medium is indicated by $G_{1}(\mathbf{r}, t)$. The field interacts with the scattering object, $O(\mathbf{r})$, presumed to be unknown (and to be determined), and possibly a known background, $O_{b}(\mathbf{r})$. The result of the interaction is the scattered field, $\psi^{\text {scat }}\left(\mathbf{r}, \mathbf{R}_{n}^{\mathrm{src}}, t\right)$ which propagates to and is measured by the $m$-th receiving transducer with system response $T_{m}^{\mathrm{rcv}}(\mathbf{r}, t)$. The scattered field originating from the known background is referred to as clutter. The measured voltage recorded by the transducer is $v_{m, n}^{\text {scat }}(t)$. This is the multistatic data matrix. The flow diagram models a fully multistatic system but can represent a multimonostatic system when $m \equiv n$. In this case the measured data are known as the multimonostatic data matrix. The various measurement systems are defined in Appendix B. The flow diagram can model both reflection and transmission mode. In the latter case, the background object, $O_{b}(\mathbf{r})$, is replaced by $O_{b}(\mathbf{r})+1$ and scattered field, $\psi^{\text {scat }}\left(\mathbf{r}, \mathbf{R}_{n}^{\text {src }}, t\right)$, by the total field,

$$
\psi^{\mathrm{tot}}\left(\mathbf{r}, \mathbf{R}_{n}^{\mathrm{src}}, t\right) \equiv \psi^{\mathrm{inc}}\left(\mathbf{r}, \mathbf{R}_{n}^{\mathrm{src}}, t\right)+\psi^{\mathrm{scat}}\left(\mathbf{r}, \mathbf{R}_{n}^{\mathrm{src}}, t\right)
$$

to account for the incident field.

For example, an expression for the scattered field resulting from a collection of point scatterers with locations $\left\{\mathbf{X}_{j}\right\}_{j=1}^{J}$ is derived in Section 6.2:

$$
\psi_{B}^{\mathrm{scat}}\left(\mathbf{r}, \mathbf{R}_{n}^{\mathrm{xvr}}, t\right)=-\frac{1}{(4 \pi)^{2} c_{0}^{2}} \sum_{j=1}^{J} \frac{\tau_{j}}{\left|\mathbf{r}-\mathbf{X}_{j}\right| \mid \mathbf{X}_{j}-\mathbf{R}_{n}^{\mathrm{xvr} \mid}} p^{\prime \prime}\left(t-\frac{\left|\mathbf{r}-\mathbf{X}_{j}\right|+\left|\mathbf{X}_{j}-\mathbf{R}_{n}^{\mathrm{xvr}}\right|}{c_{0}}\right)
$$

where the double prime on $p(\cdot)$ indicates the second derivative with respect to its argument, $\tau_{j}$ is the scattering amplitude, and, for precision, a $B$ subscript indicates the field was derived under the Born approximation [6] which neglects multiple, inter-scatterer scattering. For a single point scatter, $J \equiv 1$, the expression reduces to an exact formulation,

$$
\psi^{\mathrm{scat}}\left(\mathbf{r}, \mathbf{R}_{n}^{\mathrm{xvr}}, t\right)=-\frac{1}{(4 \pi)^{2} c_{0}^{2}} \frac{\tau}{|\mathbf{r}-\mathbf{X}|\left|\mathbf{X}-\mathbf{R}_{n}^{\mathrm{xvr}}\right|} p^{\prime \prime}\left(t-\frac{|\mathbf{r}-\mathbf{X}|+\left|\mathbf{X}-\mathbf{R}_{n}^{\mathrm{xvr}}\right|}{c_{0}}\right) .
$$


When the scattered field is evaluated at a transceiver, the expression becomes

$$
\psi^{\mathrm{scat}}\left(\mathbf{R}_{n}^{\mathrm{xvr}}, \mathbf{R}_{n}^{\mathrm{xvr}}, t\right)=-\frac{1}{(4 \pi)^{2} c_{0}^{2}} \frac{\tau}{\left|\mathbf{X}-\mathbf{R}_{n}^{\mathrm{xvr}}\right|^{2}} p^{\prime \prime}\left(t-\frac{2\left|\mathbf{X}-\mathbf{R}_{n}^{\mathrm{xvr}}\right|}{c_{0}}\right) .
$$

Convolving the scattered field with the transducer characteristic function results in the received voltage signal,

$$
s_{n}(t)=\int_{0}^{T} d t^{\prime} T_{n}^{\mathrm{xvr}}\left(\mathbf{r}, t-t^{\prime}\right) \psi^{\mathrm{scat}}\left(\mathbf{R}_{n}^{\mathrm{xvr}}, \mathbf{R}_{n}^{\mathrm{xvr}}, t^{\prime}\right)=T_{n}^{\mathrm{xvr}}(\mathbf{r}, \cdot) * \psi^{\mathrm{scat}}\left(\mathbf{R}_{n}^{\mathrm{xvr}}, \mathbf{R}_{n}^{\mathrm{xvr}}, \cdot\right) .
$$

The multi(mono)static data matrix is the received voltage signal with additive system noise,

$$
v_{n}^{\mathrm{scat}}(t)=s_{n}(t)+n(t) .
$$

For this example, the transducer characteristic function is taken to be one, and the noise time series is ignored. The result is

$$
v_{n}^{\mathrm{scat}}(t) \equiv \psi^{\mathrm{scat}}\left(\mathbf{R}_{n}^{\mathrm{xvr}}, \mathbf{R}_{n}^{\mathrm{xvr}}, t\right)
$$

As a simple simulated scattering example, consider the incident pulse time series, $p(t)$, presented in Figure 2: four cycles of a $20 \mathrm{kHz}$ Gaussian windowed sinusoid:

$$
p(t)=\sin \left(\omega_{0} t\right) \exp \left(-\frac{1}{2}\left(\frac{\omega_{0} t}{N_{\mathrm{cyc}}}\right)^{2}\right)
$$

where $\omega_{0}=2 \pi \times 20 \times 10^{3}$, and $N_{\text {cyc }}=4$. In all the examples, the spatial coordinates are scaled to the wavelength of the peak, $20 \mathrm{kHz}$, frequency, $\lambda_{0}=1.715$ centimeters with a wave propagation velocity of $c_{0}=343 \mathrm{~meters} / \mathrm{second}$ (the speed of sound in air). Figure 3 shows the geometry of the simulated examples. The transceiver array is located along the $y \equiv 0$ axis with coordinates, $\left\{\mathbf{R}_{n}^{\mathrm{xvr}}\right\}_{n=1}^{N_{\mathrm{xvr}}}$ where $N_{\mathrm{xvr}}$ is the number of transceivers. Point scatterer locations are indicated by $\left\{\mathbf{X}_{j}\right\}_{j=1}^{N_{j}}$ where $N_{j}$ is the number of scatterers. When there is only one scatterer, the $j$ subscript is omitted. The scatterer(s) are located at a range of $10 \lambda_{0}$ from the array and their locations are indicated by circles of diameter $\lambda_{0}$.

Figures 4 through 6 present a sequence of "snapshots" of the incident and scattered field propagation for a single scatterer located at X. As derived in Eqn. (29) of Section 4, the the incident field propagation is described by

$$
\psi^{\mathrm{inc}}\left(\mathbf{r}, \mathbf{R}_{n}^{\mathrm{src}}, t\right)=\frac{1}{4 \pi\left|\mathbf{r}-\mathbf{R}_{n}^{\mathrm{src}}\right|} p\left(t-\frac{\left|\mathbf{r}-\mathbf{R}_{n}^{\mathrm{src}}\right|}{c_{0}}\right) .
$$

The time steps are expressed in terms of the number of wavelengths the incident field has propagated:

$$
t_{k}=\frac{\lambda_{0}}{c_{0}} k
$$

where $k=1,2,3, \cdots$. By time step $k \equiv 15$ (Figure 5), the incident field has interacted with the point scatterer and the scattered field has been created. By time step 27 (Figure 6), the scattered field has reached the transducer located at $\mathbf{R}_{n+1}^{\mathrm{xvr}}$. The vertical line descending from its location indicates the received measured field time series, $v_{n+1}^{\text {scat }}(t)$ and the leading edge of the pulse has manifested itself. The scattered pulse arrives after a delay of $2\left|\mathbf{X}-\mathbf{R}_{n}^{\mathrm{xvr}}\right| / c_{0}$. The time shift (delay) in the scattered field of Eqn. (8) and its corresponding measured voltage of Eqn. (11) depends upon the distance between the transceiver and the point scatterer,

$$
\left|\mathbf{X}-\mathbf{R}_{n}^{\mathrm{xvr}}\right| \equiv \sqrt{\left(x_{s}-x_{n}\right)^{2}+\left(y_{s}-y_{n}\right)^{2}+\left(z_{s}-z_{n}\right)^{2}}
$$

where $\mathbf{X}=\left(x_{s}, y_{s}, z_{s}\right)$ and $\mathbf{R}_{n}^{\mathrm{xvr}}=\left(x_{n}, y_{n}, z_{n}\right)$. As the transceiver progresses from one location to the next acquiring data, two important concepts arise from the spatial dependence of the time shift: 
- The moveout $[3,4,5]$, known colloquially as the "hyperbolic smile", arises when Eqn. (15) is interpreted as a function of transceiver location, $\mathbf{R}_{n}^{\mathrm{xvr}}$ with fixed scatterer location, $\mathbf{X}$,

$$
\left\{\left|\mathbf{X}-\mathbf{R}_{n}^{\mathrm{xvr}}\right|\right\}_{n=1}^{N_{\mathrm{xvr}}} .
$$

The locus of points described by Eqn. (16) trace the "smile".

- The ambiguity curve, results from interpreting Eqn. (15) as a constant-valued function of scatterer location, $\mathbf{X}$, with fixed transceiver location, $\mathbf{R}_{n}^{\mathrm{xvr}}$,

$$
\left|\mathbf{r}-\mathbf{R}_{n}^{\mathrm{xvr}}\right|=\underbrace{\left|\mathbf{X}-\mathbf{R}_{n}^{\mathrm{xvr}}\right|}_{\text {constant }} .
$$

Scatterers located on this curve will all yield identical measured fields when seen at a receiver co-located with the source. The ambiguity arises from the delay in the return is a function only of the difference between the transceiver and scatterer and not the scattering angle. Single scatterers located at identical ranges yield identical scattered fields at the transceiver.

These concepts are demonstrated in Figure 7. The location ambiguity is resolved by combining the measured fields from multiple spatially diverse (separated) transceivers. One method of combining the measured fields is migration. Superposing the range ambiguity curve for each transceiver, the location of the returned shifted and scaled pule is traced back to the actual scatterer location. Migration focuses or maps the smiles to scattering points.

Migration, in a nutshell, consists of undoing the forward scattering and propagation effects on the collection of measured voltages, $\left\{v_{n}^{\text {scat }}(t)\right\}_{n=1}^{N_{\text {xvr }}}$, to obtain the scatter location and amplitude. These effects are

- A time shift of the pulse (technically, the second derivative of the pulse) given by $\frac{2\left|\mathbf{X}-\mathbf{R}_{n}^{\mathrm{xvr}}\right|}{c_{0}}$,

- And a range scaling of $\frac{1}{\left|\mathbf{X}-\mathbf{R}_{n}^{\mathrm{xvr}}\right|^{2}}$.

Assuming the scatterer location is unknown (certainly, if its location were known, there would be no need to employ the measurement system of Figure 1 other than as an exercise), a guess at its location is made, a migration performed, and the result analyzed to infer the (non-)existence of any scatter at the guessed location. The guessed location is referred to as the focal point and it is indicated mathematically by $\mathbf{r}_{\mathrm{f}}$.

Most generally, a collection of focal points, $\left\{\mathbf{r}_{\mathrm{f}, n}\right\}_{n=1}^{N_{\mathrm{f}}}$, is selected over the volume being interrogated. Migrations are performed to each focal point and an "image" of the volume created. High values or "hot spots" in the image indicate the location and scattering amplitude of a scatterer. Mathematically, the combined migrations are described as

$$
\hat{o}_{\mathrm{mig}}\left(\mathbf{r}_{\mathrm{f}}\right)=\frac{1}{N_{\mathrm{xvr}}} \sum_{n=1}^{N_{\mathrm{xvr}}}\left|\mathbf{r}_{\mathrm{f}}-\mathbf{R}_{n}^{\mathrm{xvr}}\right|^{2} v_{n}^{\text {scat }}\left(t_{\text {peak }}+\frac{2\left|\mathbf{r}_{\mathrm{f}}-\mathbf{R}_{n}^{\mathrm{xvr}}\right|}{c_{0}}\right),
$$

where $\hat{o}_{\text {mig }}\left(\mathbf{r}_{\mathrm{f}}\right)$ is an estimate of the scattering object achieved through migration. Substituting Eqn. (8),

$$
\hat{o}_{\text {mig }}\left(\mathbf{r}_{\mathrm{f}}\right)=-\frac{\tau}{(4 \pi)^{2} c_{0}^{2} N_{\mathrm{xvr}}} \sum_{n=1}^{N_{\mathrm{xvr}}} \frac{\left|\mathbf{r}_{\mathrm{f}}-\mathbf{R}_{n}^{\mathrm{xvr}}\right|^{2}}{\left|\mathbf{X}-\mathbf{R}_{n}^{\mathrm{xvr}}\right|^{2}} p^{\prime \prime}\left(t_{\text {peak }}-\frac{2\left|\mathbf{X}-\mathbf{R}_{n}^{\mathrm{xvr}}\right|}{c_{0}}+\frac{2\left|\mathbf{r}_{\mathrm{f}}-\mathbf{R}_{n}^{\mathrm{xvr}}\right|}{c_{0}}\right),
$$

it is observed when a focal point coincides with the scatterer location, $\mathbf{r}_{\mathrm{f}} \equiv \mathbf{X}$, the value of the migration reduces to the coherent sum of the pulse derivative evaluated at the pulse peak time:

$$
\hat{o}_{\mathrm{mig}}(\mathbf{X})=-\frac{\tau}{(4 \pi)^{2} c_{0}^{2}} p^{\prime \prime}\left(t_{\text {peak }}\right) .
$$

For $\mathbf{r}_{\mathrm{f}} \neq \mathbf{X}$, two cases must be considered, 


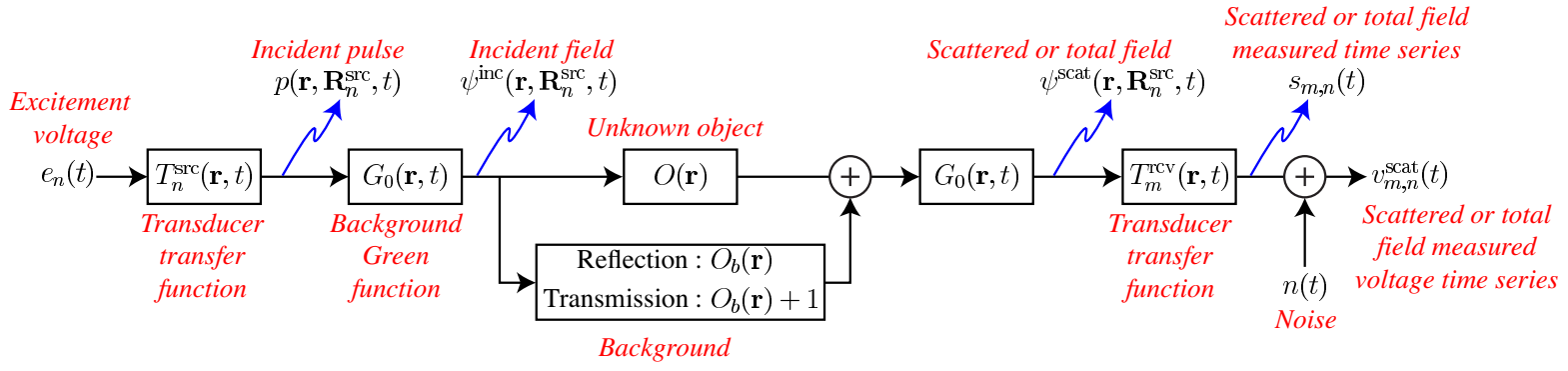

Figure 1: Anatomy of a wave-based measurement system. An excitement voltage, $e_{n}(t)$ drives the $n$-th source transducer with system response $T_{n}^{\mathrm{src}}(\mathbf{r}, t)$ producing the incident pulse, $p\left(\mathbf{r}, \mathbf{R}_{n}^{\mathrm{src}}, t\right)$. The emitted incident field, $\psi^{\text {inc }}\left(\mathbf{r}, \mathbf{R}_{n}^{\mathrm{src}}, t\right)$, propagates into the environment as indicated by the Green function $G_{0}(\mathbf{r}, t)$. The field interacts with the scattering object, $O(\mathbf{r})$, presumed to be unknown, and possibly a known background, $O_{b}(\mathbf{r})$. The result of the interaction is the scattered field, $\psi^{\mathrm{scat}}\left(\mathbf{r}, \mathbf{R}_{n}^{\mathrm{src}}, t\right)$ which propagates to and is measured by the $m$-th receiving transducer with system response $T_{m}^{\mathrm{rcv}}(\mathbf{r}, t)$. The measured voltage recorded from the transducer is $v_{m, n}^{\mathrm{scat}}(t)$. This is the multistatic data matrix. The flow diagram models a fully multistatic system but can represent a multimonostatic system when $m \equiv n$. It can model both reflection and transmission mode. In the latter case, we replace $O_{b}(\mathbf{r})$ by $O_{b}(\mathbf{r})+1$ and $\psi^{\mathrm{scat}}\left(\mathbf{r}, \mathbf{R}_{n}^{\mathrm{src}}, t\right)$ by $\psi^{\text {tot }}\left(\mathbf{r}, \mathbf{R}_{n}^{\mathrm{src}}, t\right)$ to account for the incident field.

1. When the shifts exceed the pulse length ${ }^{1}$,

$$
p^{\prime \prime}\left(t_{\text {peak }}-\frac{2\left|\mathbf{X}-\mathbf{R}_{n}^{\mathrm{xvr}}\right|}{c_{0}}+\frac{2\left|\mathbf{r}_{\mathrm{f}}-\mathbf{R}_{n}^{\mathrm{xvr}}\right|}{c_{0}}\right)=0
$$

and the sum of Eqn. (19) is zero.

2. When the shifts are less than the pulse length, the sum of Eqn. (19) will result in patterns of coherent interference, that is regions of constructive and destructive interference depending upon the shift.

Examples of delay, scale, and sum migration are demonstrated in Figures 8 and 9 for two focal points:

- The first focal point coincides with the scatterer location, $\mathbf{r}_{\mathrm{f}} \equiv \mathbf{X}$;

- The second focal point does not coincide with the scatterer location, $\mathbf{r}_{\mathrm{f}} \neq \mathbf{X}$.

The top plot of Figure 8 presents the multimonostatic data matrix of Figure 7 overlaid on the migrated image for reference. The two bottom plots demonstrate the concept of migration in terms of focusing of the measured time series. For each of the focal points, the range scaling of $\left|\mathbf{r}_{\mathrm{f}}-\mathbf{R}_{n}^{\mathrm{xvr}}\right|^{-2}$ is applied to the time series. Each time series is rotated about the transducer location along its ambiguity curve until the trace intersects the focal point where the series are summed. The scaled and rotated time series constructively interfere at $\mathbf{r}_{\mathrm{f}} \equiv \mathbf{X}$, whereas there is minimal contribution when $\mathbf{r}_{\mathrm{f}} \neq \mathbf{X}$ resulting from the non-uniform scaling and phase of the returns along the ambiguity curve.

Figure 9 presents the delay, scale, and summation steps for each of the focal points. The top plots show the time series after they have been shifted by $\left|\mathbf{r}_{\mathrm{f}}-\mathbf{R}_{n}^{\mathrm{xvr}}\right| / c_{0}$. When $\mathbf{r}_{\mathrm{f}} \equiv \mathbf{X}$, the phases of the time series align whereas they do not when $\mathbf{r}_{\mathrm{f}} \neq \mathbf{X}$. In fact, in some cases the scattered pulse shifts out of the time series. The bottom plots present the delayed time series scaled by $\left|\mathbf{r}_{\mathrm{f}}-\mathbf{R}_{n}^{\mathrm{xvr}}\right|^{-2}$. The time series for $\mathbf{r}_{\mathrm{f}} \equiv \mathbf{X}$ are uniformly scaled.

Finally, the time series are summed over all transceivers as in Eqn. (18). The resulting sums are plotted in Figure 10. The value of the migration is the time series evaluated at the range of the focal point. These are indicated in the plot.

\footnotetext{
${ }^{1}$ For the pulse of Eqn. (12), this is approximately $\frac{2 N_{\mathrm{cyc}}}{\omega_{0}}$
} 

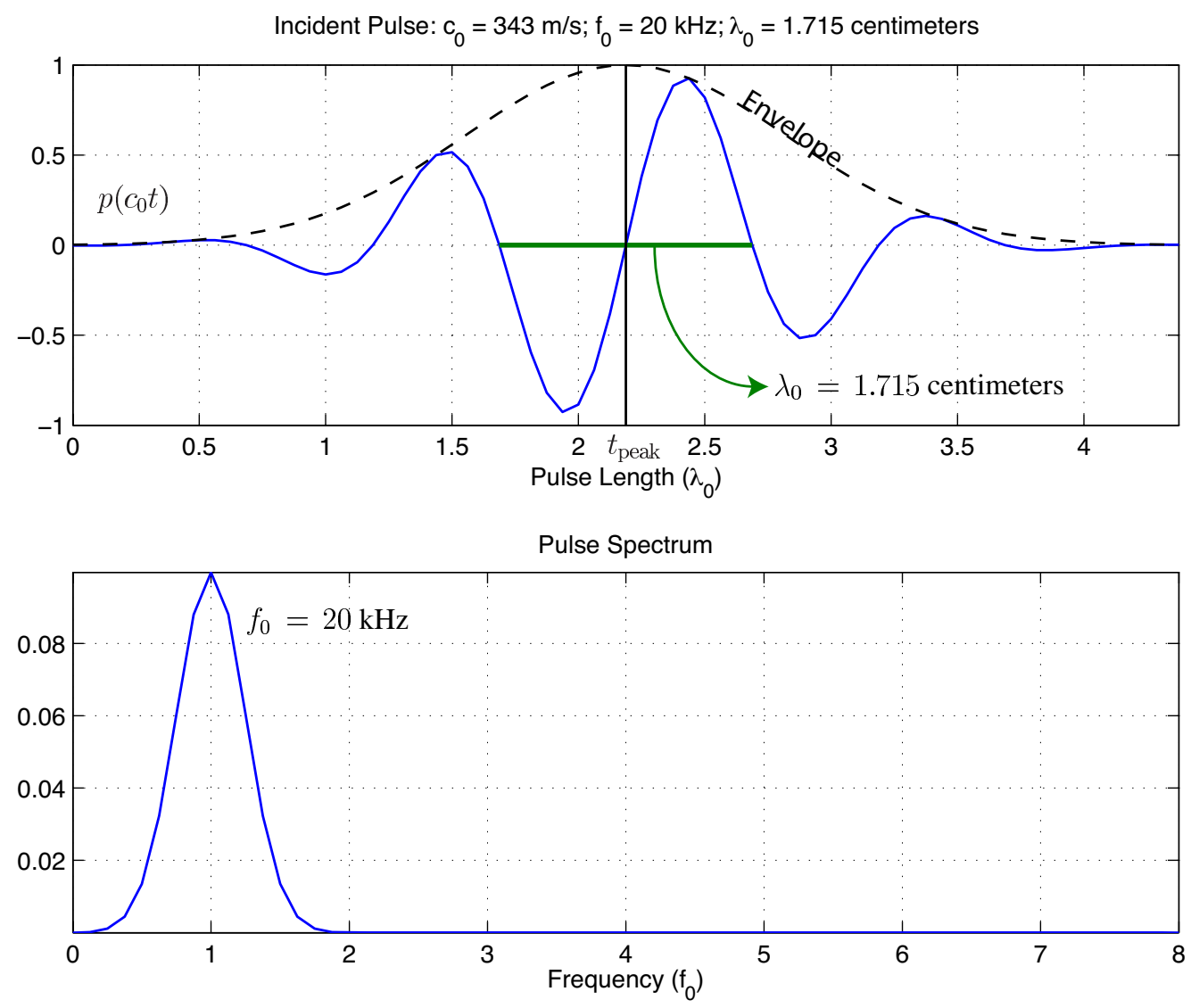

Figure 2: The examples used in the tutorial use a background velocity of $343 \mathrm{~m} / \mathrm{s}$ and a $20 \mathrm{kHz}$ Gaussian windowed sinusoid incident pulse, $p(t)$. All coordinates used in the figures are scaled to the pulse wavelength, $\lambda_{0}$, of $1.715 \mathrm{~cm}$. 


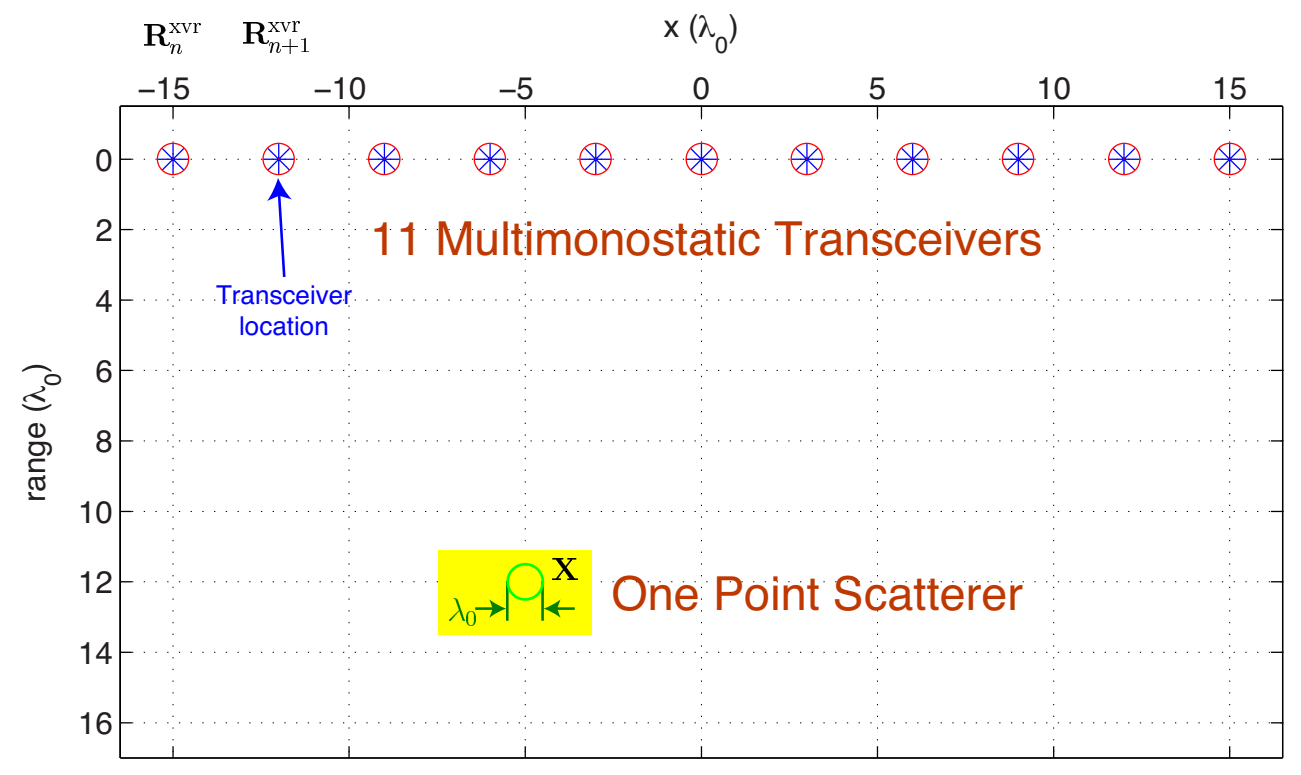

Figure 3: The geometry used in the simulated examples. The spatial dimensions are scaled to the pulse wavelength, $\lambda_{0}$, of $1.715 \mathrm{~cm}$. The transceiver array is located along the $y \equiv 0$ axis. The scatterer location(s) are indicated by circles of diameter $\lambda_{0}$.

\subsection{A Brief, Graphical Description of Resolution Limit}

Resolution limit (at times identified as the "classical" resolution limit) of an aperture or array is defined as the minimum distinguishable distance between two point scatterers. Mathematically it is derived as [6]:

$$
\Delta h=1.22 \frac{r \lambda_{0}}{A},
$$

where

$\Delta h$ is the spatial separation between the two point scatterers,

$r$ is the range (distance) from the array to the plane of the scatterers,

$\lambda_{0}$ is the wavelength,

$A$ is the aperture or array size.

A caveat: this is a single frequency definition whereas the problem studied here is wide band (see the incident pulse spectrum of Figure 2). The wavelength chosen to estimate the resolution limit is that of the peak, $20 \mathrm{kHz}$, frequency $(1.715 \mathrm{~cm}$ wavelength), however a more appropriate wavelength is that corresponding to the highest usable frequency within the band width of the pulse spectrum.

Consider the measurement system of Figure 11. Two point scatterers are placed at $r=10 \lambda_{0}$ from the array, separated by $\Delta h=\lambda_{0}$. The following configurations are studied graphically in Figure 12:

1. (a) A single transceiver. With no other information, the migrator can only "smear" the single return over the ambiguity curve.

2. (b) A two element array with aperture size $A=12.20 \lambda_{0}$. According to Eqn. (21), the scatterers are just resolved.

3. The cases presented in sub-figures (c) through (g) maintain the resolution limit aperture of $A=12.20 \lambda_{0}$ while increasing the transceiver population. They demonstrate increasing the number of elements increases the signalto-noise ratio and reduces artifact clutter. 

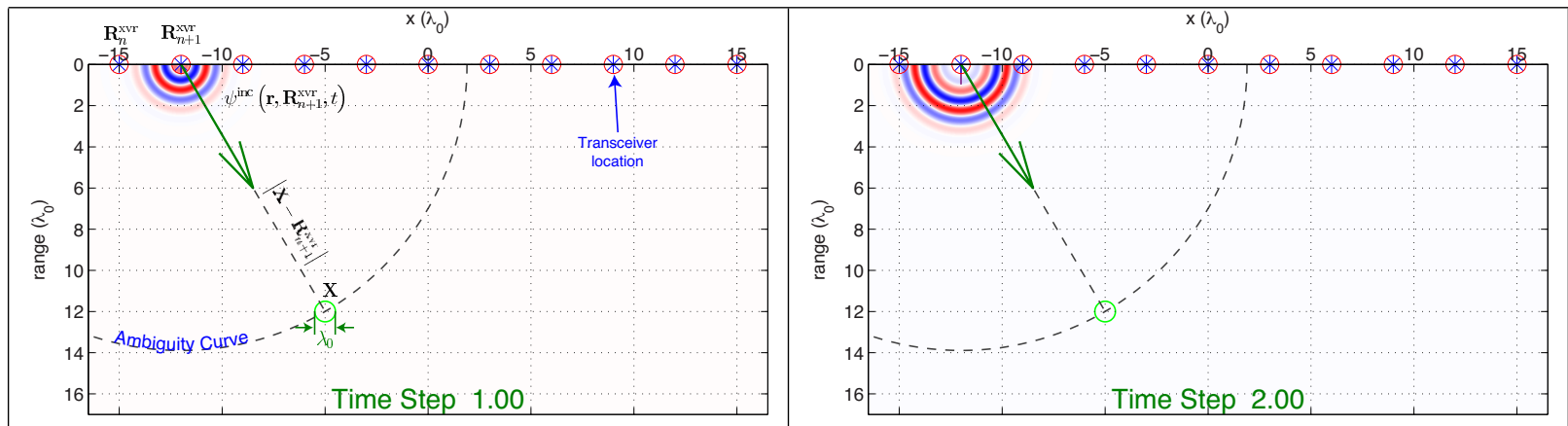

(a) Time step 1.

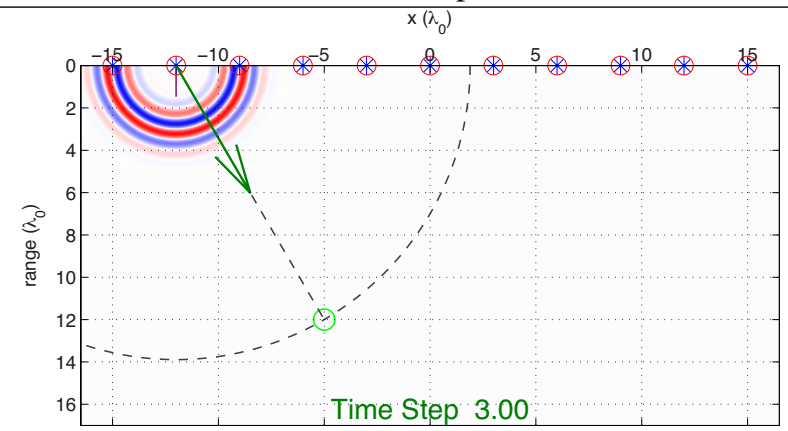

(b) Time step 2.

(c) Time step 3.

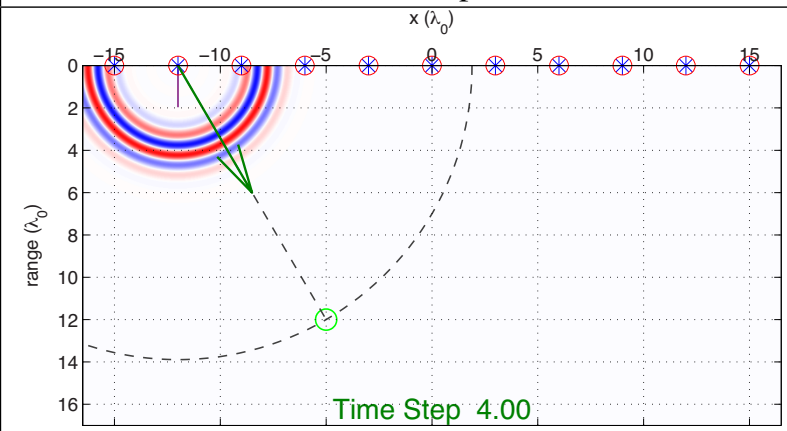

(d) Time step 4.

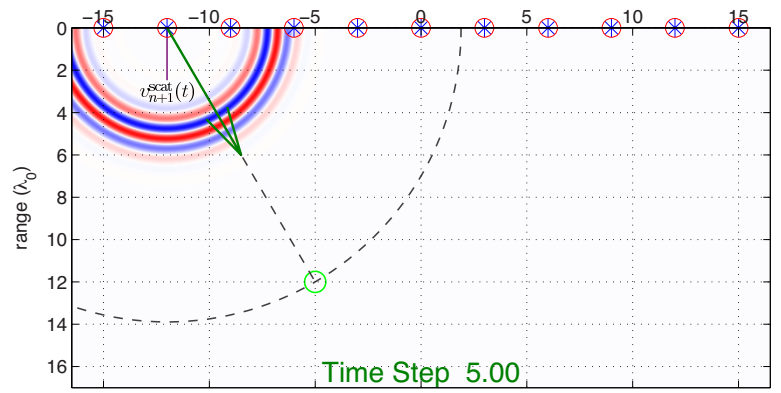

(e) Time step 5.

Figure 4: A sequence of "snapshots" of the incident and scattered field propagation. The "time steps" are in terms of wavelength: $t_{k}=k \lambda_{0} / c_{0}$ where $k=1,2,3,4,5$. 


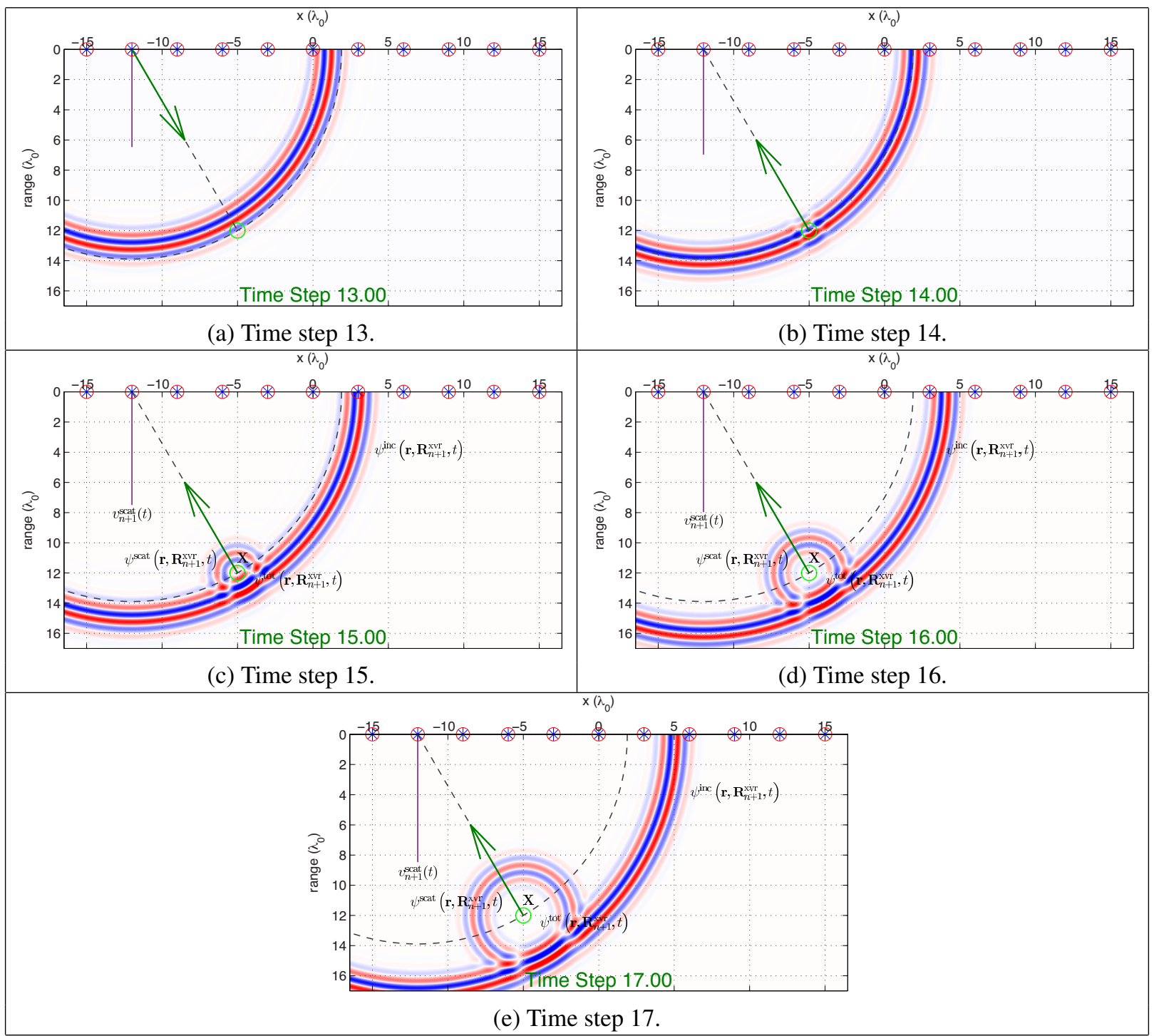

Figure 5: A sequence of "snapshots" of the incident and scattered field propagation. The "time steps" are in terms of wavelength: $t_{k}=k \lambda_{0} / c_{0}$ where $k=13,14,15,16,17$. 


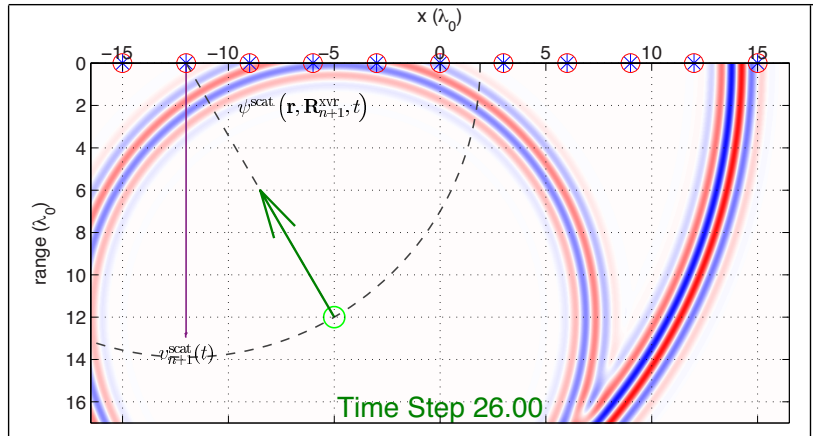

(a) Time step 26.

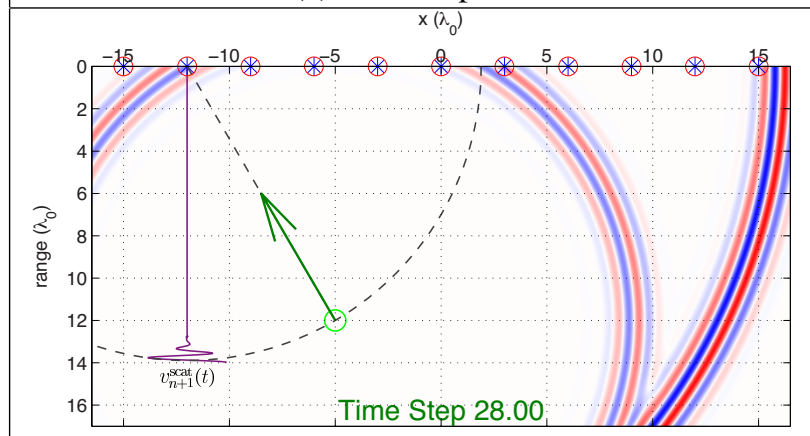

(c) Time step 28.

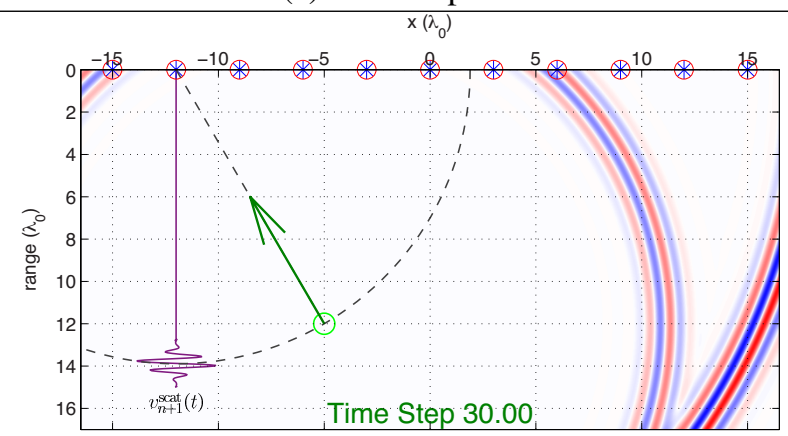

(e) Time step 30 .

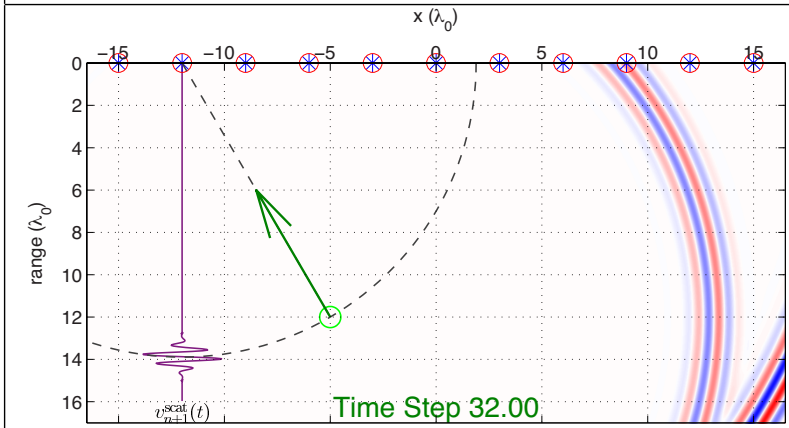

(g) Time step 32.

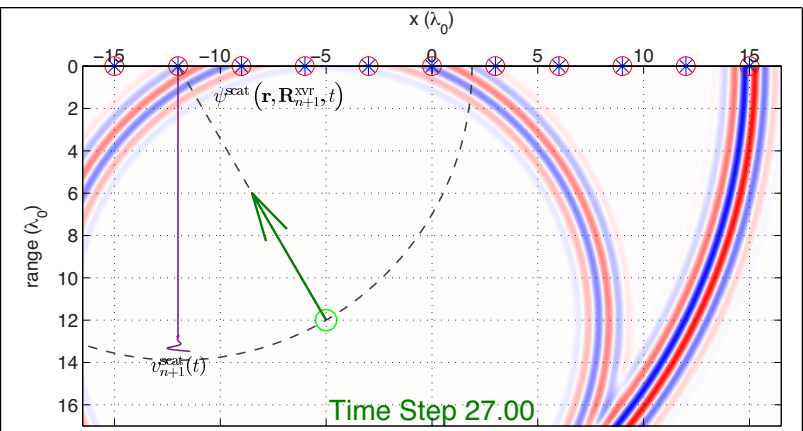

(b) Time step 27.

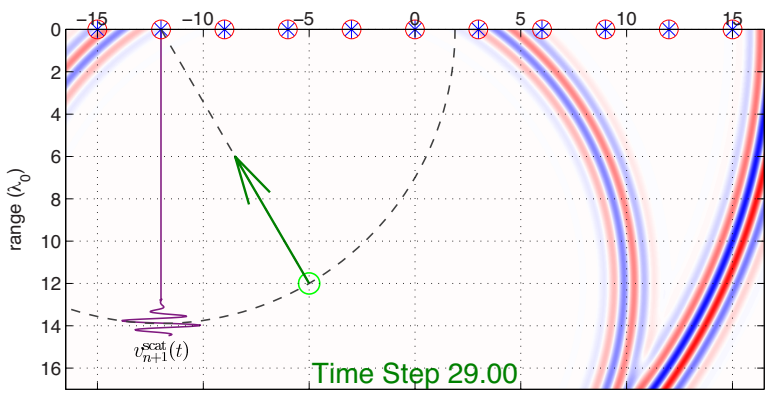

(d) Time step 29.

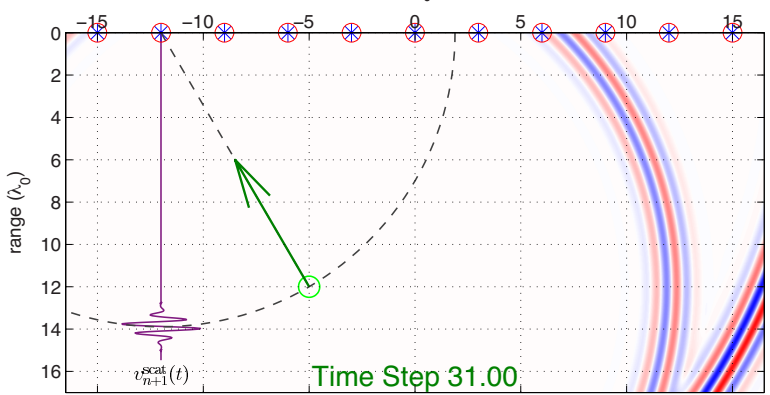

(f) Time step 31.

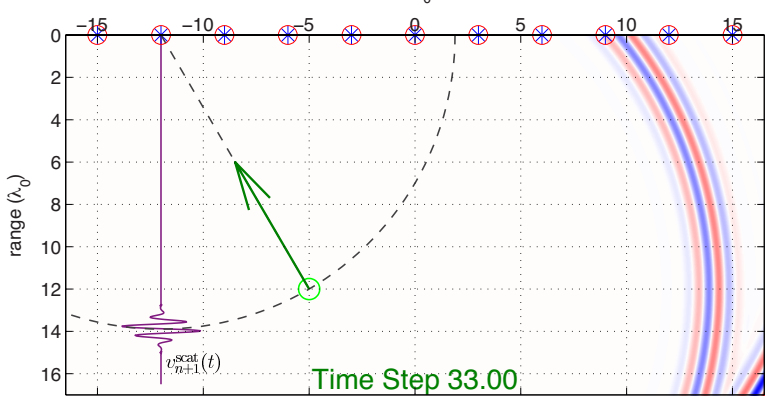

(h) Time step 33.

Figure 6: A sequence of "snapshots" of the incident and scattered field propagation. The "time steps" are in terms of wavelength: $t_{k}=k \lambda_{0} / c_{0}$ where $k=26,27,28,29,30,31,32,33$. 


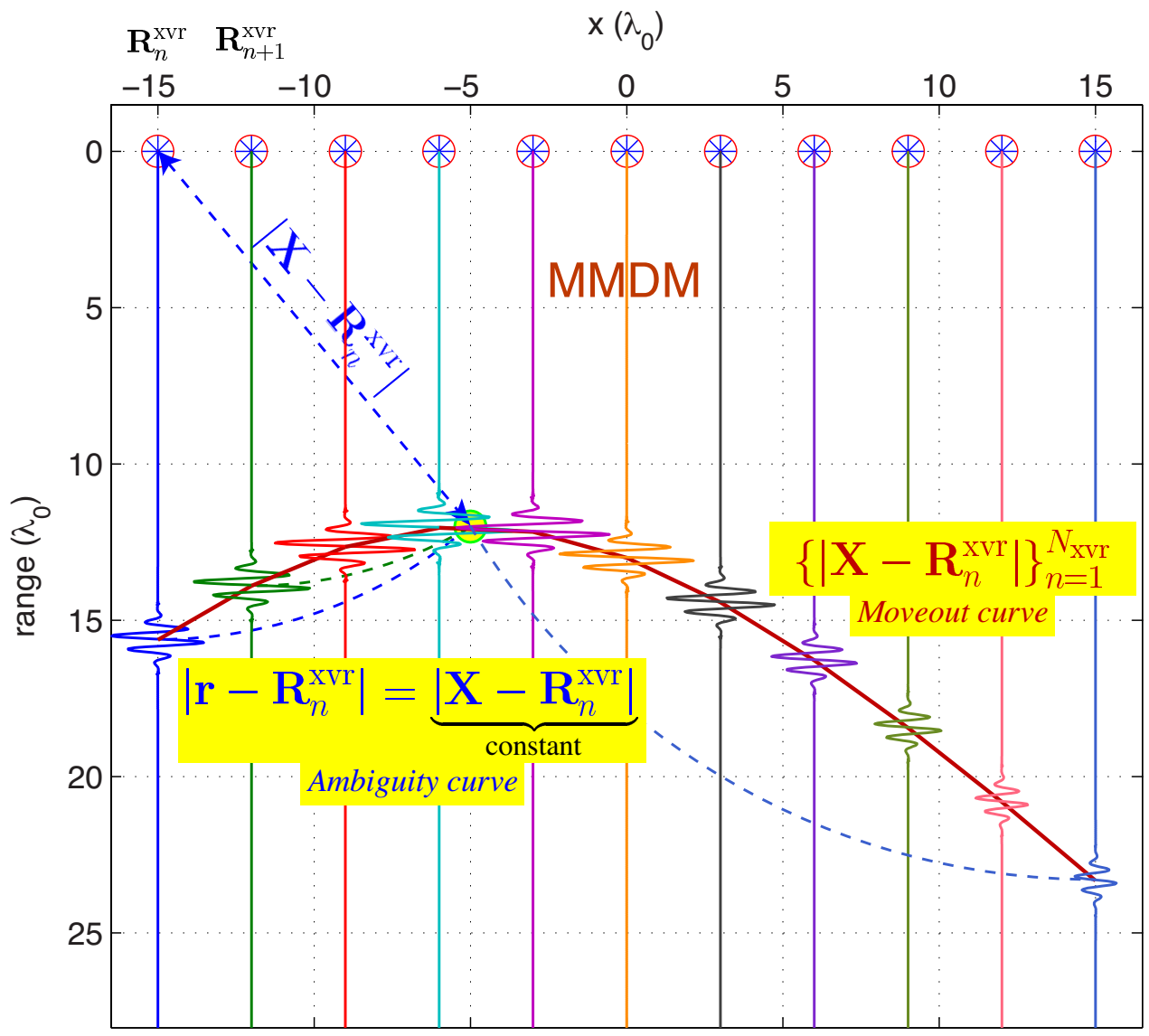

Figure 7: Visualization of the multimonostatic data matrix. The ambiguity curse results from the nature of the scattering which depends only upon the distance between the transceiver and the scatterer, and not the angle. The moveout curve is a consequence of the displacement of the transceivers. 


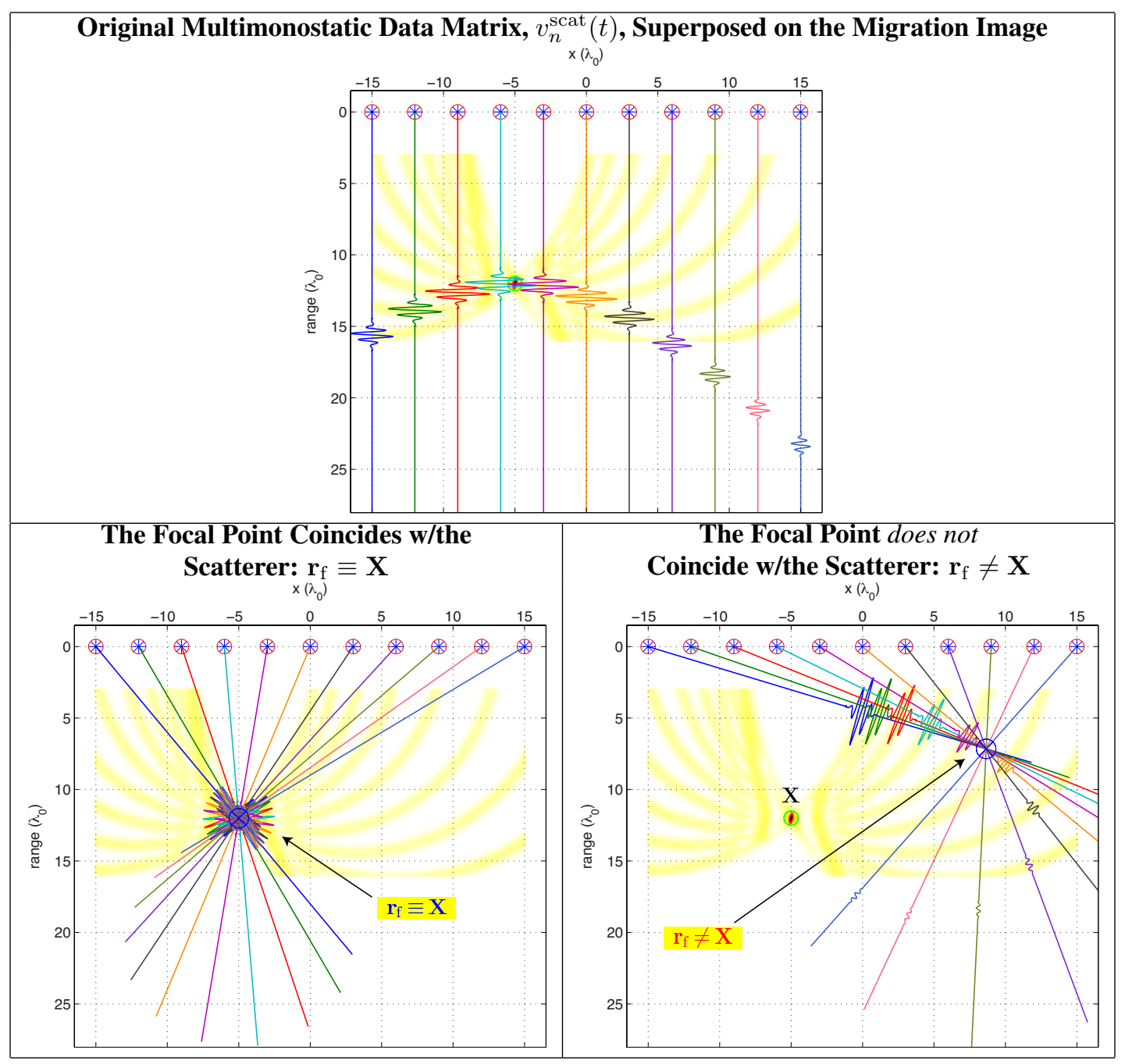

Figure 8: Focusing demonstration: the multimonostatic data matrix time series are scaled and rotated along their respective ambiguity curves to the focal point. A coherent sum is achieved when the focal point coincides with the scatterer location. 


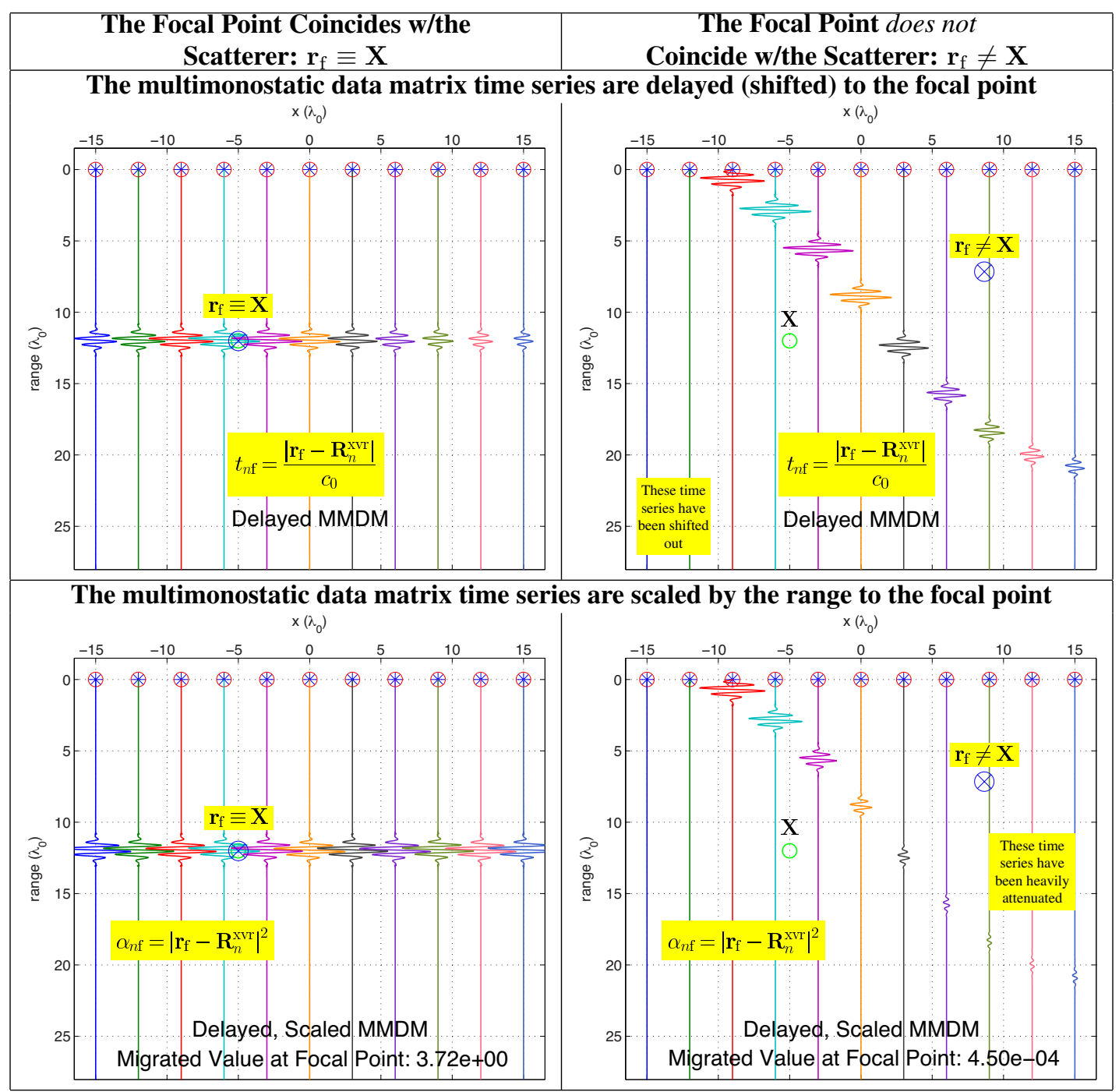

Figure 9: Migration steps. 


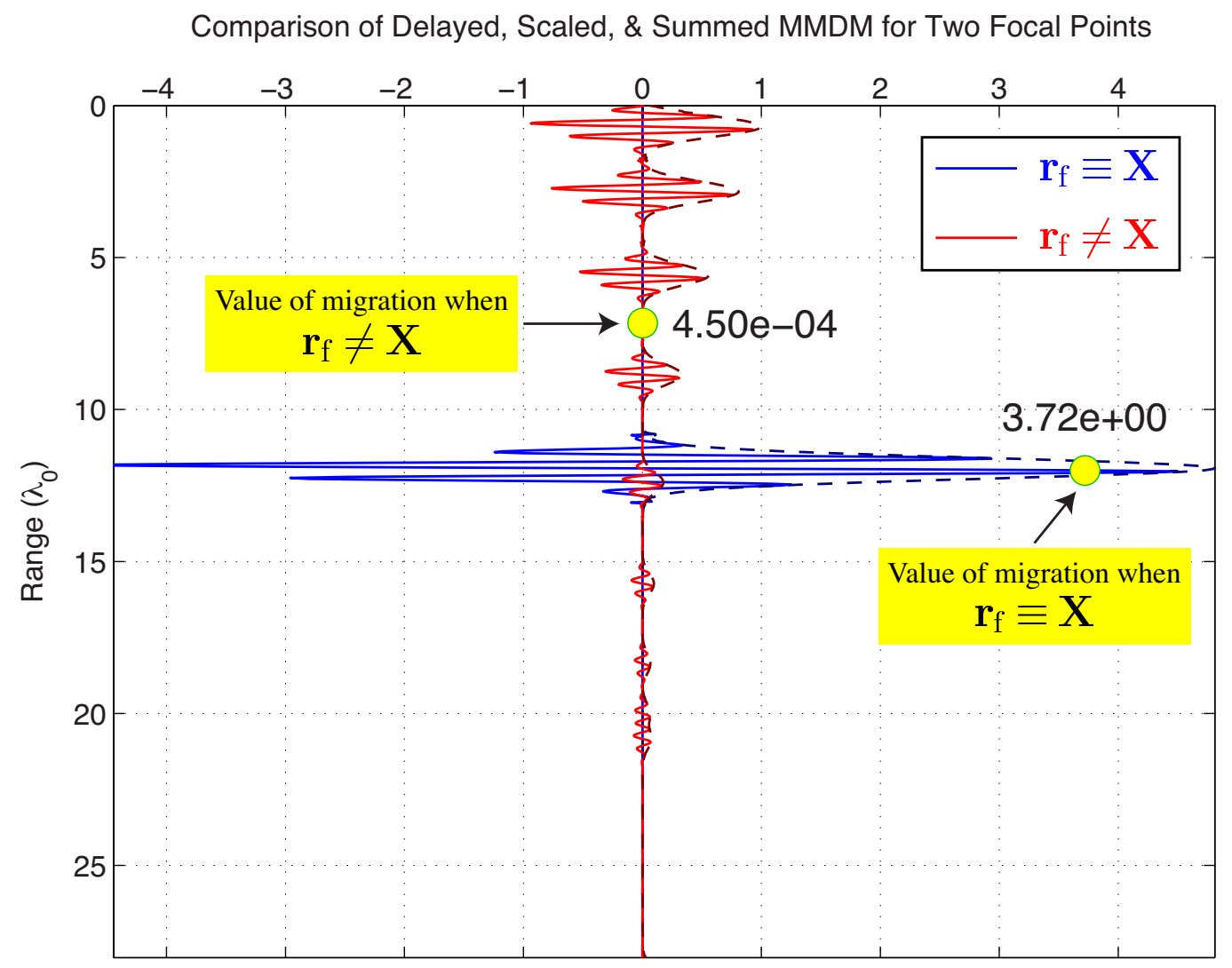

Figure 10: The delayed and scaled multimonostatic data matrix time series of Figure 9 are summed. The values of the sums at the focal points are shown. A coherent sum is achieved when the focal point coincides with the scatterer. 


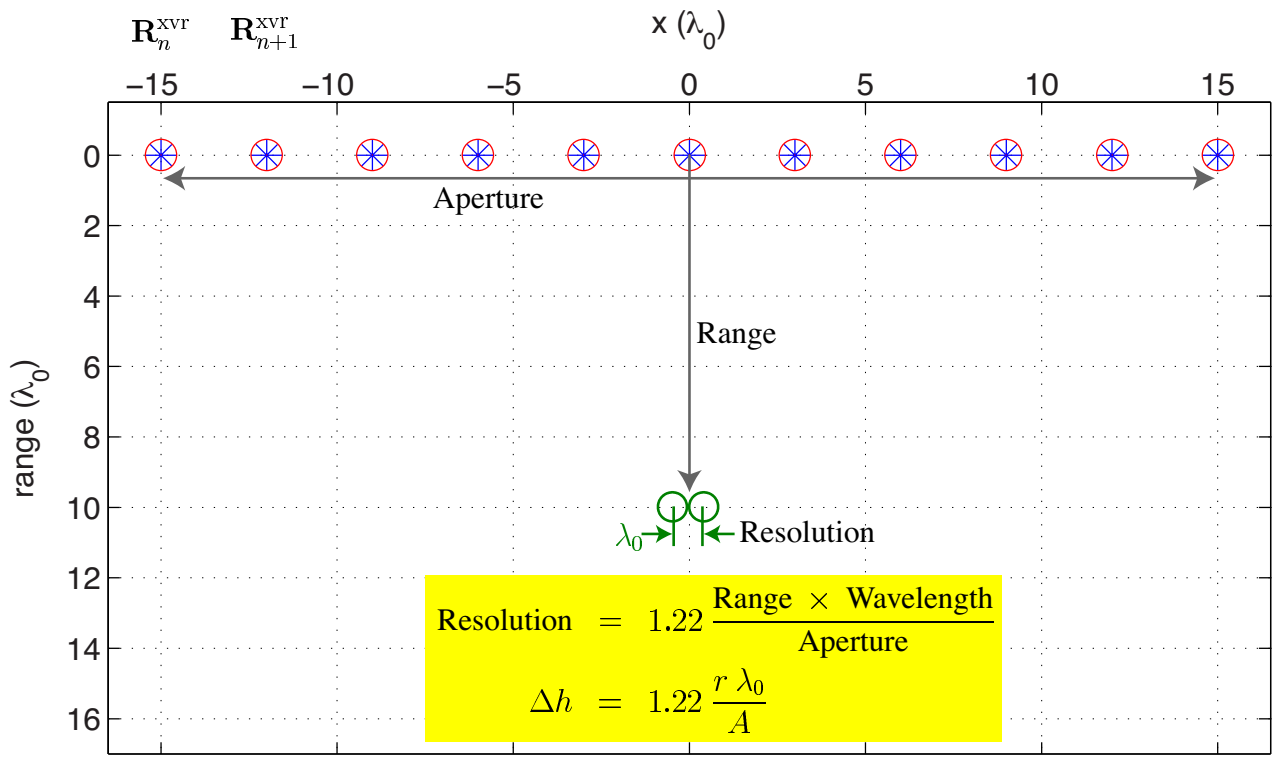

Figure 11: Geometry used in resolution descriptions.

The conclusion is aperture size contributes to resolution and increasing the number of elements reduces migration artifacts which obscure resolution.

Figure 14 compares multimonostatic with fully multistatic configurations. The additional perspective offered by the multistatic configuration achieves resolution with fewer elements while decreasing migration artifacts. 


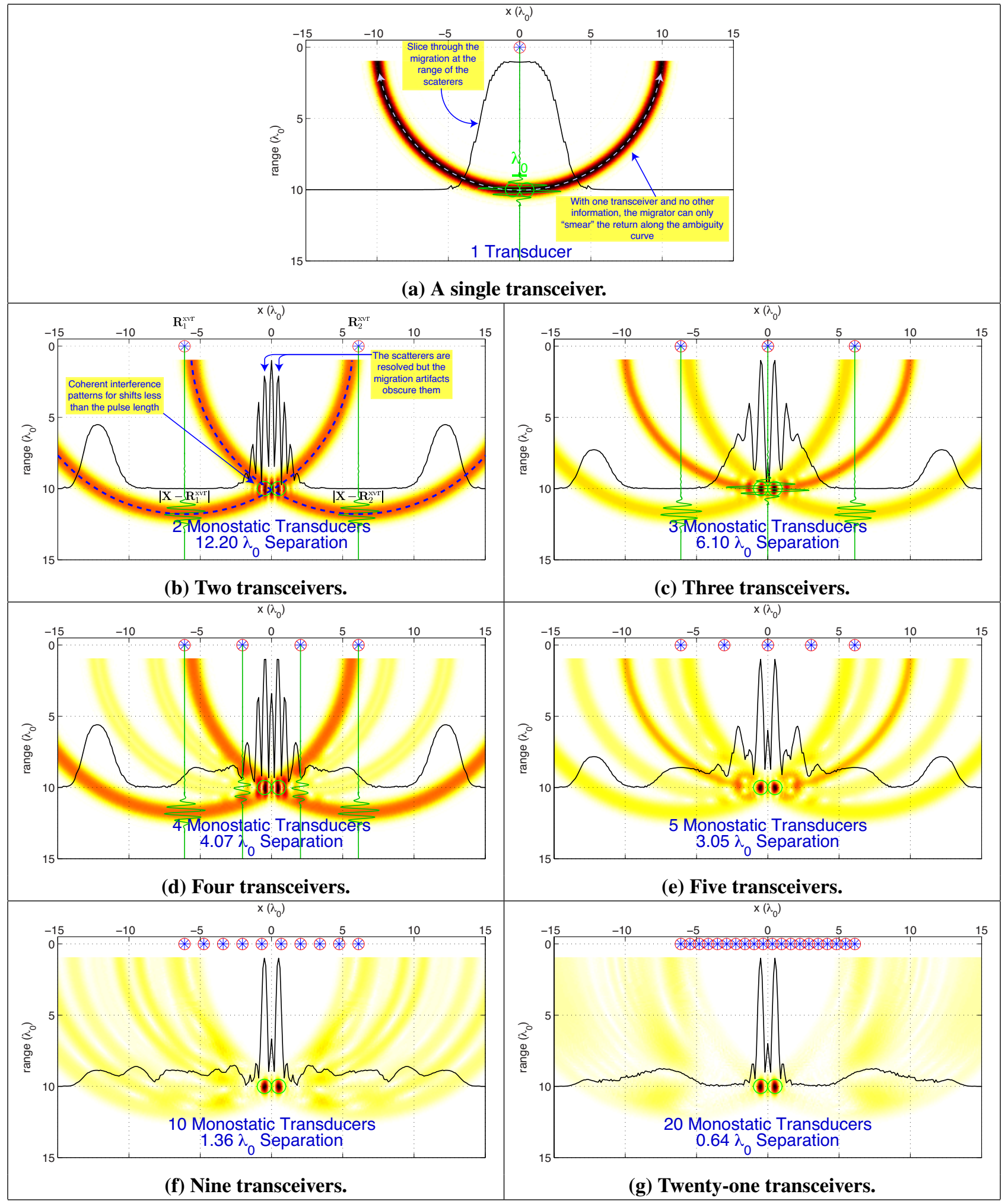

Figure 12: A graphical resolution study. Increasing the aperture, increases the resolution. Increasing the number of elements within the aperture, reduces the clutter; alternatively, it increases the signal-to-noise ratio. 


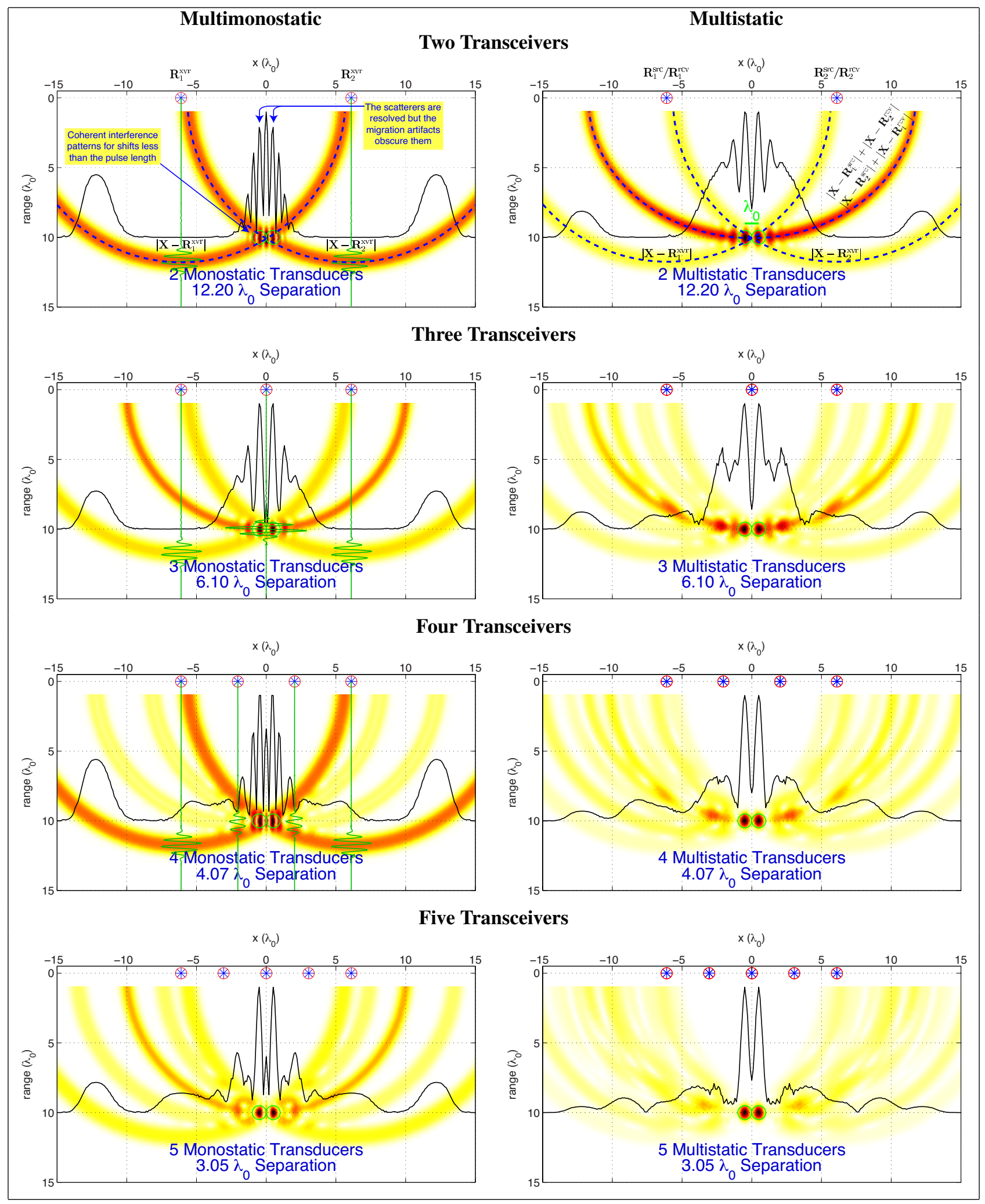

Figure 13: Comparison of multimonostatic with fully multistatic configurations. 


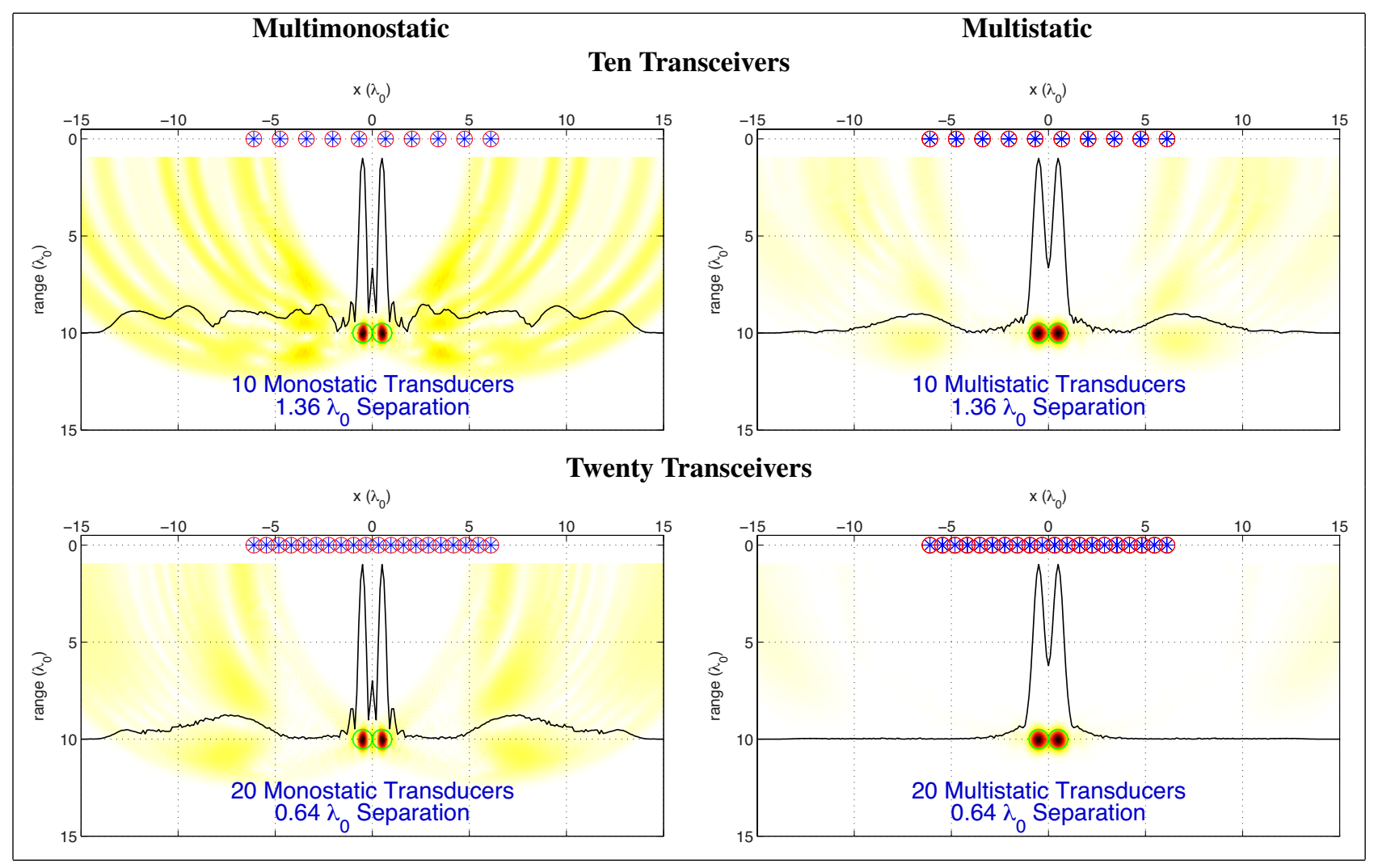

Figure 14: Comparison of multimonostatic with fully multistatic configurations. 


\section{Incident Field Propagation}

Solving the forward scattering problem requires solving for the background or incident field. The incident field is defined as that propagating environment in the absence of a scattering object. The forward propagation model is

$$
\left[\nabla^{2}-\frac{1}{c_{0}^{2}} \partial_{t}^{2}\right] \psi^{\mathrm{inc}}\left(\mathbf{r}, \mathbf{R}_{n}^{\mathrm{src}}, t\right)=-p\left(\mathbf{r}, \mathbf{R}_{n}^{\mathrm{src}}, t\right),
$$

where $p\left(\mathbf{r}, \mathbf{R}_{n}^{\mathrm{src}}, t\right)$ is the source distribution centered at $\mathbf{R}_{n}^{\mathrm{src}}$, and $\psi^{\mathrm{inc}}\left(\mathbf{r}, \mathbf{R}_{n}^{\mathrm{src}}, t\right)$ is the incident field in the volume, $V$. In anticipation of eventually considering a collection of $N_{\text {src }}$ sources located at $\left\{\mathbf{R}_{n}^{\text {src }}\right\}_{n=1}^{N_{\text {src }}}$, the source location is indexed by $n$.

The (background) Green function satisfies

$$
\left[\nabla^{2}-\frac{1}{c_{0}^{2}} \partial_{t}^{2}\right] G_{0}\left(\mathbf{r}, t ; \mathbf{r}^{\prime}, t^{\prime}\right)=-\delta\left(\mathbf{r}-\mathbf{r}^{\prime}, t-t^{\prime}\right)
$$

where the zero subscript is used to identify the homogeneous, free-space, background. The general solution to Eqn. (22) is [7]

$$
\begin{aligned}
\psi^{\mathrm{inc}}\left(\mathbf{r}, \mathbf{R}_{n}^{\mathrm{src}}, t\right)= & \int_{0}^{T} d t^{\prime} \int_{V} d \mathbf{r}^{\prime} G_{0}\left(\mathbf{r}, t ; \mathbf{r}^{\prime}, t^{\prime}\right) p\left(\mathbf{r}^{\prime}, \mathbf{R}_{n}^{\mathrm{src}}, t\right)+ \\
& \int_{0}^{T} d t^{\prime} \int_{S} d \Omega^{\prime}\left[\left.G_{0}\left(\mathbf{r}, t ; \mathbf{r}^{\prime}, t^{\prime}\right) \partial_{n^{\prime}} \psi^{\mathrm{inc}}\left(\mathbf{r}^{\prime}, \mathbf{R}_{n}^{\mathrm{src}}, t^{\prime}\right)\right|_{\mathbf{r}^{\prime}=S}-\right. \\
& \left.\left.\partial_{n^{\prime}} G_{0}\left(\mathbf{r}, t ; \mathbf{r}^{\prime}, t^{\prime}\right) \psi^{\mathrm{inc}}\left(\mathbf{r}^{\prime}, \mathbf{R}_{n}^{\mathrm{src}}, t^{\prime}\right)\right|_{\mathbf{r}^{\prime}=S}\right]+ \\
& \int_{V} d \mathbf{r}^{\prime} \frac{1}{c_{0}^{2}} G_{0}\left(\mathbf{r}, t ; \mathbf{r}^{\prime}, t^{\prime}\right) \psi^{\mathrm{inc}}\left(\mathbf{r}^{\prime}, \mathbf{R}_{n}^{\mathrm{src}}, 0\right)+ \\
& \partial_{t} \int_{V} d \mathbf{r}^{\prime} \frac{1}{c_{0}^{2}} G_{0}\left(\mathbf{r}, t ; \mathbf{r}^{\prime}, 0\right) \psi^{\mathrm{inc}}\left(\mathbf{r}^{\prime}, \mathbf{R}_{n}^{\mathrm{src}}, 0\right),
\end{aligned}
$$

where

$T$ : is the maximum time during which the field is to be evaluated. This chosen to be infinity.

$V:$ is the volume. This is chosen to be infinity.

$S:$ is the surface of the volume.

The following assumptions are made:

$$
\begin{aligned}
& \text { 1) } \quad \psi^{\text {inc }}\left(\mathbf{r}, \mathbf{R}_{n}^{\text {src }}, 0\right)=0 \quad \text { (no initial fields), } \\
& \text { 2) }\left.\psi^{\text {inc }}\left(\mathbf{r}, \mathbf{R}_{n}^{\text {src }}, t\right)\right|_{\mathbf{r}=S}=0 \quad \text { (no external fields on boundary), } \\
& \text { 3) }\left.\partial_{n} \psi^{\text {inc }}(\mathbf{r}, t)\right|_{\mathbf{r}=S} ^{\mathbf{r}=S}=0 \quad \text { (no external fields on boundary), } \\
& \text { 4) } \quad p\left(\mathbf{r}, \mathbf{R}_{n}^{\mathrm{src}}, t\right)=p(t) \delta\left(\mathbf{r}-\mathbf{R}_{n}^{\mathrm{src}}\right) \quad \text { (point sources located at } \mathbf{R}_{n}^{\mathrm{src}} \text { ). }
\end{aligned}
$$

These assumptions reduce Eqn. (24) to

$$
\psi^{\mathrm{inc}}\left(\mathbf{r}, \mathbf{R}_{n}^{\mathrm{src}}, t\right)=\int_{0}^{T} d t^{\prime} G_{0}\left(\mathbf{r}, t ; \mathbf{R}_{n}^{\mathrm{src}}, t^{\prime}\right) p\left(t^{\prime}\right),
$$

where the incident field expression has been expanded to indicate it is a result of the transmitter located at $\mathbf{R}_{n}^{\mathrm{src}}$. In the absence of scatterers, the background Green function is given by [7]

$$
G_{0}\left(\mathbf{r}, t ; \mathbf{r}^{\prime}, t^{\prime}\right)=\frac{\delta\left(t-t^{\prime}-\left|\mathbf{r}-\mathbf{r}^{\prime}\right| / c_{0}\right)}{4 \pi\left|\mathbf{r}-\mathbf{r}^{\prime}\right|}
$$


Which yields, when evaluated at the source position,

$$
G_{0}\left(\mathbf{r}, t ; \mathbf{R}_{n}^{\mathrm{src}}, t^{\prime}\right)=\frac{\delta\left(t-t^{\prime}-\left|\mathbf{r}-\mathbf{R}_{n}^{\mathrm{src}}\right| / c_{0}\right)}{4 \pi\left|\mathbf{r}-\mathbf{R}_{n}^{\mathrm{src}}\right|} .
$$

Combining Eqns. 26 and 28, results in

$$
\begin{aligned}
\psi^{\mathrm{inc}}\left(\mathbf{r}, \mathbf{R}_{n}^{\mathrm{src}}, t\right) & =\lim _{T \rightarrow \infty} \int_{0}^{T} d t^{\prime} \frac{\delta\left(t-t^{\prime}-\left|\mathbf{r}-\mathbf{R}_{n}^{\mathrm{src}}\right| / c_{0}\right)}{4 \pi\left|\mathbf{r}-\mathbf{R}_{n}^{\mathrm{src}}\right|} p\left(t^{\prime}\right), \\
& =\frac{1}{4 \pi\left|\mathbf{r}-\mathbf{R}_{n}^{\mathrm{src}}\right|} p\left(t-\frac{\left|\mathbf{r}-\mathbf{R}_{n}^{\mathrm{src}}\right|}{c_{0}}\right),
\end{aligned}
$$

which is a time-shifted (delayed) and scaled version of the original source. Observe the delay depends only upon the difference between the source and observer (eventually the receiver) location.

The incident field measured at the receiver located at $\mathbf{R}_{m}^{\mathrm{rcv}}$ is then given by

$$
\psi^{\mathrm{inc}}\left(\mathbf{R}_{m}^{\mathrm{rcv}}, \mathbf{R}_{n}^{\mathrm{src}}, t\right)=\frac{1}{4 \pi\left|\mathbf{R}_{m}^{\mathrm{rcv}}-\mathbf{R}_{n}^{\mathrm{src}}\right|} p\left(t-\frac{\left|\mathbf{R}_{m}^{\mathrm{rcv}}-\mathbf{R}_{n}^{\mathrm{src}}\right|}{c_{0}}\right) .
$$

\section{Scattered Field Propagation}

Forward propagation in the presence of scattering object(s) is analyzed in the temporal Fourier domain using the transforms as defined in Appendix A. Consider the wave equation

$$
\left[\nabla^{2}-\frac{n^{2}(\mathbf{r})}{c_{0}^{2}} \partial_{t}^{2}\right] \psi^{t o t}\left(\mathbf{r}, \mathbf{R}_{n}^{\mathrm{src}}, t\right)=-p\left(\mathbf{r}, \mathbf{R}_{n}^{\mathrm{src}}, t\right),
$$

in which an inhomogeneous background is modeled a spatially dependent refractive index,

$$
n(\mathbf{r}) \equiv \frac{c_{0}}{c(\mathbf{r})}
$$

Fourier transform Eqn. (31) to achieve

$$
\left[\nabla^{2}+k_{0}^{2} n^{2}(\mathbf{r})\right] \psi^{\mathrm{tot}}\left(\mathbf{r}, \mathbf{R}_{n}^{\mathrm{src}}, \omega\right)=-p\left(\mathbf{r}, \mathbf{R}_{n}^{\mathrm{src}}, \omega\right),
$$

where the background wavenumber is defined as

$$
k_{0} \equiv \frac{\omega}{c_{0}} .
$$

When solving the forward problem, it is frequently convenient to cast Eqn. (33) into an integral equation. Add $k_{0} \psi^{\mathrm{tot}}\left(\mathbf{r}, \mathbf{R}_{n}^{\mathrm{src}}, \omega\right)$ to both sides of Eqn. 33, and move the inhomogeneous term to the right hand side:

$$
\left[\nabla^{2}+k_{0}^{2}(\omega)\right] \psi^{\text {tot }}\left(\mathbf{r}, \mathbf{R}_{n}^{\mathrm{src}}, \omega\right)=-p(\mathbf{r})-\left[k_{0}^{2} n^{2}(\mathbf{r})-k_{0}^{2}\right] \psi^{\text {tot }}\left(\mathbf{r}, \mathbf{R}_{n}^{\mathrm{src}}, \omega\right) .
$$

Define the object function as

$$
o(\mathbf{r}) \equiv n^{2}(\mathbf{r})-1,
$$

and express Eqn. 34 as

$$
\left[\nabla^{2}+k_{0}^{2}(\omega)\right] \psi^{\mathrm{tot}}\left(\mathbf{r}, \mathbf{R}_{n}^{\mathrm{src}}, \omega\right)=-p\left(\mathbf{r}, \mathbf{R}_{n}^{\mathrm{src}}, \omega\right)-k_{0}^{2} o(\mathbf{r}) \psi^{\mathrm{tot}}\left(\mathbf{r}, \mathbf{R}_{n}^{\mathrm{src}}, \omega\right) .
$$


The first term on the right-hand side of Eqn. (36), $p\left(\mathbf{r}, \mathbf{R}_{n}^{\mathrm{src}}, \omega\right)$, is the primary source. The second, source-like, term on the right-hand side, $k_{0}^{2} o(\mathbf{r}) \psi^{\text {tot }}\left(\mathbf{r}, \mathbf{R}_{n}^{\text {src }}, \omega\right)$, is known as the secondary source. Green's theorem is used to cast the differential equation of Eqn. 36 into an integral equation [8],

$$
\begin{aligned}
\psi^{\text {tot }}\left(\mathbf{r}, \mathbf{R}_{n}^{\text {src }}, \omega\right)= & \underbrace{\int d \mathbf{r}^{\prime} G_{0}\left(\mathbf{r}, \omega ; \mathbf{r}^{\prime}, t^{\prime}\right) p\left(\mathbf{r}^{\prime}, \mathbf{R}_{n}^{\text {src }}, \omega\right)}_{\text {The incident field: } \psi^{\text {inc }}\left(\mathbf{r}, \mathbf{R}_{n}^{\text {src }}, \omega\right)}+ \\
& \underbrace{k_{0}^{2} \int d \mathbf{r}^{\prime} G_{0}\left(\mathbf{r}, \omega ; \mathbf{r}^{\prime}, t^{\prime}\right) o\left(\mathbf{r}^{\prime}\right) \psi^{\text {tot }}\left(\mathbf{r}^{\prime}, \mathbf{R}_{n}^{\text {src }}, \omega\right)}_{\text {The scattered field: } \psi^{\text {scat }}\left(\mathbf{r}, \mathbf{R}_{n}^{\text {src }}, \omega\right)} .
\end{aligned}
$$

where the background Green function satisfies

$$
\left[\nabla^{2}+k_{0}^{2}(\omega)\right] G_{0}\left(\mathbf{r}, \omega ; \mathbf{r}^{\prime}, 0\right)=-\delta\left(\mathbf{r}-\mathbf{r}^{\prime}\right)
$$

where

$$
G_{0}\left(\mathbf{r}, \omega ; \mathbf{r}^{\prime}, 0\right)=\frac{e^{i k_{0}\left|\mathbf{r}-\mathbf{r}^{\prime}\right|}}{4 \pi\left|\mathbf{r}-\mathbf{r}^{\prime}\right|}
$$

The primary, incident, or background field, defined as the solution to Eqn. (22), is

$$
\psi^{\mathrm{inc}}\left(\mathbf{r}, \mathbf{R}_{n}^{\mathrm{src}}, \omega\right) \equiv \int d \mathbf{r}^{\prime} G_{0}\left(\mathbf{r}, \omega ; \mathbf{r}^{\prime}, t^{\prime}\right) p\left(\mathbf{r}^{\prime}, \mathbf{R}_{n}^{\mathrm{src}}, \omega\right),
$$

so that Eqn. 37 reads

$$
\begin{aligned}
\psi^{\mathrm{tot}}\left(\mathbf{r}, \mathbf{R}_{n}^{\mathrm{src}}, \omega\right)= & \psi^{\mathrm{inc}}\left(\mathbf{r}, \mathbf{R}_{n}^{\mathrm{src}}, \omega\right)+ \\
& k_{0}^{2} \int d \mathbf{r}^{\prime} G_{0}\left(\mathbf{r}, \omega ; \mathbf{r}^{\prime}, t^{\prime}\right) o\left(\mathbf{r}^{\prime}\right) \psi^{\mathrm{tot}}\left(\mathbf{r}^{\prime}, \mathbf{R}_{n}^{\mathrm{src}}, \omega\right) .
\end{aligned}
$$

The scattered field is then defined as the difference between the total and incident fields,

$$
\begin{aligned}
\psi^{\mathrm{scat}}\left(\mathbf{r}, \mathbf{R}_{n}^{\mathrm{src}}, \omega\right) & \equiv \psi^{\mathrm{tot}}\left(\mathbf{r}, \mathbf{R}_{n}^{\mathrm{src}}, \omega\right)-\psi^{\mathrm{inc}}\left(\mathbf{r}, \mathbf{R}_{n}^{\mathrm{src}}, \omega\right) \\
& =k_{0}^{2} \int d \mathbf{r}^{\prime} G_{0}\left(\mathbf{r}, \omega ; \mathbf{r}^{\prime}, t^{\prime}\right) o\left(\mathbf{r}^{\prime}\right) \psi^{\mathrm{tot}}\left(\mathbf{r}^{\prime}, \mathbf{R}_{n}^{\mathrm{src}}, \omega\right) .
\end{aligned}
$$

Observe that with the primary field satisfying,

$$
\left[\nabla^{2}+k_{0}^{2}(\omega)\right] \psi^{\mathrm{inc}}\left(\mathbf{r}, \mathbf{R}_{n}^{\mathrm{src}}, \omega\right)=-p\left(\mathbf{r}, \mathbf{R}_{n}^{\mathrm{src}}, \omega\right),
$$

the scattered field obeys

$$
\left[\nabla^{2}+k_{0}^{2}\right] \psi^{\mathrm{scat}}\left(\mathbf{r}, \mathbf{R}_{n}^{\mathrm{src}}, \omega\right)=-k_{0}^{2} o(\mathbf{r}) \psi^{\mathrm{tot}}\left(\mathbf{r}, \mathbf{R}_{n}^{\mathrm{src}}, \omega\right),
$$

or alternatively,

$$
\left[\nabla^{2}+k_{0}^{2} n^{2}(\mathbf{r})\right] \psi^{\mathrm{scat}}\left(\mathbf{r}, \mathbf{R}_{n}^{\mathrm{src}}, \omega\right)=-k_{0}^{2} o(\mathbf{r}) \psi^{\mathrm{inc}}\left(\mathbf{r}, \mathbf{R}_{n}^{\mathrm{src}}, \omega\right),
$$

where Eqn. (35) has been used.

The integral equation of Eqn. (42) is non-linear in that the scattered field appears in both sides of the equation. The differential equation of Eqn. (45) offers the problem of having spatially dependent scattering operator. Exact analytic solutions for the scattered field exist only for a limited set of scattering objects. Numerical solutions are readily available but not appropriate for an arbitrary object tomographic inversion algorithm. Thus, we wish to simplify our forward model. In the following section the model is simplified under the Born approximation. 


\section{Born Approximation}

One potential method of simplifying Eqn. (45) prior to solving is to use a perturbation approach. Express the refractive index and scattered field as [9]

$$
\begin{aligned}
n^{2}(\mathbf{r}) & =\underbrace{n_{0}^{2}(\mathbf{r})}_{\equiv 1}+\underbrace{\epsilon n_{1}(\mathbf{r})+\epsilon^{2} n_{2}(\mathbf{r})+\cdots}_{\equiv \delta n(\mathbf{r})}, \\
\psi^{\text {scat }}\left(\mathbf{r}, \mathbf{R}_{n}^{\text {src }}, \omega\right) & =\psi_{0}^{\text {scat }}\left(\mathbf{r}, \mathbf{R}_{n}^{\text {src }}, \omega\right)+\epsilon \psi_{1}^{\text {scat }}\left(\mathbf{r}, \mathbf{R}_{n}^{\text {src }}, \omega\right)+\epsilon^{2} \psi_{2}^{\text {scat }}\left(\mathbf{r}, \mathbf{R}_{n}^{\text {src }}, \omega\right)+\cdots,
\end{aligned}
$$

respectively, substitute them into Eqn. (45), and equate like powers of $\epsilon$. Doing so yields the follow set of equations:

$$
\begin{aligned}
{\left[\nabla^{2}+k_{0}^{2}\right] \psi_{0}^{\text {scat }}\left(\mathbf{r}, \mathbf{R}_{n}^{\mathrm{src}}, \omega\right) } & =-k_{0}^{2} o(\mathbf{r}) \psi^{\mathrm{inc}}\left(\mathbf{r}, \mathbf{R}_{n}^{\mathrm{src}}, \omega\right), \\
{\left[\nabla^{2}+k_{0}^{2}\right] \psi_{1}^{\text {scat }}\left(\mathbf{r}, \mathbf{R}_{n}^{\mathrm{src}}, \omega\right) } & =-k_{0}^{2} n_{1}(\mathbf{r}) \psi_{0}^{\mathrm{scat}}\left(\mathbf{r}, \mathbf{R}_{n}^{\mathrm{src}}, \omega\right), \\
{\left[\nabla^{2}+k_{0}^{2}\right] \psi_{2}^{\text {scat }}\left(\mathbf{r}, \mathbf{R}_{n}^{\mathrm{src}}, \omega\right) } & =-k_{0}^{2}\left[n_{2}(\mathbf{r}) \psi_{0}^{\mathrm{scat}}\left(\mathbf{r}, \mathbf{R}_{n}^{\mathrm{src}}, \omega\right)-n_{1}(\mathbf{r}) \psi_{1}^{\mathrm{scat}}\left(\mathbf{r}, \mathbf{R}_{n}^{\mathrm{src}}, \omega\right)\right], \\
& \vdots \\
{\left[\nabla^{2}+k_{0}^{2}\right] \psi_{l}^{\text {scat }}\left(\mathbf{r}, \mathbf{R}_{n}^{\mathrm{src}}, \omega\right) } & =-k_{0}^{2} \sum_{m=0}^{l-1} n_{l-m}(\mathbf{r}) \psi_{m}^{\mathrm{scat}}\left(\mathbf{r}, \mathbf{R}_{n}^{\mathrm{src}}, \omega\right) .
\end{aligned}
$$

The scattering operator in the left hand side of Eqns. 47 through 50 is spatially independent. The Green function for the operator is that of Eqn. (38). It is used to cast Eqn. (47) into an integral equation to obtain the Born approximation to the scattered field,

$$
\psi_{B}^{\mathrm{scat}}\left(\mathbf{r}, \mathbf{R}_{n}^{\mathrm{src}}, \omega\right) \equiv \psi_{0}^{\mathrm{scat}}\left(\mathbf{r}, \mathbf{R}_{n}^{\mathrm{src}}, \omega\right)=k_{0}^{2} \int d \mathbf{r}^{\prime} G_{0}\left(\mathbf{r}, \omega ; \mathbf{r}^{\prime}, t^{\prime}\right) o\left(\mathbf{r}^{\prime}\right) \psi^{\mathrm{inc}}\left(\mathbf{r}^{\prime}, \mathbf{R}_{n}^{\mathrm{src}}, \omega\right) .
$$

Comparing this to Eqn. (42), it is seen the Born approximation effectively replaces the total field with the incident field, assuming a weak scattering approximation.

\subsection{Scattered Field Resulting from a Point Source}

The field resulting from a point source is given by Eqn. (29). Its temporal Fourier transform is

$$
\psi^{\text {inc }}\left(\mathbf{r}, \mathbf{R}_{n}^{\mathrm{src}}, \omega\right)=\frac{e^{i k_{0}\left|\mathbf{r}-\mathbf{R}_{n}^{\mathrm{src}}\right|}}{4 \pi \mid \mathbf{r}-\mathbf{R}_{n}^{\mathrm{src} \mid}} P(\omega) .
$$

The Fourier transform of the background Green function of Eqn. (27) is

$$
G_{0}\left(\mathbf{r}, \omega ; \mathbf{r}^{\prime}, t^{\prime}\right)=\frac{e^{i k_{0}\left|\mathbf{r}-\mathbf{r}^{\prime}\right|} e^{i \omega t^{\prime}}}{4 \pi\left|\mathbf{r}-\mathbf{r}^{\prime}\right|}
$$

Substituting Eqns. 52 and 53 into Eqn. (51), achieves

$$
\psi_{B}^{\mathrm{scat}}\left(\mathbf{r}, \mathbf{R}_{n}^{\mathrm{src}}, \omega\right)=P(\omega) \frac{k_{0}^{2} e^{i \omega t^{\prime}}}{(4 \pi)^{2}} \int d \mathbf{r}^{\prime} \frac{e^{i k_{0}\left(\left|\mathbf{r}-\mathbf{r}^{\prime}\right|+\left|\mathbf{r}^{\prime}-\mathbf{R}_{n}^{\mathrm{src}}\right|\right)}}{\left|\mathbf{r}-\mathbf{r}^{\prime}\right|\left|\mathbf{r}^{\prime}-\mathbf{R}_{n}^{\mathrm{src}}\right|} o\left(\mathbf{r}^{\prime}\right) .
$$

\subsection{Scattered Field from a Collection of Point Scatterers}

Consider a collection of point scatterers located at $\left\{\mathbf{X}_{j}\right\}_{j=1}^{J}$ with refractive indices $\left\{n_{j}\right\}_{j=1}^{J}$. The refractive index of Eqn. (31) is expressed as

$$
n^{2}(\mathbf{r})=1+\sum_{j=1}^{J}\left(n_{j}^{2}-1\right) \delta\left(\mathbf{r}-\mathbf{X}_{j}\right)
$$


Resulting in an object function (see Eqn. (35)) of

$$
o(\mathbf{r})=\sum_{j=1}^{J}\left(n_{j}^{2}-1\right) \delta\left(\mathbf{r}-\mathbf{X}_{j}\right) .
$$

Defining the point scattering amplitude as

$$
\tau_{j} \equiv n_{j}^{2}-1
$$

we arrive at the object distribution function,

$$
o(\mathbf{r})=\sum_{j=1}^{J} \tau_{j} \delta\left(\mathbf{r}-\mathbf{X}_{j}\right) .
$$

Substituting thee object distribution function into Eqn. (54) and integrating yields

$$
\psi_{B}^{\mathrm{scat}}\left(\mathbf{r}, \mathbf{R}_{n}^{\mathrm{src}}, \omega\right)=P(\omega) \frac{k_{0}^{2} e^{i k_{0} t^{\prime}}}{(4 \pi)^{2}} \sum_{j=1}^{J} \tau_{j} \frac{e^{i k_{0}\left(\left|\mathbf{r}-\mathbf{X}_{j}\right|+\left|\mathbf{X}_{j}-\mathbf{R}_{n}^{\mathrm{src}}\right|\right)}}{\left|\mathbf{r}-\mathbf{X}_{j}\right|\left|\mathbf{X}_{j}-\mathbf{R}_{n}^{\mathrm{src}}\right|} .
$$

Inverse Fourier transforming in time, we have

$$
\psi_{B}^{\mathrm{scat}}\left(\mathbf{r}, \mathbf{R}_{n}^{\mathrm{src}}, t\right)=\frac{1}{(4 \pi)^{2} c_{0}^{2}} \mathcal{F}^{-1}\{\underbrace{\omega^{2} P(\omega) e^{i \omega t^{\prime}}}_{I_{p}(\omega)} \sum_{j=1}^{J} \tau_{j} \underbrace{\frac{e^{i k_{0}\left(\left|\mathbf{r}-\mathbf{X}_{j}\right|+\left|\mathbf{X}_{j}-\mathbf{R}_{n}^{\mathrm{src}}\right|\right)}}{\left|\mathbf{r}-\mathbf{X}_{j}\right| \mid \mathbf{X}_{j}-\mathbf{R}_{n}^{\mathrm{src} \mid}}}_{I_{j}(\omega)}\} .
$$

Eqn. (60) is the product in Fourier space of two functions identified as $I_{p}(\omega)$ and $I_{j}(\omega)$. This represents a convolution in the time-domain. Consider the inverse Fourier transform of the first expression,

$$
\begin{aligned}
I_{1}\left(t-t^{\prime}\right) & =\mathcal{F}^{-1}\left\{I_{p}(\omega)\right\} \\
& =\frac{1}{2 \pi} \int_{-\infty}^{\infty} d \omega \omega^{2} P(\omega) e^{-i \omega\left(t-t^{\prime}\right)} \\
& =-\frac{1}{2 \pi} \frac{d^{2}}{d t^{2}} \int_{-\infty}^{\infty} d \omega P(\omega) e^{-i \omega\left(t-t^{\prime}\right)} \\
& =-\frac{1}{2 \pi} \frac{d^{2}}{d t^{2}} p\left(t-t^{\prime}\right) .
\end{aligned}
$$

The second expression is

$$
\begin{aligned}
I_{j}(t) & =\mathcal{F}^{-1}\left\{I_{j}(\omega)\right\} \\
& =\frac{1}{2 \pi\left|\mathbf{r}-\mathbf{X}_{j}\right|\left|\mathbf{X}_{j}-\mathbf{R}_{n}^{\mathrm{src}}\right|} \int_{-\infty}^{\infty} d \omega e^{i \omega\left(\left|\mathbf{r}-\mathbf{X}_{j}\right|+\left|\mathbf{X}_{j}-\mathbf{R}_{n}^{\mathrm{src}}\right|\right) / c_{0}} e^{-i \omega t}, \\
& =\frac{1}{\left|\mathbf{r}-\mathbf{X}_{j}\right| \mid \mathbf{X}_{j}-\mathbf{R}_{n}^{\mathrm{src} \mid}} \delta\left(t-\frac{\left|\mathbf{r}-\mathbf{X}_{j}\right|+\left|\mathbf{X}_{j}-\mathbf{R}_{n}^{\mathrm{src}}\right|}{c_{0}}\right) .
\end{aligned}
$$

The convolution is

$$
\begin{aligned}
\psi_{B}^{\mathrm{scat}}\left(\mathbf{r}, \mathbf{R}_{n}^{\mathrm{src}}, t\right) & =\frac{1}{8 \pi c_{0}^{2}} \sum_{j=1}^{J} \tau_{j} \int_{-\infty}^{\infty} d t^{\prime} I_{p}\left(t-t^{\prime}\right) I_{j}\left(t^{\prime}\right) \\
& =-\frac{1}{(4 \pi)^{2} c_{0}^{2}} \sum_{j=1}^{J} \frac{\tau_{j}}{\left|\mathbf{r}-\mathbf{X}_{j}\right|\left|\mathbf{X}_{j}-\mathbf{R}_{n}^{\mathrm{src}}\right|}\left[\frac{d^{2}}{d t^{2}} p\left(t-t^{\prime}\right)\right]_{t^{\prime}=\frac{\left|\mathbf{r}-\mathbf{x}_{j}\right|+\left|\mathbf{x}_{j}-\mathbf{R}_{n}^{\mathrm{src}}\right|}{c_{0}}} \\
& =-\frac{1}{(4 \pi)^{2} c_{0}^{2}} \sum_{j=1}^{J} \frac{\tau_{j}}{\left|\mathbf{r}-\mathbf{X}_{j}\right|\left|\mathbf{X}_{j}-\mathbf{R}_{n}^{\mathrm{src}}\right|} p^{\prime \prime}\left(t-\frac{\left|\mathbf{r}-\mathbf{X}_{j}\right|+\left|\mathbf{X}_{j}-\mathbf{R}_{n}^{\mathrm{src}}\right|}{c_{0}}\right)
\end{aligned}
$$


where the double prime on $p(\cdot)$ indicates the second derivative with respect to its argument.

\section{Full Scattering via Foldy-Lax}

Foldy-Lax scattering $[10,11,12]$ is a full wave scattering theory for point scatterers. It is exact within the constraint the scatterers are not "too close" (See Section 7.1). The derivation is as follows.

Return to the scattered field of Eqn. (42),

$$
\psi^{\mathrm{scat}}\left(\mathbf{r}, \mathbf{R}_{n}^{\mathrm{src}}, \omega\right)=k_{0}^{2} \int d \mathbf{r}^{\prime} G_{0}\left(\mathbf{r}, \omega ; \mathbf{r}^{\prime}, t^{\prime}\right) o\left(\mathbf{r}^{\prime}\right) \psi^{\mathrm{tot}}\left(\mathbf{r}^{\prime}, \mathbf{R}_{n}^{\mathrm{src}}, \omega\right),
$$

and substitute the object distribution function of Eqn. (58),

$$
o(\mathbf{r})=\sum_{j=1}^{J} \tau_{j} \delta\left(\mathbf{r}-\mathbf{X}_{j}\right)
$$

This yields

$$
\psi^{\mathrm{scat}}\left(\mathbf{r}, \mathbf{R}_{n}^{\mathrm{src}}, \omega\right)=k_{0}^{2} \sum_{j=1}^{J} \tau_{j} G_{0}\left(\mathbf{r}, \omega ; \mathbf{X}_{j}, t^{\prime}\right) \psi^{\mathrm{tot}}\left(\mathbf{X}_{j}, \mathbf{R}_{n}^{\mathrm{src}}, \omega\right) .
$$

To evaluate the scattered field of Eqn. (65), an expression is required for the total field evaluated at the scatters, $\psi^{\text {tot }}\left(\mathbf{X}_{j}, \mathbf{R}_{n}^{\text {src }}, \omega\right)$. Returning to Eqn. (41) and replacing the integral representation of the scattered field by Eqn. (65) achieves,

$$
\psi^{\text {tot }}\left(\mathbf{X}_{j}, \mathbf{R}_{n}^{\mathrm{src}}, \omega\right)=\psi^{\mathrm{inc}}\left(\mathbf{X}_{j}, \mathbf{R}_{n}^{\mathrm{src}}, \omega\right)+k_{0}^{2} \sum_{j^{\prime} \neq j}^{J} \tau_{j^{\prime}} G_{0}\left(\mathbf{X}_{j}, \omega ; \mathbf{X}_{j^{\prime}}, t^{\prime}\right) \psi^{\mathrm{tot}}\left(\mathbf{X}_{j^{\prime}}, \mathbf{R}_{n}^{\mathrm{src}}, \omega\right),
$$

where the self-scattering term has been removed because the Green function diverges when $\mathbf{X}_{j} \equiv \mathbf{X}_{j^{\prime}}$. This is ansatz motivated by the divergence. This represents a set of $J$ equations with $J$ unknown total fields, $\psi^{\text {tot }}\left(\mathbf{X}_{j^{\prime}}, \mathbf{R}_{n}^{\mathrm{src}}, \omega\right)$. The terms containing the unknowns are moved to the left hand side,

$$
\psi^{\mathrm{tot}}\left(\mathbf{X}_{j}, \mathbf{R}_{n}^{\mathrm{src}}, \omega\right)-k_{0}^{2} \sum_{j^{\prime} \neq j}^{J} \tau_{j^{\prime}} G_{0}\left(\mathbf{X}_{j}, \omega ; \mathbf{X}_{j^{\prime}}, t^{\prime}\right) \psi^{\mathrm{tot}}\left(\mathbf{X}_{j^{\prime}}, \mathbf{R}_{n}^{\mathrm{src}}, \omega\right)=\psi^{\mathrm{inc}}\left(\mathbf{X}_{j}, \mathbf{R}_{n}^{\mathrm{src}}, \omega\right),
$$

and express the result as a matrix equation,

$$
\left[\mathbf{I}-k_{0}^{2} \mathbf{T}\right] \psi_{n}^{\text {tot }}(\omega)=\psi_{n}^{\text {inc }}(\omega),
$$

with the following definitions,

$$
\begin{aligned}
& \mathbf{T} \equiv\left[\begin{array}{cccc}
0 & \tau_{2} G\left(\mathbf{X}_{1}, \omega ; \mathbf{X}_{2}, t^{\prime}\right) & \cdots & \tau_{J} G\left(\mathbf{X}_{1}, \omega ; \mathbf{X}_{J}, t^{\prime}\right) \\
\tau_{1} G\left(\mathbf{X}_{2}, \omega ; \mathbf{X}_{1}, t^{\prime}\right) & 0 & \cdots & \tau_{J} G\left(\mathbf{X}_{2}, \omega ; \mathbf{X}_{J}, t^{\prime}\right) \\
\vdots & \vdots & \vdots & \vdots \\
\tau_{1} G\left(\mathbf{X}_{J}, \omega ; \mathbf{X}_{1}, t^{\prime}\right) & \tau_{2} G\left(\mathbf{X}_{J}, \omega ; \mathbf{X}_{2}, t^{\prime}\right) & \cdots & 0
\end{array}\right], \\
& \psi_{n}^{\text {tot }}(\omega) \equiv\left[\begin{array}{c}
\psi^{\text {tot }}\left(\mathbf{X}_{1}, \mathbf{R}_{n}^{\mathrm{src}}, \omega\right) \\
\psi^{\text {tot }}\left(\mathbf{X}_{2}, \mathbf{R}_{n}^{\mathrm{src}}, \omega\right) \\
\vdots \\
\psi^{\text {tot }}\left(\mathbf{X}_{J}, \mathbf{R}_{n}^{\mathrm{src}}, \omega\right)
\end{array}\right] \\
& \psi_{n}^{\text {inc }}(\omega) \equiv\left[\begin{array}{c}
\psi^{\text {inc }}\left(\mathbf{X}_{1}, \mathbf{R}_{n}^{\mathrm{src}}, \omega\right) \\
\psi^{\text {inc }}\left(\mathbf{X}_{2}, \mathbf{R}_{n}^{\text {scc }}, \omega\right) \\
\vdots \\
\psi^{\text {inc }}\left(\mathbf{X}_{J}, \mathbf{R}_{n}^{\mathrm{src}}, \omega\right)
\end{array}\right]
\end{aligned}
$$


The solution is

$$
\begin{aligned}
\psi_{n}^{\text {tot }}(\omega) & =\left[I-k_{0}^{2} \mathbf{T}\right]^{-1} \psi_{n}^{\text {inc }}(\omega) \\
& =\left[I+k_{0}^{2} \mathbf{T}+k_{0}^{4}(\mathbf{T})^{2}+k_{0}^{6}(\mathbf{T})^{3}+\cdots\right] \psi_{n}^{\text {inc }}(\omega)
\end{aligned}
$$

which is back-substituted into Eqn. (65) to obtain the scattered field spectrum.

For the total field of Eqn. (72) to be physical, the series expansion of $I-k_{0}^{2} \mathbf{T}$ in Eqn. (73) must converge. Thus, we require [13]:

$$
k_{0}^{2}\|\mathbf{T}\|_{2}<1
$$

for stability.

\subsection{Stability Analysis of Foldy-Lax}

The stability of the Foldy-Lax model depends upon the convergence of the series in Eqn. (73). Convergence is ensured by constraining the magnitude of the scatterers. Eqn. (74) is imposed as a constraint:

$$
k_{0}^{2}\|\mathbf{T}\|_{2}<1
$$

It is shown in [13],

$$
\|\mathbf{T}\|_{2} \leq N_{\text {src }} \max _{j j^{\prime}}\left|\tau_{j^{\prime}} G_{0}\left(\mathbf{X}_{j}, \omega ; \mathbf{X}_{j^{\prime}}, t^{\prime}\right)\right|
$$

Extracting the maximum scattering strength, $\tau_{\max } \equiv \max _{j^{\prime}}\left|\tau_{j^{\prime}}\right|$, and observing the magnitude of the free space Green function of Eqn. (53) obeys:

$$
\left|G_{0}\left(\mathbf{X}_{j}, \omega ; \mathbf{X}_{j^{\prime}}, t^{\prime}\right)\right| \leq \frac{1}{4 \pi\left|\mathbf{X}_{j}-\mathbf{X}_{j^{\prime}}\right|} \leq \frac{1}{4 \pi R_{\min }}
$$

where

$$
R_{\min } \equiv \min _{j j^{\prime}, j \neq j^{\prime}}\left|\mathbf{X}_{j}-\mathbf{X}_{j^{\prime}}\right|
$$

Eqn. (75) is expressed as

$$
\|\mathbf{T}\|_{2}<\frac{N_{\text {src }} \max _{j^{\prime}}\left|\tau_{j^{\prime}}\right|}{4 \pi k_{0}^{2} R_{\min }}
$$

which is required to be less than one,

$$
\frac{N_{\mathrm{src}} \max _{j^{\prime}}\left|\tau_{j^{\prime}}\right|}{4 \pi k_{0}^{2} R_{\min }}<1
$$

Thus, the constraint on the maximum scattering strength is

$$
\max _{j^{\prime}}\left|\tau_{j^{\prime}}\right|<\frac{4 \pi k_{0}^{2} R_{\min }}{N_{\mathrm{src}}}
$$

that is, the scatterers may not be "too close" together. 


\section{Delay, Scale, \& Sum Beamforming: Migration}

Five expressions involving the scattered field have been derived:

1. An integral expression for the scattered field from Eqn. (42),

$$
\psi^{\mathrm{scat}}\left(\mathbf{r}, \mathbf{R}_{n}^{\mathrm{src}}, \omega\right)=k_{0}^{2} \int d \mathbf{r}^{\prime} G_{0}\left(\mathbf{r}, \omega ; \mathbf{r}^{\prime}, t^{\prime}\right) o\left(\mathbf{r}^{\prime}\right) \psi^{\mathrm{tot}}\left(\mathbf{r}^{\prime}, \mathbf{R}_{n}^{\mathrm{src}}, \omega\right),
$$

which can be difficult to solve as the total field contains the scattered field.

2. The differential equation equivalent to either Eqn. (42) or Eqn. (82) in Eqn. (44),

$$
\left[\nabla^{2}+k_{0}^{2}\right] \psi^{\mathrm{scat}}\left(\mathbf{r}, \mathbf{R}_{n}^{\mathrm{src}}, \omega\right)=-k_{0}^{2} o(\mathbf{r}) \psi^{\mathrm{tot}}\left(\mathbf{r}, \mathbf{R}_{n}^{\mathrm{src}}, \omega\right) .
$$

3. The Born approximation of Eqn. (51),

$$
\psi_{B}^{\mathrm{scat}}\left(\mathbf{r}, \mathbf{R}_{n}^{\mathrm{src}}, \omega\right)=k_{0}^{2} \int d \mathbf{r}^{\prime} G_{0}\left(\mathbf{r}, \omega ; \mathbf{r}^{\prime}, t^{\prime}\right) o\left(\mathbf{r}^{\prime}\right) \psi^{\mathrm{inc}}\left(\mathbf{r}^{\prime}, \mathbf{R}_{n}^{\mathrm{src}}, \omega\right) .
$$

4. The Born approximation for point scatterers of Eqn. (63),

$$
\psi_{B}^{\text {scat }}\left(\mathbf{r}, \mathbf{R}_{n}^{\mathrm{src}}, t\right)=-\frac{1}{\left(4 \pi c_{0}\right)^{2}} \sum_{j=1}^{J} \frac{\tau_{j}}{\left|\mathbf{r}-\mathbf{X}_{j}\right|\left|\mathbf{X}_{j}-\mathbf{R}_{n}^{\mathrm{src}}\right|} p^{\prime \prime}\left(t-\frac{\left|\mathbf{r}-\mathbf{X}_{j}\right|+\left|\mathbf{X}_{j}-\mathbf{R}_{n}^{\mathrm{src}}\right|}{c_{0}}\right) .
$$

5. And the Foldy-Lax full-wave point scatterer scattering solution of Eqns. 65 and 72,

$$
\begin{aligned}
\psi^{\mathrm{scat}}\left(\mathbf{r}, \mathbf{R}_{n}^{\mathrm{src}}, \omega\right) & =k_{0}^{2} \sum_{j=1}^{J} \tau_{j} G_{0}\left(\mathbf{r}, \omega ; \mathbf{X}_{j}, t^{\prime}\right) \psi^{\mathrm{tot}}\left(\mathbf{X}_{j}, \mathbf{R}_{n}^{\mathrm{src}}, \omega\right) \\
\psi_{n}^{\mathrm{tot}}(\omega) & =\left[I-k_{0}^{2} \mathbf{T}\right]^{-1} \psi_{n}^{\mathrm{inc}}(\omega) .
\end{aligned}
$$

To demonstrate the concept of delay and sum beamforming, consider the scattered field approximation of Item (4) in Eqn. (85), when measured at a collection of receivers located at $\left\{\mathbf{R}_{m}^{\mathrm{rcv}}\right\}_{m=1}^{N_{\mathrm{rcv}}}$ :

$$
\psi_{B}^{\mathrm{scat}}\left(\mathbf{R}_{m}^{\mathrm{rcv}}, \mathbf{R}_{n}^{\mathrm{src}}, t\right)=-\frac{1}{\left(4 \pi c_{0}\right)^{2}} \sum_{j=1}^{J} \frac{\tau_{j}}{\alpha_{m j n}} p^{\prime \prime}\left(t-t_{m j n}\right),
$$

where the delay and range scaling have been expressed most generally as $t_{m n}$ and $\alpha_{m n}$, respectively. Note, this represents a fully multistatic system in that the transducers are explicitly spatially diverse. In a homogeneous medium the time delay and range scale are

$$
t_{m j n} \equiv \frac{\left|\mathbf{R}_{m}^{\mathrm{rcv}}-\mathbf{X}_{j}\right|+\left|\mathbf{X}_{j}-\mathbf{R}_{n}^{\mathrm{src}}\right|}{c_{0}}
$$

and the scale

$$
\alpha_{m j n} \equiv\left|\mathbf{R}_{m}^{\mathrm{rcv}}-\mathbf{X}_{j}\right|\left|\mathbf{X}_{j}-\mathbf{R}_{n}^{\mathrm{src}}\right|,
$$

respectively. When migrating through inhomogeneous media, the expressions for delay and scale may be more complicated and, in fact, may lack a closed form.

Eqn. (88) represents a set of measurements identified as the multistatic data matrix (MSDM). Rigorously, the MSDM is derived from the measured field via

$$
v_{m n}^{\mathrm{scat}}(t)=T_{n}^{\mathrm{rcv}}(\mathbf{r}, t) * \psi^{\mathrm{scat}}\left(\mathbf{R}_{m}^{\mathrm{rcv}}, \mathbf{R}_{n}^{\mathrm{src}}, t\right)+n(t),
$$


as shown in Figure 1, however for the present, the receiver transfer function is taken to be $T_{n}^{\mathrm{xvr}}(\mathbf{r}, t) \equiv 1$ and any noise ignored, $n(t) \equiv 0$. With these assumptions, the MSDM is identical to the scattered field observed at the receiver:

$$
v_{m n}^{\mathrm{scat}}(t) \equiv \psi^{\mathrm{scat}}\left(\mathbf{R}_{m}^{\mathrm{rcv}}, \mathbf{R}_{n}^{\mathrm{src}}, t\right) .
$$

Note, in multimonostatic systems, $m \equiv n$, and the MSDM is a multimonostatic data matrix (MMDM) expressed as $v_{n}^{\text {scat }}(t)$.

Migration assumes or "guesses at" a scatterer location know as a focal point, $\mathbf{r}_{\mathrm{f}}=\left(x_{\mathrm{f}}, y_{\mathrm{f}}, z_{\mathrm{f}}\right)$, and reverses the delay and scaling of the forward scattering and propagation. The delay and range scale to the focal point are

$$
t_{m f n} \equiv \frac{\left|\mathbf{R}_{m}^{\mathrm{rcv}}-\mathbf{r}_{\mathrm{f}}\right|+\left|\mathbf{r}_{\mathrm{f}}-\mathbf{R}_{n}^{\mathrm{src}}\right|}{c_{0}}
$$

and

$$
\alpha_{m f n} \equiv\left|\mathbf{R}_{m}^{\mathrm{rcv}}-\mathbf{r}_{\mathrm{f}}\right|\left|\mathbf{r}_{\mathrm{f}}-\mathbf{R}_{n}^{\mathrm{src}}\right|,
$$

respectively. The shifted and scaled returns are summed with the assumption returns from a scatterer will sum coherently whereas returns from non-scattering center volumes will sum destructively. Finally, the sum is evaluated at the time of the pulse peak, $t_{\text {peak }}$, as identified in Figure 2. Mathematically, this is expressed as

$$
\hat{o}_{\mathrm{mig}}\left(\mathbf{r}_{\mathrm{f}}\right)=\frac{1}{N_{\mathrm{src}} N_{\mathrm{rcv}}} \sum_{n=1}^{N_{\mathrm{src}}} \sum_{m=1}^{N_{\mathrm{rcv}}} \alpha_{m \mathrm{fn}} v_{m n}^{\text {scat }}\left(t_{\mathrm{peak}}+t_{m \mathrm{f} n}\right),
$$

where $\hat{o}_{\text {mig }}\left(\mathbf{r}_{\mathrm{f}}\right)$ is an estimate of the scattering object achieved through migration. Substituting Eqn. (88), results in

$$
\hat{o}_{\mathrm{mig}}\left(\mathbf{r}_{\mathrm{f}}\right)=-\frac{1}{\left(4 \pi c_{0}\right)^{2} N_{\mathrm{src}} N_{\mathrm{rcv}}} \sum_{n=1}^{N_{\mathrm{src}}} \sum_{m=1}^{N_{\mathrm{rcv}}} \sum_{j=1}^{J} \tau_{j} \frac{\alpha_{m \mathrm{f} n}}{\alpha_{m j n}} p^{\prime \prime}\left(t_{\mathrm{peak}}-t_{m j n}+t_{m f n}\right) .
$$

Three cases must be considered,

1. When the time shift, $\left(t_{m j n}-t_{m f n}\right)$, exceeds the pulse length, the contribution due to the pulse derivative is zero, $p^{\prime \prime}\left(t_{\text {peak }}-t_{m j n}+t_{m f n}\right) \equiv 0$;

2. When the focal point coincides with the $j$-th scatterer, $\mathbf{r}_{\mathrm{f}} \equiv \mathbf{X}_{j}$, Eqn. (96) reduces to

$$
\begin{aligned}
& \hat{o}_{\mathrm{mig}}\left(\mathbf{X}_{j}\right)=-\frac{1}{\left(4 \pi c_{0}\right)^{2} N_{\mathrm{src}} N_{\mathrm{rcv}}} \times \\
& \sum_{n=1}^{N_{\text {src }}} \sum_{m=1}^{N_{\text {rcv }}}\left[\tau_{j} p^{\prime \prime}\left(t_{\text {peak }}\right)+\sum_{j^{\prime} \neq j}^{J} \tau_{j^{\prime}} \frac{\alpha_{m f n}}{\alpha_{m j^{\prime} n}} p^{\prime \prime}\left(t_{\text {peak }}-t_{m j^{\prime} n}+t_{m f n}\right)\right], \\
& =-\frac{1}{\left(4 \pi c_{0}\right)^{2}} \times \\
& {\left[\tau_{j} p^{\prime \prime}\left(t_{\mathrm{peak}}\right)+\frac{1}{N_{\mathrm{src}} N_{\mathrm{rcv}}} \sum_{n=1}^{N_{\mathrm{src}}} \sum_{m=1}^{N_{\mathrm{rcv}}} \sum_{j^{\prime} \neq j}^{J} \tau_{j^{\prime}} \frac{\alpha_{m \mathrm{f} n}}{\alpha_{m j^{\prime} n}} p^{\prime \prime}\left(t_{\mathrm{peak}}-t_{m j^{\prime} n}+t_{m \mathrm{f} n}\right)\right] .}
\end{aligned}
$$

For scatterers with a separation greater than a pulse length, the triple sum is incoherent and negligible.

3. For shifts less than a pulse length, the migration yields coherent interference patterns. 


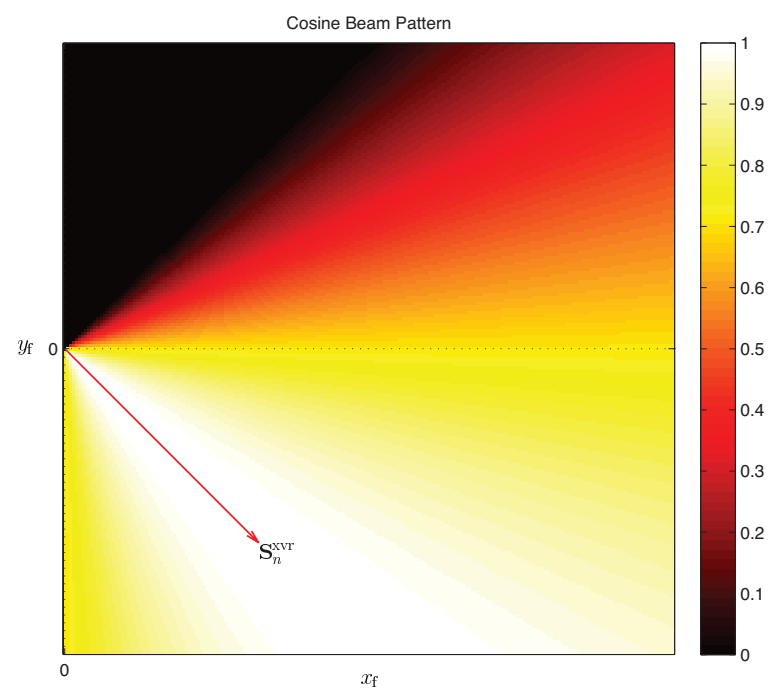

Figure 15: An example of a cosine beam pattern, $\frac{\mathbf{r}_{\mathrm{f}} \cdot \mathbf{S}_{n}^{\mathrm{rxvr}}}{\left|\mathbf{r}_{\mathrm{f}}\right|}$ as a function of a collection of planar focal points for an antenna heading of $\mathbf{S}_{n}^{\mathrm{rxvr}}$.

\subsection{Imposition of a Beam Pattern}

The derivation of the previous section assumes the sources and receivers are isotropic: they have a uniform beam pattern. This is not practically accurate. As a correction, an empirically based cosine beam pattern is imposed on the measured time series. Let $\left\{\mathbf{S}_{n}^{\mathrm{src}}\right\}_{n=1}^{N_{\mathrm{src}}}$ and $\left\{\mathbf{S}_{m}^{\mathrm{rcv}}\right\}_{m=1}^{N_{\mathrm{rcv}}}$ be the source and receiver heading unit vectors, respectively. The cosine beam pattern at each focal point is

$$
b_{m f n}=\left(\frac{\mathbf{r}_{\mathrm{f}} \cdot \mathbf{S}_{m}^{\mathrm{rcv}}}{\left|\mathbf{r}_{\mathrm{f}}\right|}\right)\left(\frac{\mathbf{r}_{\mathrm{f}} \cdot \mathbf{S}_{n}^{\mathrm{src}}}{\left|\mathbf{r}_{\mathrm{f}}\right|}\right)
$$

which is imposed on Eqn. (95) as

$$
\hat{o}_{\mathrm{mig}}\left(\mathbf{r}_{\mathrm{f}}\right)=\frac{1}{N_{\mathrm{src}} N_{\mathrm{rcv}}} \sum_{n=1}^{N_{\mathrm{src}}} \sum_{m=1}^{N_{\mathrm{rcv}}} \alpha_{m \mathrm{f} n} b_{m \mathrm{f} n} v_{m n}^{\mathrm{scat}}\left(t_{\text {peak }}+t_{m \mathrm{f} n}\right) .
$$

\subsection{Migration in a Planar Layer Medium}

The migration equation of Eqn. (95) requires the time delay, $t_{m f n}$, and range scale, $\alpha_{m f n}$, from the source to the focal point, and the focal point to the receiver. The method of computing or estimating the delay and scale are independent of the migration. Regardless of the background Green function, if they can be achieved, a migration can be performed.

Migration in a two medium space, planar layer or otherwise, is challenged by the issue of refraction at the interface. Ray traces must be performed from the transceivers to the focal points in order to estimate accurate delays and range scales. Figure 16 presents the case of a ray trace in a volume enclosing a two planar layer medium with refractive indices $n_{1}$ and $n_{2}$. A ray is "shot" from the source located at $\mathbf{R}_{n}^{\text {src }}$. It intercepts the surface at the point $\mathbf{p}$ and refracts in a manner to intercept the focal point $\mathbf{r}_{\mathrm{f}}$. The process is repeated for the "scattered" ray from the focal point to the receiver located at $\mathbf{R}_{m}^{\mathrm{rcv}}$. Snell's Law $[6,8,14]$ governs refraction at interfaces:

$$
n_{1} \sin \theta_{1}=n_{2} \sin \theta_{2},
$$

where $n_{1}$ and $n_{2}$ are the refractive indices of the top and bottom layers, respectively, and $\theta_{1}$ and $\theta_{2}$ are the incident and transmitted ray normal angles as shown in Figure 17. The details of solving Eqn. (100) at a focal point are presented in Appendix C. 


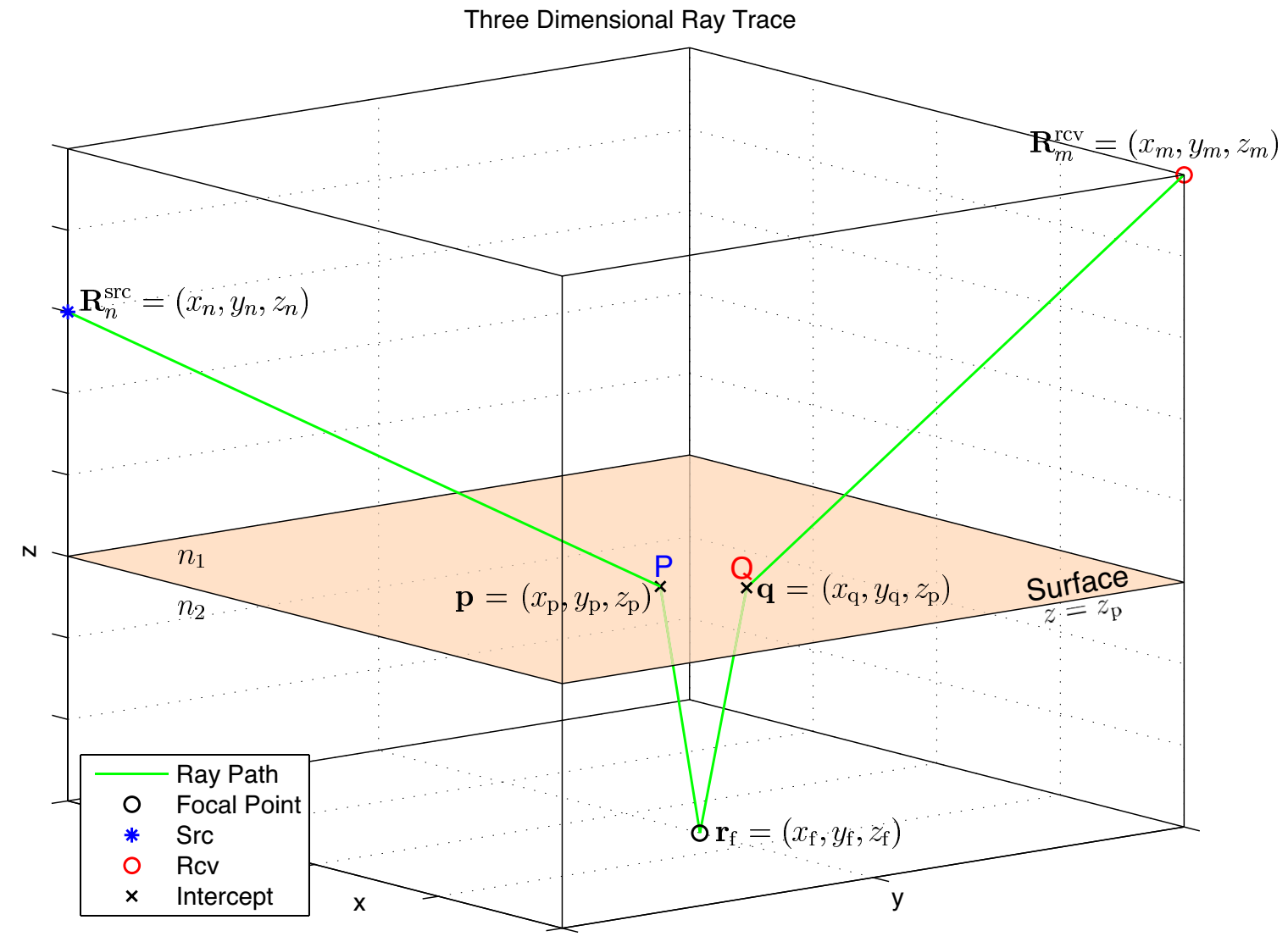

Figure 16: Two planar ray trace scenario. A ray is "shot" from the source located at $\mathbf{R}_{n}^{\mathrm{src}}$. It intercepts the surface at the point $\mathbf{p}$ and refracts in a manner to intercept the focal point $\mathbf{r}_{\mathrm{f}}$. The process is repeated for the "scattered" ray from the focal point to the receiver located at $\mathbf{R}_{m}^{\mathrm{rev}}$ through the surface intercept point $\mathbf{q}$. 


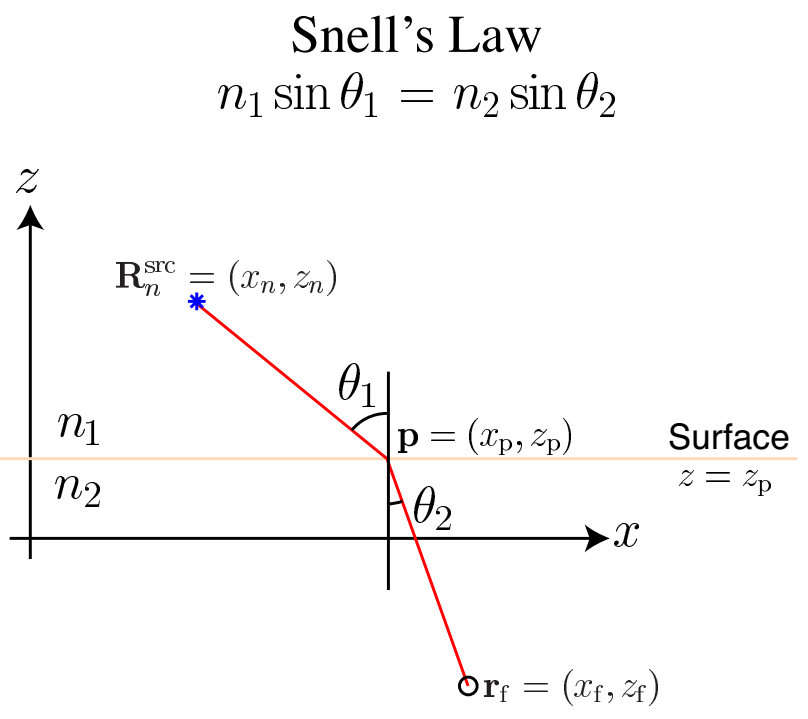

Figure 17: Snell's law. 


\section{Examples}

Four examples are presented which demonstrate delay, scale, and sum migration of measured wave data:

- Section 9.1 presents data collected on a wire-under-tension phantom in which the wires were arranged in a logarithmic spiral. Twenty-nine wires were $\frac{14}{1000}$ inch $(0.036 \mathrm{~mm})$ steel, one was $\frac{7}{1000}$ inch $(0.018 \mathrm{~mm})$ nylon, and one was missing (a steel wire snapped while being pulled taut). These diameters are at least an order of magnitude less than the pulse spectral peak wavelength. The experiment provides an example of remotely identifying a target (the nylon wire) in clutter (the steel wires). The measurement system was an annular array in which the transmitting and receiving transducers rotated independently along a $15 \mathrm{~cm}$ diameter annulus. The transmitter successively occupied 360 discrete locations with a one degree increment. Due to the mechanical obstruction of the transmitter, the receiver measured scattered field data at 320 locations with a one degree increment. This models imaging in clutter as well as the "As Built" versus "As Designed" problem with a multibistatic with variable offset system.

- Section 9.2 presents data collected on an aluminum block glued onto a copper block in which a hole was drilled. This provides an example of locating a defect in a two planar layer medium. Migrating in this environment requires accounting for the field refraction at the interface. A migration is performed using Snell's Law at the interface. This example serves as a model of imaging objects buried in a planar layered medium with a multistatic system.

- Adopting the philosophy that "a wave behaves as a wave", independent of the material physics, Section 9.3 presents an aluminum pole resolution phantom buried in dry, loamy sand. A wide band, ground penetrating, radar is used in a multimonostatic operating mode to image the subsurface environment. The mathematics of the forward scattering and migration remain the same as in the acoustic case only the physical properties of the refractive index change.

- Section 9.4 presents data on an aluminum block with two drilled holes representing "buried" objects. The linear ultrasonic transmitting and receiving arrays are placed, orthogonally, on adjacent sides of the block. This multistatic system models offset vertical seismic profiling.

\subsection{Wire Phantom}

A wire phantom consisting of thirty $\frac{14}{1000}$ inch $(0.036 \mathrm{~mm})$ steel wires and one $\frac{7}{1000}$ inch $(0.018 \mathrm{~mm})$ nylon wire pulled taut between two guide plates was designed and built to serve as a two-and-half dimensional scattering object. The wires were threaded through holes drilled into the guide plates which were pulled apart, placing the wires under tension. The distribution of the holes defined the cross-sectional wire pattern which followed a logarithmic spiral described by

$$
\mathbf{r}\left(\theta_{n}\right)=a\left[\cos \left(\theta_{n}\right), \sin \left(\theta_{n}\right)\right] e^{b \theta_{n}}
$$

where

$$
\begin{aligned}
v_{0} & \equiv 1500 \mathrm{~meters} / \mathrm{second} & & \text { is the assumed background water velocity, } \\
f_{0} & \equiv 1.6 \mathrm{MHz} & & \text { is the approximate peak insonifying frequency, } \\
\lambda_{0} & \equiv v_{0} / f_{0}=0.94 \mathrm{~mm} & & \text { is the insonifying wavelength, } \\
a & \equiv \lambda_{0} & & \text { is the initial radius and, } \\
b & \equiv 10 \pi / 180 & & \text { is the spiral growth rate, } \\
\theta_{n} & \equiv\{n \Delta \theta\}_{n=0}^{N-1} & & \text { are the angular locations of the wires, } \\
\Delta \theta & \equiv 30 \pi / 180 & & \text { is the angular increment, } \\
N & \equiv 31 & & \text { is the number of wires. }
\end{aligned}
$$

The incident pulse consisted of two cycles of a sinusoid with a center frequency of $1.6 \mathrm{MHz}$. The wavelength was approximately $0.94 \mathrm{~mm}$. Thus the wires may be considered "thin" and near perfect point scatterers. A photograph of a guile plate is shown in Figure 18 and two views of the assembled phantom in Figure 19. The phantom was placed in an 

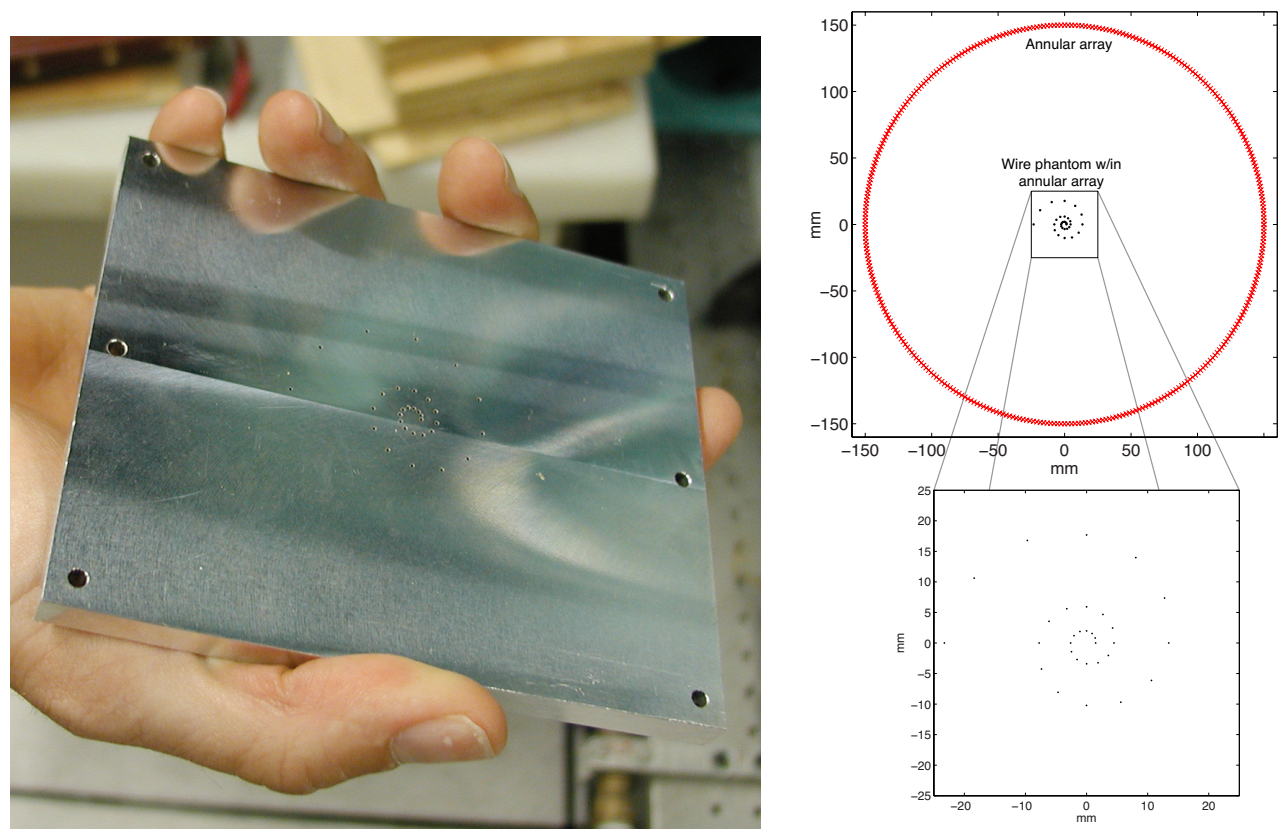

Figure 18: Photograph of the wire phantom guide plate and a schematic of the wire phantom within the annular array.

annular acoustic array submerged in a water bath. The radially inward-looking array had a radius of $150 \mathrm{~mm}$. A source and receiving transducer, rotating in a common plane, were used to collect and synthesize a fully multistatic data set although the system, itself, was multibistatic with variable offset. The source rotated about the center at one degree increments launching the incident field into the (water) medium. The receiver would rotate at one degree increments measuring the total field. The mechanical movement of the receiver was limited to \pm 20 degrees about each source transducer position: the transducers' support arms limited the rotational excursion of the receiver. Thus, there is a 40 degree received total field "gap" at each source location. Thus the multibistatic nature of the system: not all receiver locations measured fields from all source locations. 


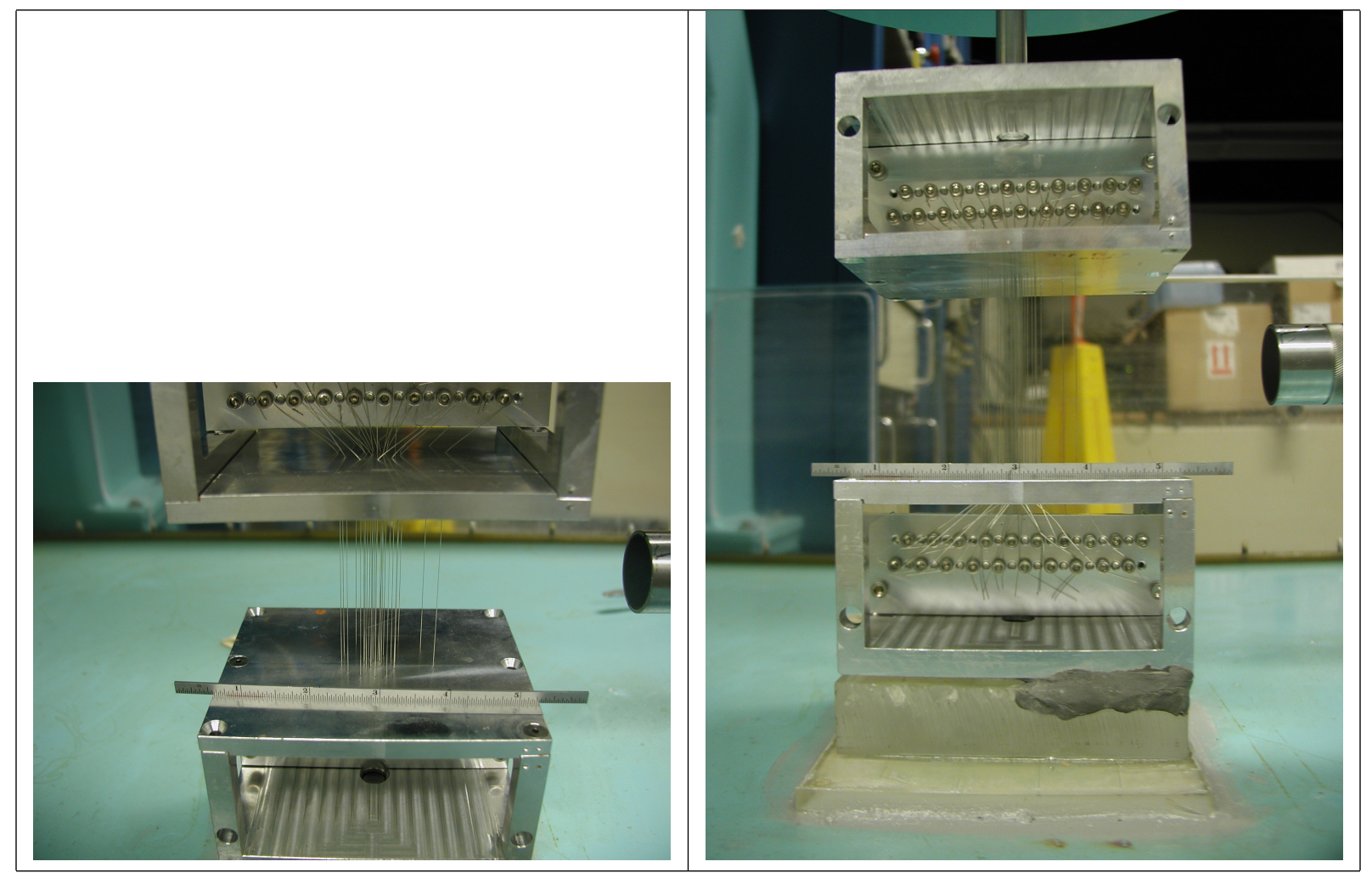

Figure 19: Two views of the assembled wire phantom.

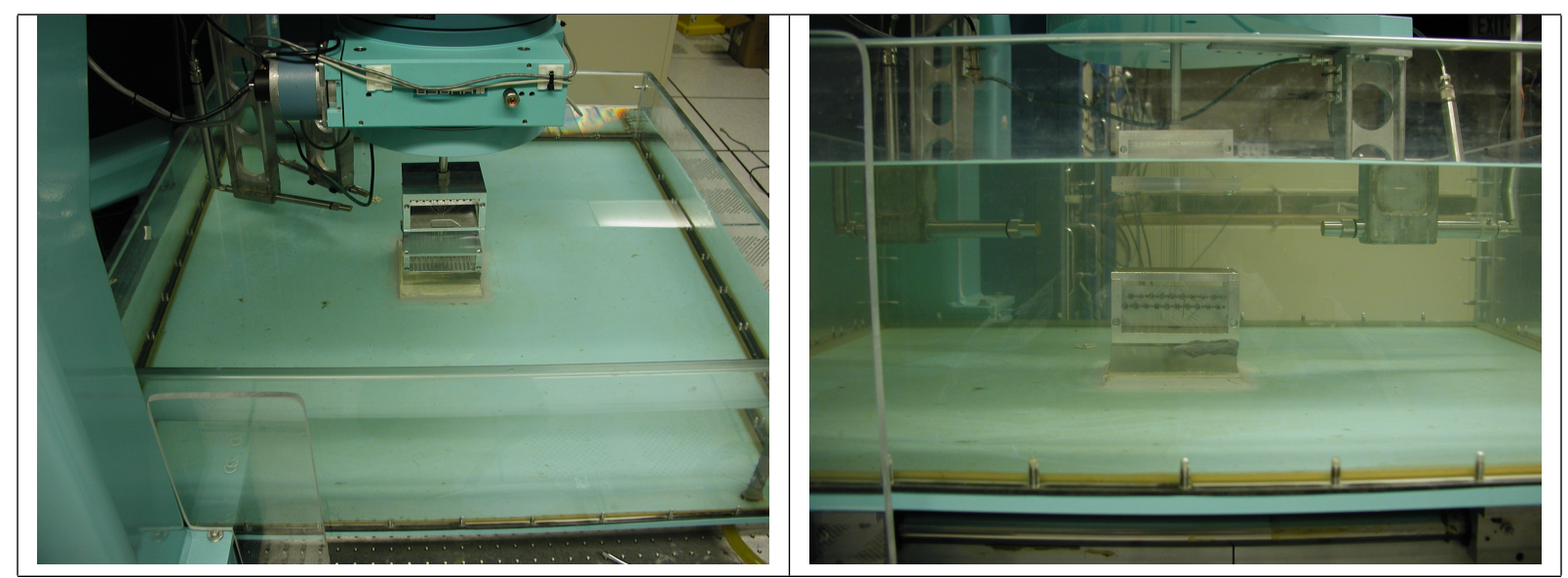

Figure 20: Two views of the wire phantom in the water bath and annular acoustic array. 


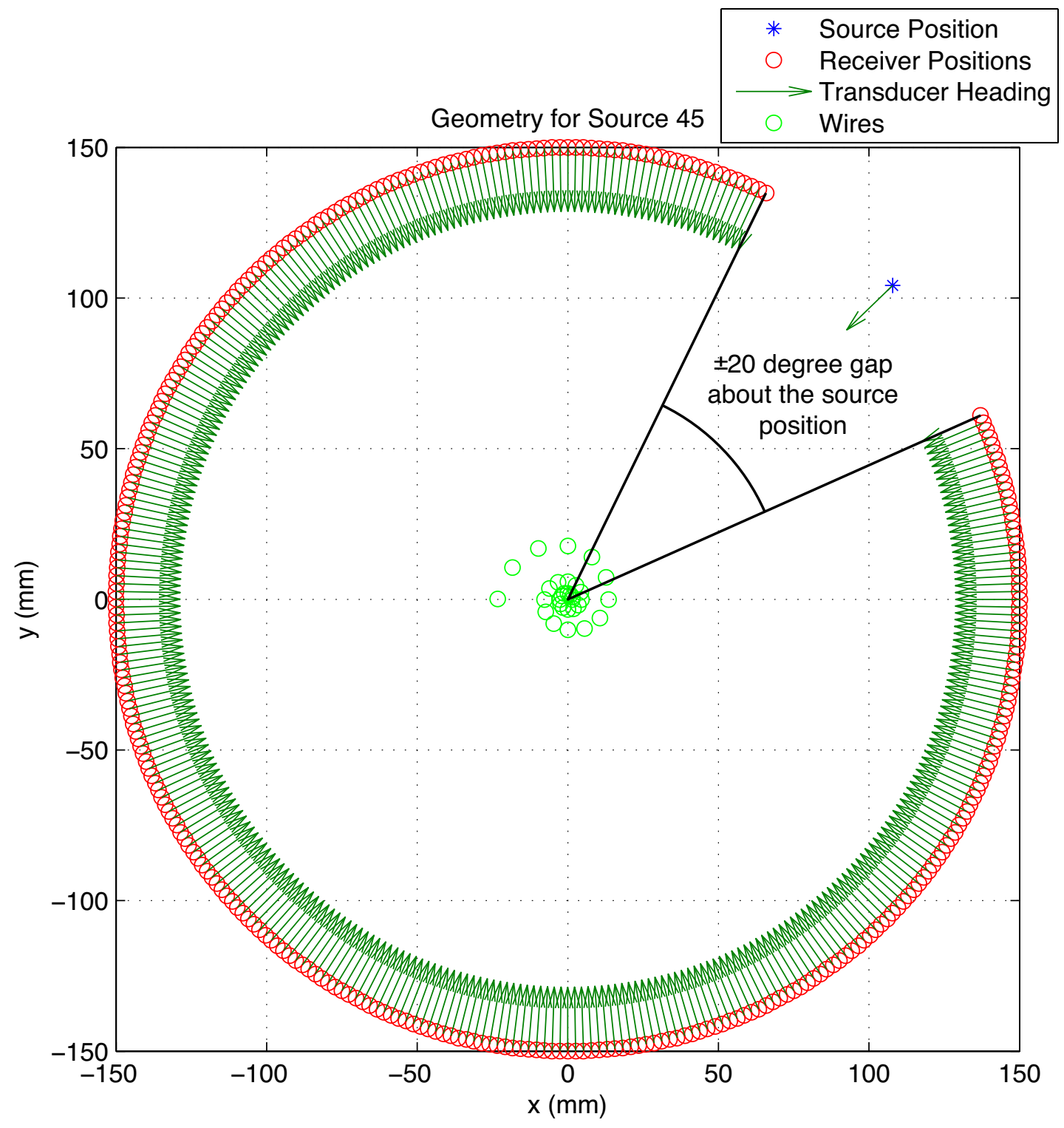

Figure 21: Graphic of the annular array and the \pm 20 degree gap. 


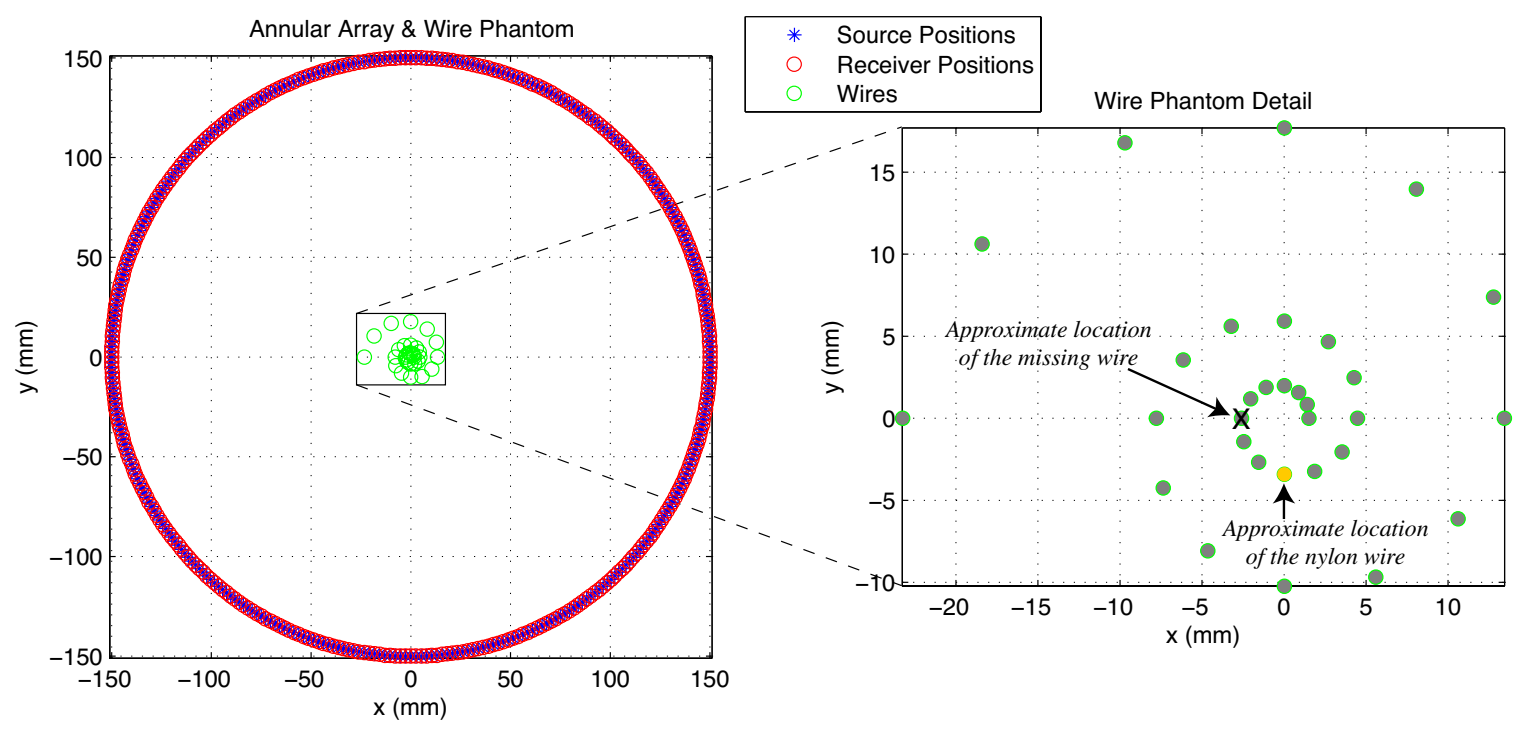

Figure 22: Graphic of the annular array with the wire phantom.

\subsection{Aluminum/Copper Planar Multilayer}

The aluminum/copper planar multilayer of Figure 25 presents the case of data collection in a planar multilayer with the goal of migration in a non-homogeneous background. This models the cases of a defect in an "interior" layer with no "exterior" access or a buried object whose location is unknown and to be determined. Imaging must be performed through the outer layer. A 32 element fully multistatic $5 \mathrm{MHz}$ pulse system was used to probe the structure with the goal of locating the hole or "defect" in the copper layer.

Figure 26 shows a multimonostatic measured response extracted from the full multistatic measurement. The reflections from the $\mathrm{Al} / \mathrm{Cu}$ interface and the bottom of the $\mathrm{Cu}$ layer are prominent. Less so are the multipath reflection between the transducer and the $\mathrm{Al} / \mathrm{Cu}$ interface and the hyberbolic "smile" from the hole in the $\mathrm{Cu}$ layer.

\subsection{Ground Penetrating Radar}

A wide-band ground penetrating radar with a peak pulse frequency of approximately $2.2 \mathrm{GHz}$ collected data multimonostatically ten $\mathrm{cm}$ above a dry loamy sandbox. There were 260 horizontal transducer "pitch/catch" measurement locations. The aluminum pole resolution phantom of Figure 28 was buried approximately six $\mathrm{cm}$ below the surface. Figure 29 presents the measured backscattered time series. The refractive index of the sand was empirically estimated to be approximately 2.8 . Thus, the peak pulse wavelength in the sand was approximately $3 \times 10^{8} / 2.2 \times 10^{9} / 2.8 \approx 5 \mathrm{~cm}$.

\subsection{Aluminum Block as a Model for Offset Vertical Seismic Profiling}

Vertical seismic profiling (VSP) is a geophysical measurement technique in which a receiver chain is inserted into a borehole to receive fields resulting from a surface source. In offset vertical seismic profiling (OVSP), the surface source(s) is(are) offset from the borehole. A graphical depiction of OVSP is shown in Figure 30.

An aluminum block with two drilled holes was used in an OVSP proof-of-principle experiment. A multistatic $5 \mathrm{MHz}$ pulse system was used to measure the holes "buried" within the block. The source array consisted of 64 elements and the receiving array, placed orthogonally to the source array on an adjacent edge, had 30 . The experiment and migrated reconstruction are presented in Figure 31. 
30 Steel/1 Nylon MSDM for Source 35
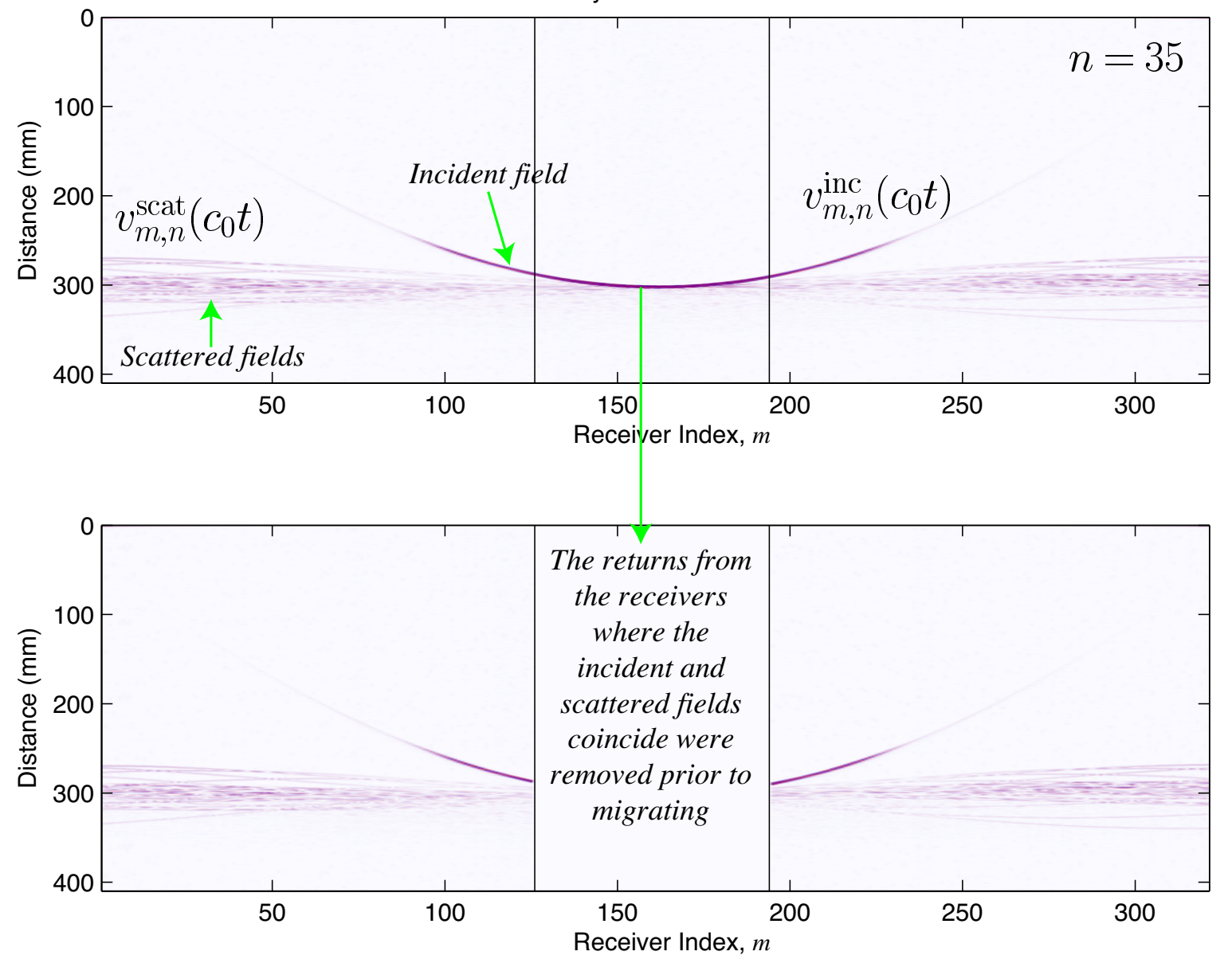

Figure 23: Typical measured data time series. 


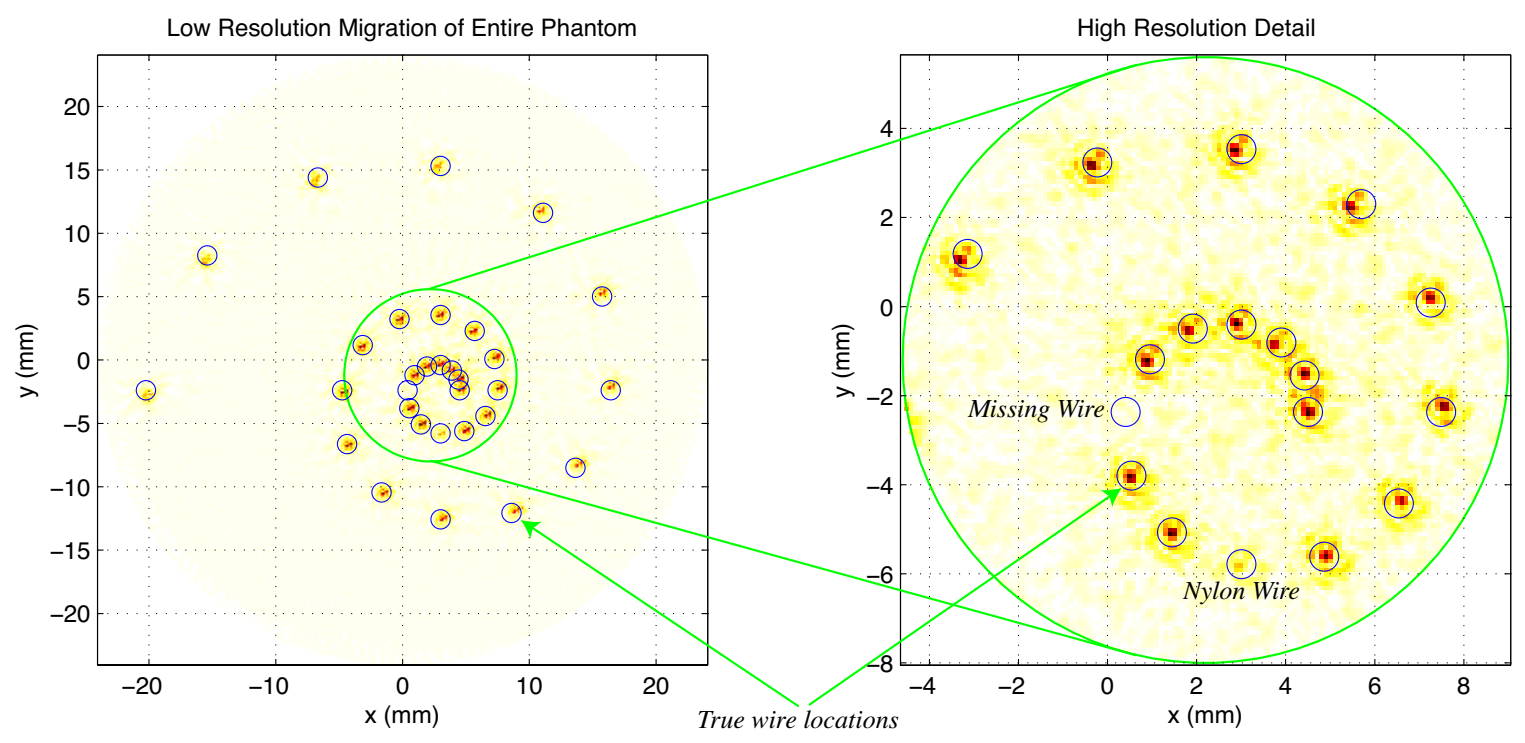

Figure 24: Migrated 30 steel/1 nylon using a background velocity of $1501.00 \mathrm{~m} / \mathrm{s}$ and a device radius of $150.60 \mathrm{~mm}$. The blue circles indicate the "As-Designed" wire locations. Note the missing wire and the location of the nylon wire.

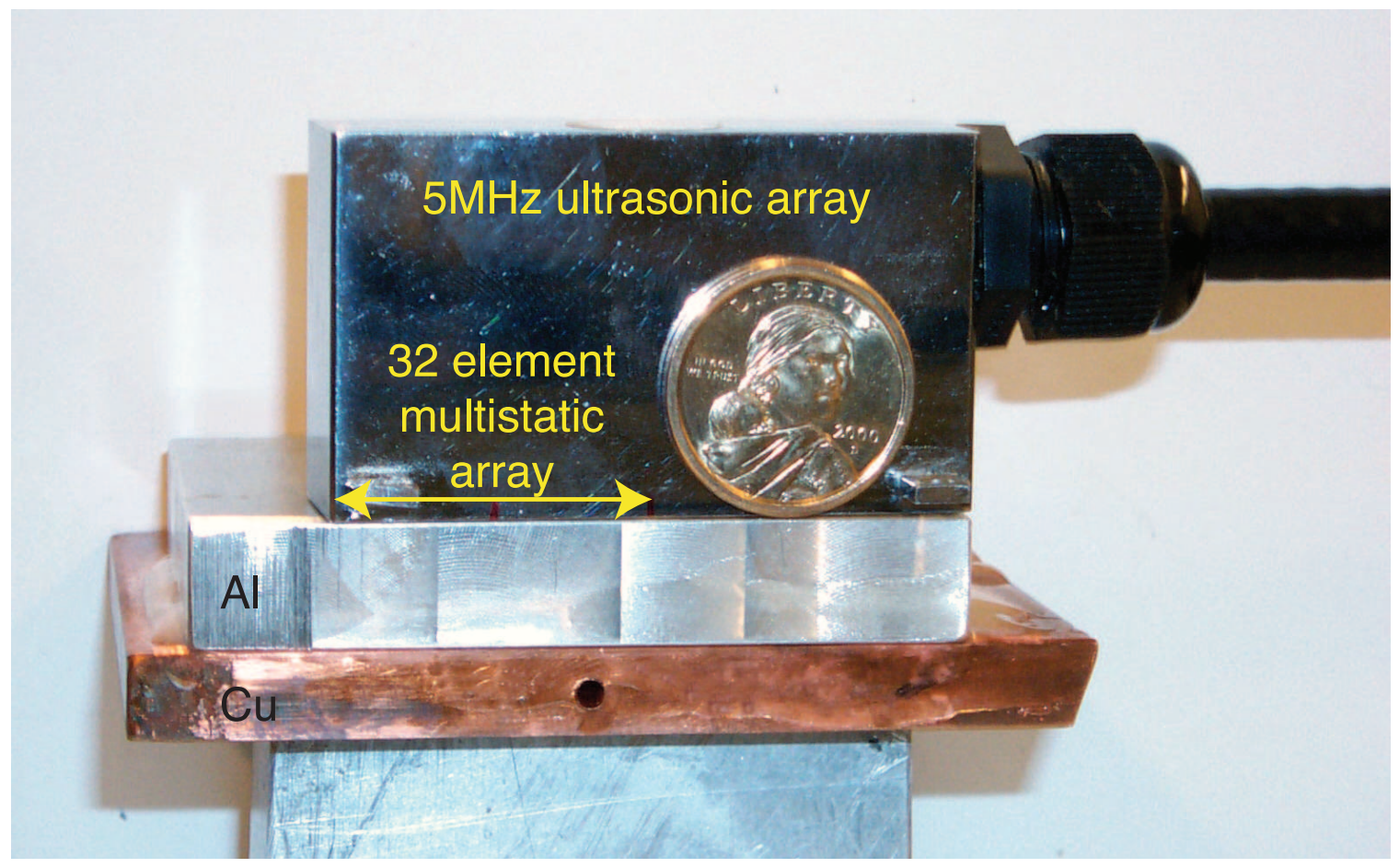

Figure 25: Al/Cu planar multilayer. 


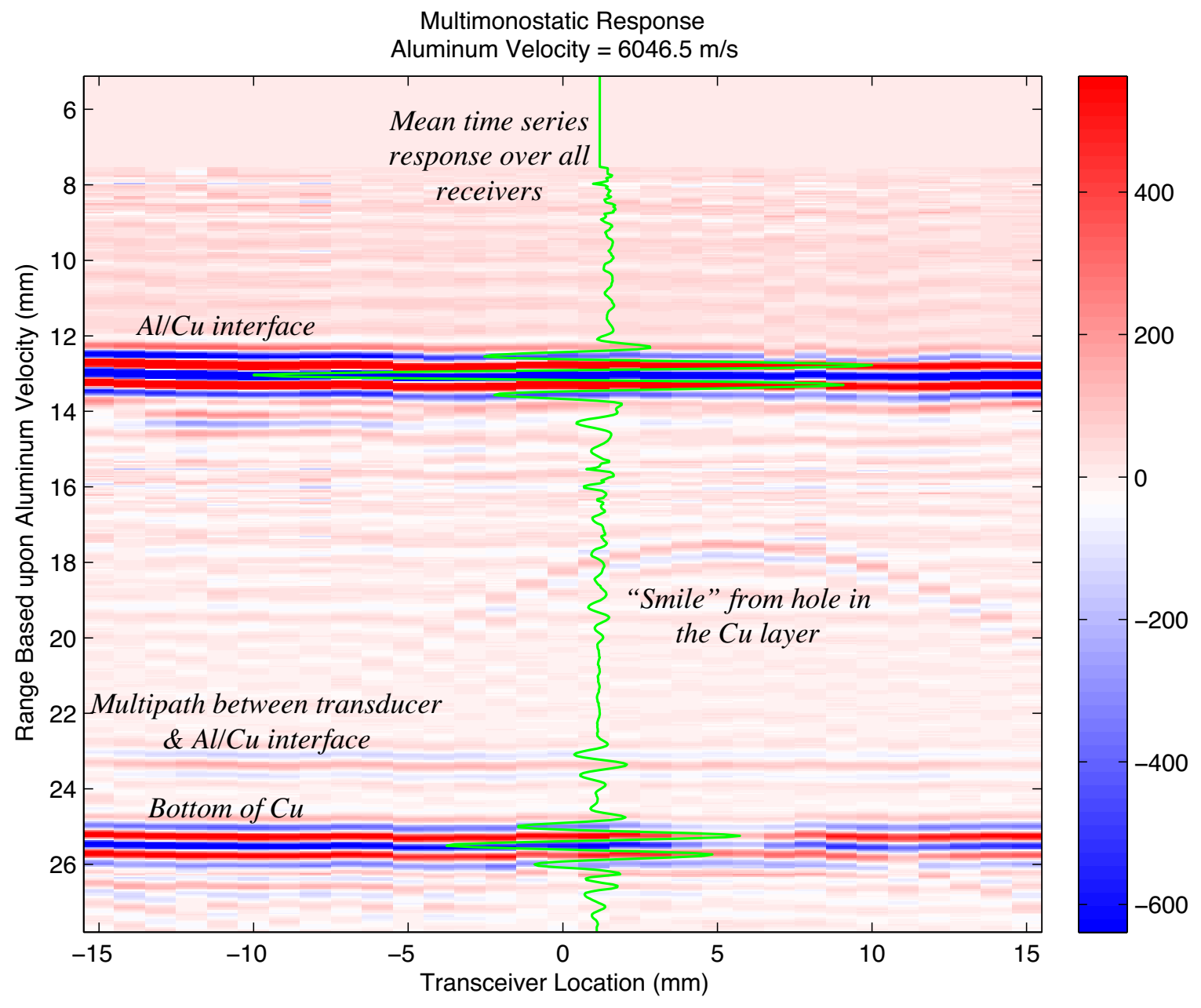

Figure 26: Al/Cu multimonostatic time series response extracted from the full multistatic measurement. The reflections from the $\mathrm{Al} / \mathrm{Cu}$ interface and the bottom of the Cu layer are prominent. Less so are the multipath reflection between the transducer and the Al/Cu interface and the hyberbolic "smile" from the hole in the Cu layer. 


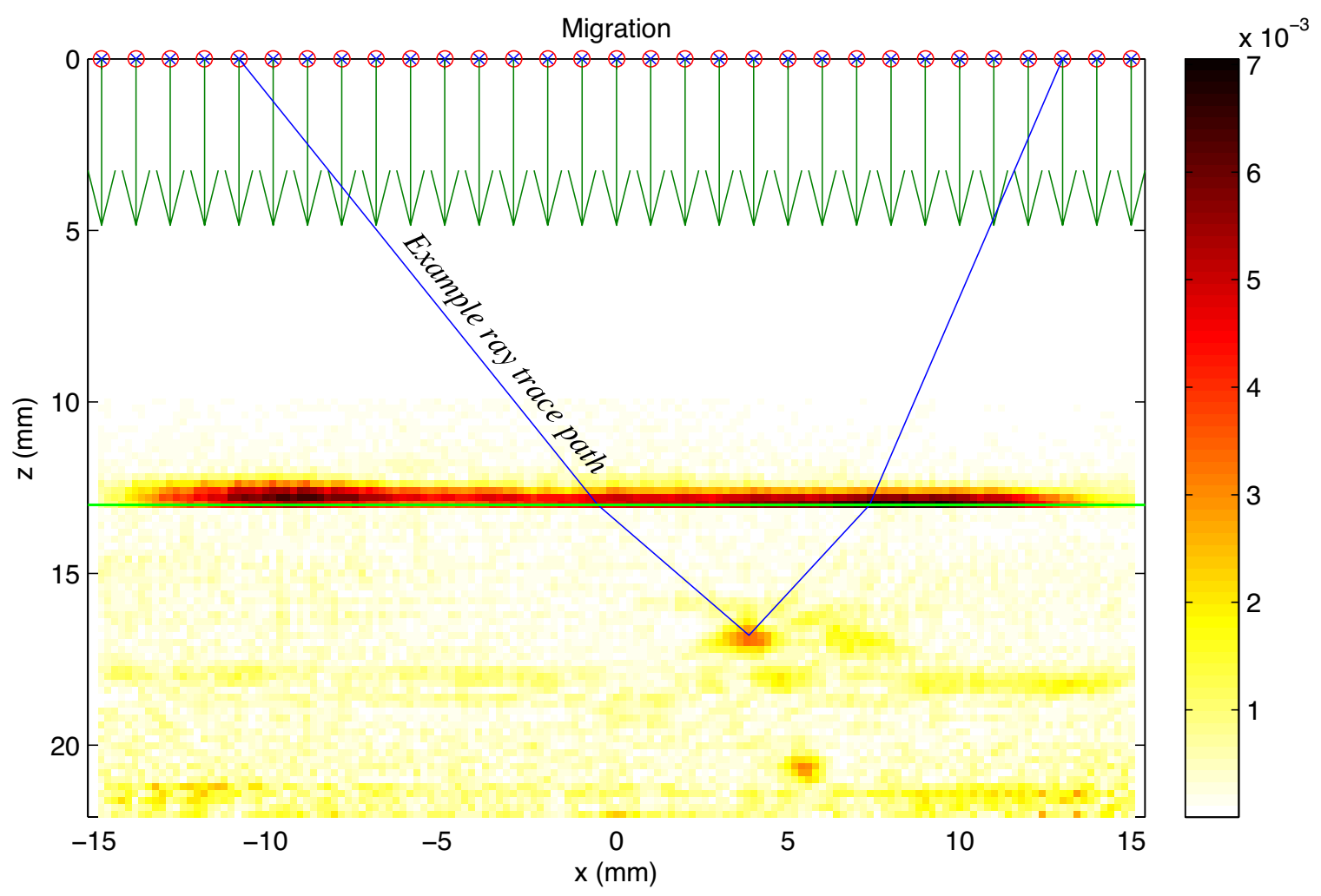

Figure 27: Al/Cu migration with a sample ray trace to the hole in the Cu layer.

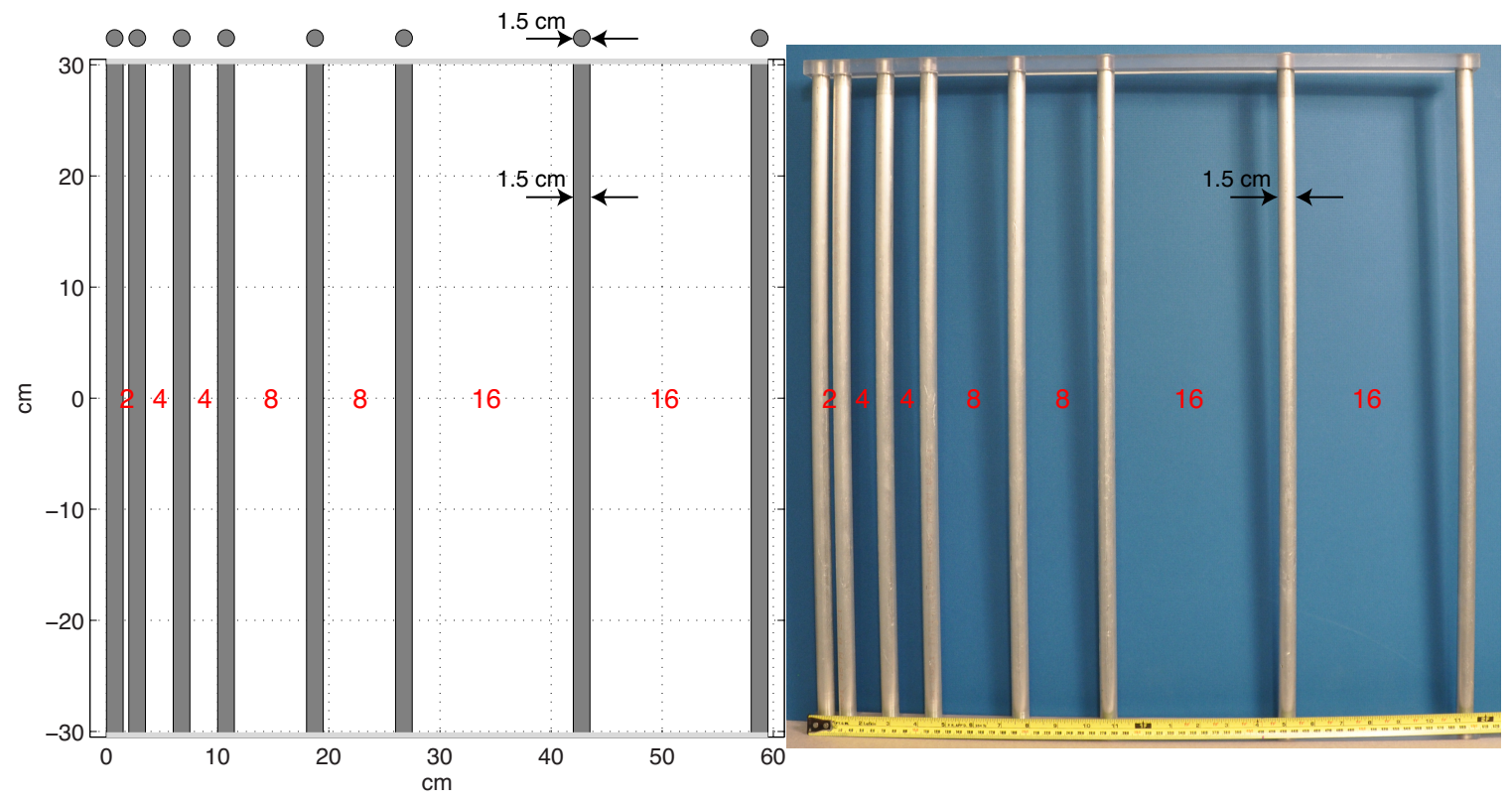

Figure 28: A resolution phantom consisting of eight 1.5 centimeter diameter aluminum poles. 


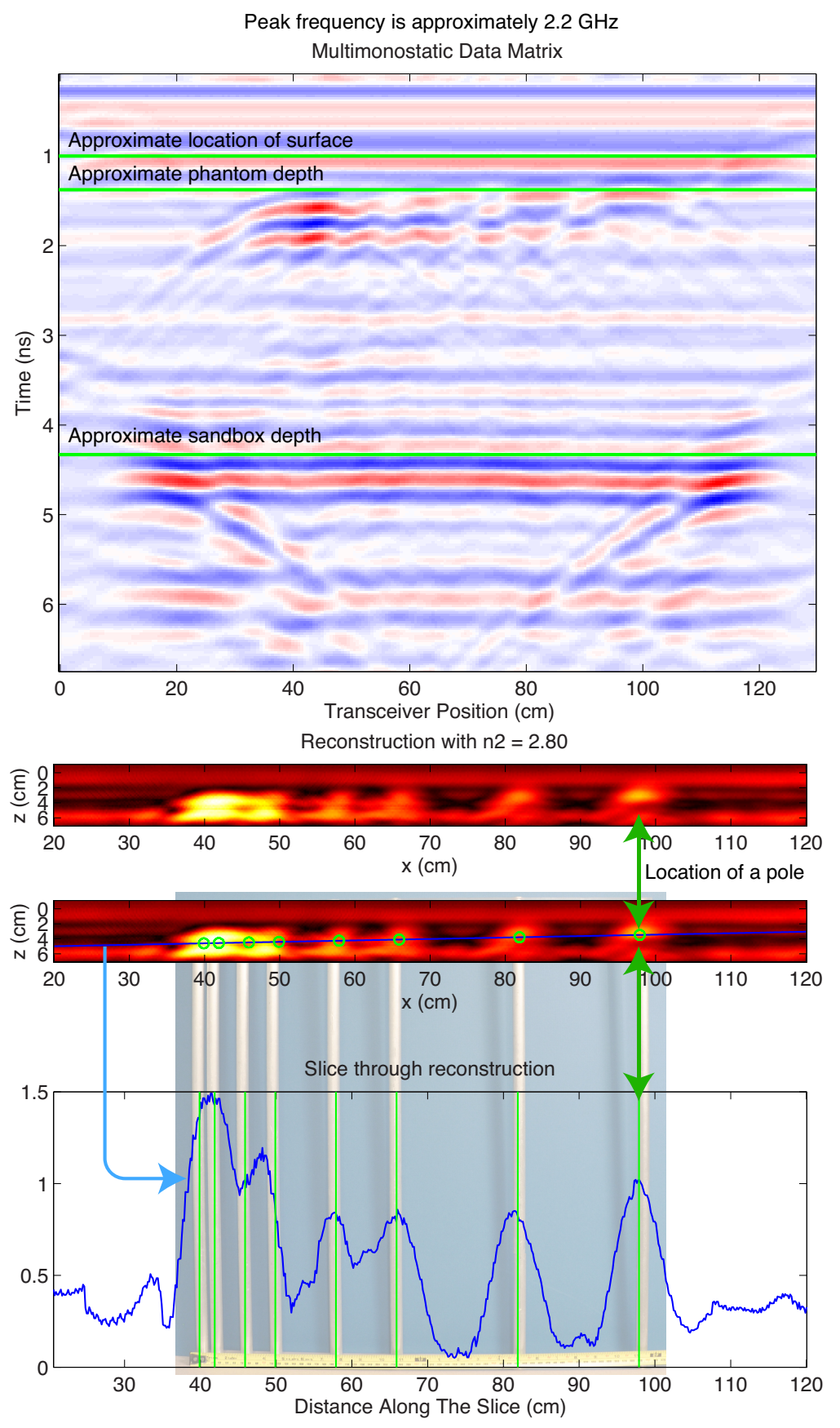

Figure 29: The resolution phantom buried approximately six cm deep in dry, loamy sand. The wide band electromagnetic transducer was approximately $10 \mathrm{~cm}$ above the surface. The center frequency was $2.2 \mathrm{GHz}$. 


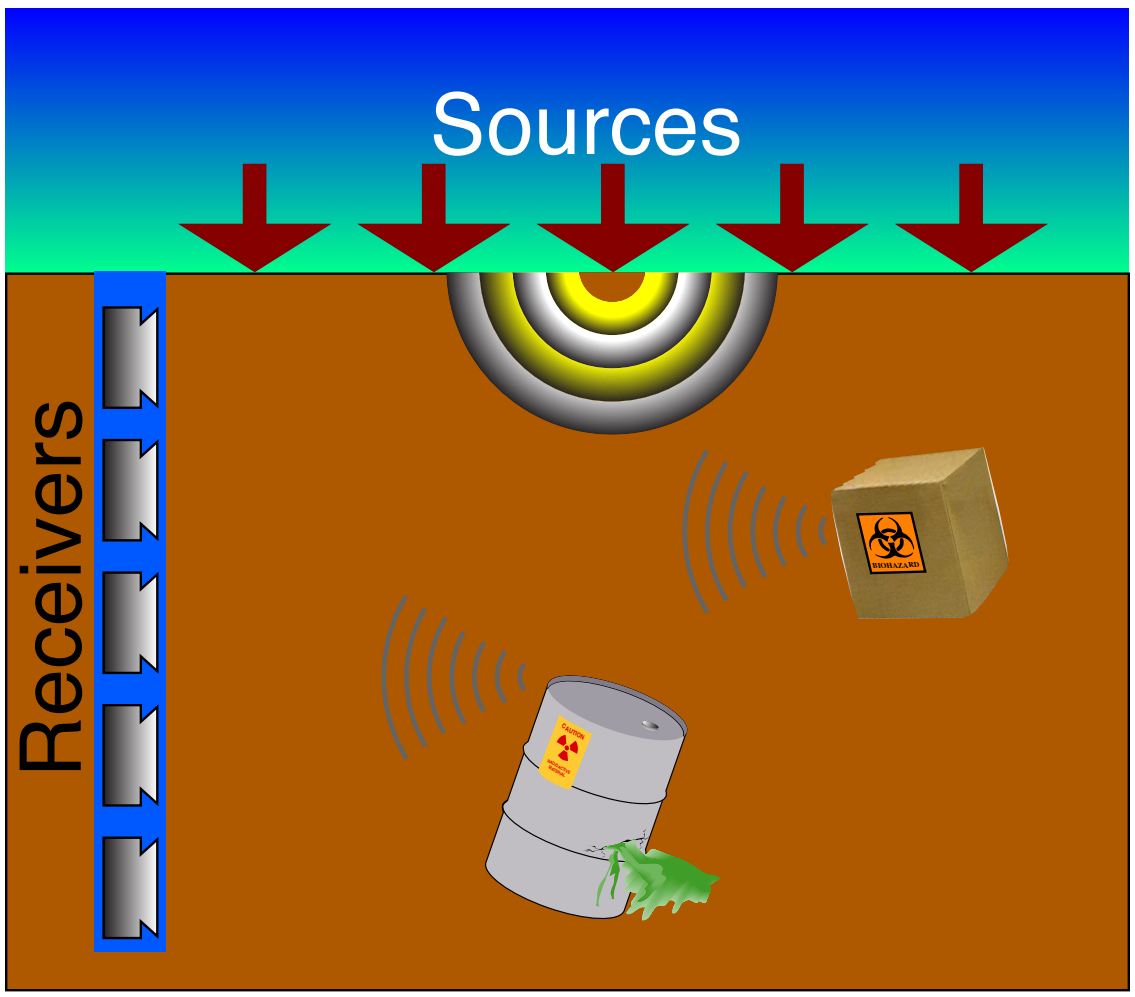

Figure 30: Graphic depicting offset vertical seismic profiling. A receiver chain is inserted into a borehole. In succession, surface sources are excited and the resultant fields measured at the receivers. 


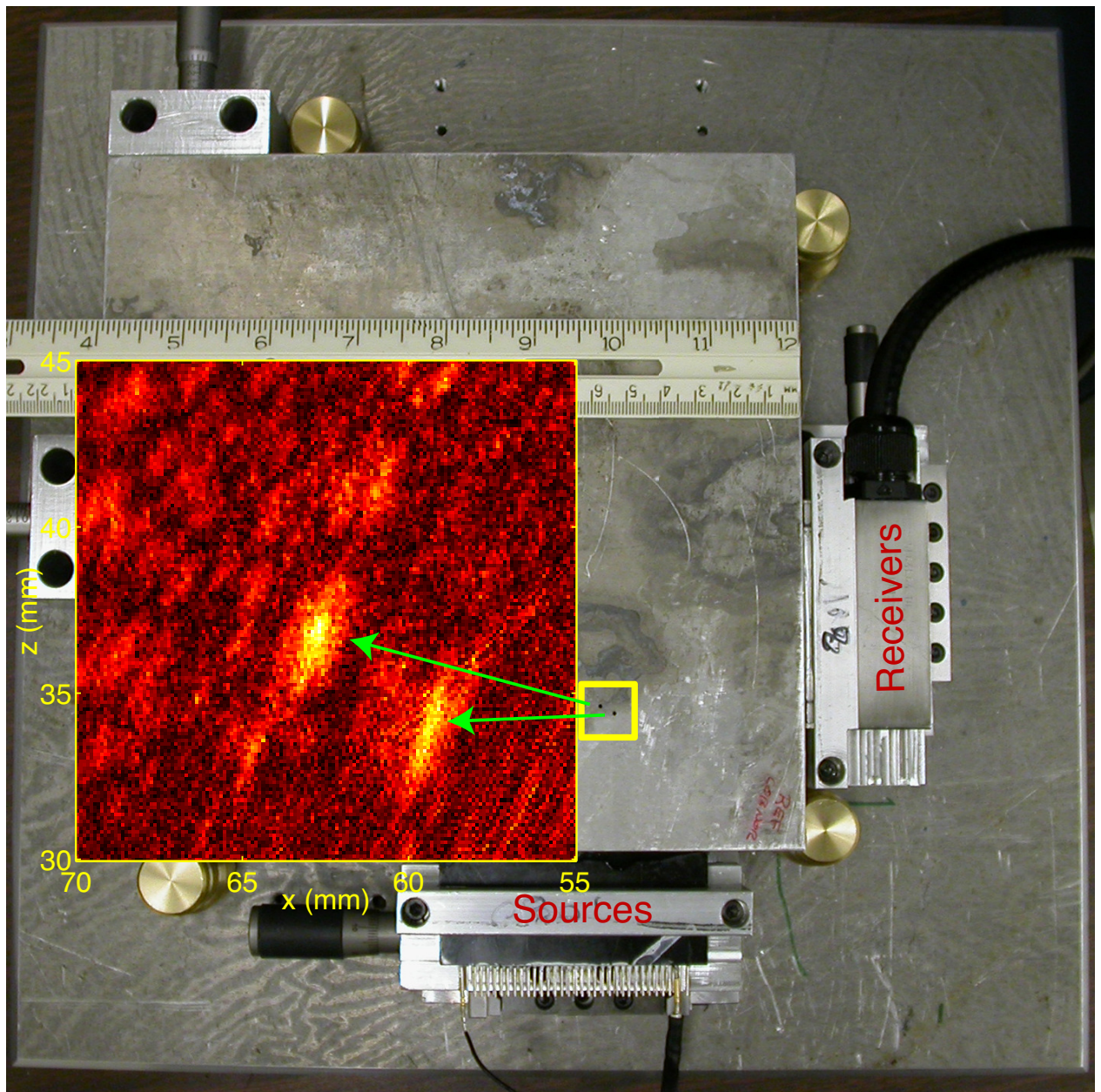

Figure 31: Aluminum block experiment used to model offset vertical seismic profiling. The source array is coupled to the "surface" and the receiving array is "placed" in a "vertical borehole". 


\section{A Standard Fourier Transforms}

\section{A.1 Forward in Time}

$$
\psi(\mathbf{r}, \omega)=\int_{-\infty}^{\infty} d t \psi(\mathbf{r}, t) e^{i \omega t}
$$

\section{A.2 Inverse in Time}

$$
\psi(\mathbf{r}, t)=\frac{1}{2 \pi} \int_{-\infty}^{\infty} d \omega \psi(\mathbf{r}, \omega) e^{-i \omega t}
$$

\section{A.3 Forward in Space}

$$
\psi(\mathbf{k}, \omega)=\int_{-\infty}^{\infty} d \mathbf{r} \psi(\mathbf{r}, \omega) e^{-i \mathbf{k} \cdot \mathbf{r}}
$$

\section{A.4 Inverse in Space}

$$
\psi(\mathbf{r}, \omega)=\frac{1}{(2 \pi)^{3}} \int_{-\infty}^{\infty} d \mathbf{k} \psi(\mathbf{k}, \omega) e^{i \mathbf{k} \cdot \mathbf{r}}
$$

\section{A.5 Planar Fourier Transform Pair}

$$
\begin{aligned}
\psi\left(\mathbf{k}_{\perp}, z, \omega\right) & =\int_{-\infty}^{\infty} d \mathbf{r}_{\perp} \psi\left(\mathbf{r}_{\perp}, z, \omega\right) e^{-i \mathbf{k}_{\perp} \cdot \mathbf{r}_{\perp}}, \\
\psi\left(\mathbf{r}_{\perp}, z, \omega\right) & =\frac{1}{(2 \pi)^{(n-1)}} \int_{-\infty}^{\infty} d \mathbf{k}_{\perp} \psi\left(\mathbf{k}_{\perp}, z, \omega\right) e^{i \mathbf{k}_{\perp} \cdot \mathbf{r}_{\perp}},
\end{aligned}
$$

where $n$ is the dimensionality of the problem, either 2 or 3 .

\section{A.6 Closure in Time}

$$
\begin{aligned}
\psi(\mathbf{r}, t) & =\frac{1}{2 \pi} \int_{-\infty}^{\infty} d \omega \int_{-\infty}^{\infty} d \tau \psi(\mathbf{r}, \tau) e^{i \omega \tau} e^{-i \omega t} \\
& =\frac{1}{2 \pi} \int_{-\infty}^{\infty} d \tau \psi(\mathbf{r}, \tau) \underbrace{\int_{-\infty}^{\infty} d \omega e^{-i \omega(t-\tau)}}_{2 \pi \delta(t-\tau)}
\end{aligned}
$$

\section{A.7 Convolution}

$$
\begin{aligned}
f(t) & =\int_{-\infty}^{\infty} d \omega G(\omega) H(\omega) e^{-i \omega t} \\
& =\int_{-\infty}^{\infty} d \omega\left[\int_{-\infty}^{\infty} d t_{1} g\left(t_{1}\right) e^{i \omega t_{1}}\right]\left[\int_{-\infty}^{\infty} d t_{2} h\left(t_{2}\right) e^{i \omega t_{2}}\right] e^{-i \omega t} \\
& =\int_{-\infty}^{\infty} d t_{1} g\left(t_{1}\right) \int_{-\infty}^{\infty} d t_{2} h\left(t_{2}\right) \int_{-\infty}^{\infty} d \omega e^{-i \omega\left(t-t_{1}-t_{2}\right)}
\end{aligned}
$$




$$
\begin{aligned}
& =2 \pi \int_{-\infty}^{\infty} d t_{1} g\left(t_{1}\right) \int_{-\infty}^{\infty} d t_{2} h\left(t_{2}\right) \delta\left(t-t_{1}-t_{2}\right) \\
& =2 \pi \int_{-\infty}^{\infty} d t_{1} g\left(t_{1}\right) h\left(t-t_{1}\right)
\end{aligned}
$$




\section{B Measurement System Operating Modes}

There are many measurement system operating modes or data collection methods. The common ones are

Monostatic : A transceiver remains fixed in space, $\mathbf{R}_{\mathrm{xvr}} \equiv$ constant. An incident field is emitted and any backscattered fields are measured. This is known as "pulse/echo" operation. The data are represented by $v^{\text {scat }}(t)$.

Multimonostatic : A monostatic transceiver performs pulse/echo measurements at successive spatial locations indicated by $\left\{\mathbf{R}_{n}^{\mathrm{xvr}}\right\}_{n=1}^{N_{\mathrm{xvr}}}$.

Bistatic : A source at a fixed location, $\mathbf{R}^{\text {src }} \equiv$ constant, emits an incident field. A spatially separated receiver at a fixed location, $\mathbf{R}^{\mathrm{rcv}} \equiv$ constant $\neq \mathbf{R}^{\mathrm{src}}$, measures any scattered fields. The incident field may also be measured. This is known as "pitch/catch" operation. The data are represented by $v^{\text {scat }}(t)$.

Multistatic : Successively, multiple spatially separated sources, located at $\left\{\mathbf{R}_{n}^{\text {src }}\right\}_{n=1}^{N_{\text {src }}}$, emit incident fields. Scattered fields are measured simultaneously at multiple spatially separated receivers, located at $\left\{\mathbf{R}_{m}^{\mathrm{rcv}}\right\}_{m=1}^{N_{\mathrm{rcv}}}$. Sources and receivers may share common spatial locations. The data are represented by $v_{m n}^{\text {scat }}(t)$.

Multibistatic : A bistatic pair with a fixed offset perform pitch/catch operations at successive spatial locations, $\left\{\mathbf{R}_{n}^{\text {src }}, \mathbf{R}_{n}^{\text {rcv }}\right\}_{n=1}^{N_{\text {xvr }}}$ where $\left|\mathbf{R}_{n}^{\text {rcv }}-\mathbf{R}_{n}^{\text {src }}\right| \equiv$ constant. The data are represented by $v_{n}^{\text {scat }}(t)$.

Variable Offset Multibistatic : A multibistatic pair perform pitch/catch operations with one transceiver fixed while the other operates at successive spatial locations. The locations are described by one of

- $\mathbf{R}^{\mathrm{src}} \equiv$ constant with multiple receiver positions, $\left\{\mathbf{R}_{m}^{\mathrm{rcv}}\right\}_{m=1}^{N_{\mathrm{rcv}}}$, or

- $\mathbf{R}^{\text {rcv }} \equiv$ constant with multiple source positions, $\left\{\mathbf{R}_{n}^{\mathrm{src}}\right\}_{n=1}^{N_{\text {src }}}$.

The data are represented by $v_{m n}^{\text {scat }}(t)$ where $m$ can be a function of $n$ or vice-versa. 


\section{Three Dimensional Planar Layer Ray Trace}

Given a source (ray start) point, $\mathbf{R}_{n}^{\text {src }}=\left(x_{n}, y_{n}, z_{n}\right)$, and a focal point, $\mathbf{r}_{\mathrm{f}}=\left(x_{\mathrm{f}}, y_{\mathrm{f}}, z_{\mathrm{f}}\right)$, determine the surface intercept point, $\mathbf{p}=\left(x_{\mathrm{p}}, y_{\mathrm{p}}, z_{\mathrm{p}}\right)$, constraining the rays by Snell's Law. The solution is a three step process:

1. Rotate and translate the $x y$-coordinate system so that the source and focal points share a common $y$-plane. That is, align the points along a common plane perpendicular to the surface. Use the source point as the new origin;

2. Solve Snell's Law for the surface intercept point;

3. Reverse the translation and rotation.

The planar rotation angle, $\phi$, is given by

$$
\phi=\tan ^{-1}\left(\frac{y_{\mathrm{f}}-y_{n}}{x_{\mathrm{f}}-x_{n}}\right) .
$$

The rotation matrix and translation vector are

$$
\begin{aligned}
U & =\left[\begin{array}{ccc}
\cos \phi & \sin \phi & 0 \\
-\sin \phi & \cos \phi & 0 \\
0 & 0 & 1
\end{array}\right]=\left[\begin{array}{ccc}
\frac{x_{\mathrm{f}}-x_{n}}{\left|\mathbf{r}_{\mathrm{f} \perp}-\mathbf{R}_{n \perp}^{\text {src }}\right|} & \frac{y_{\mathrm{f}}-y_{n}}{\left|\mathbf{r}_{\mathrm{f} \perp}-\mathbf{R}_{n \perp}^{\text {src }}\right|} & 0 \\
-\frac{y_{\mathrm{f}_{\perp}}-y_{n}}{\left|\mathbf{r}_{\mathrm{f} \perp}-\mathbf{R}_{n \perp}^{\text {src }}\right|} & \frac{x_{\mathrm{f}}-x_{n}}{\left|\mathbf{r}_{\mathrm{f} \perp}-\mathbf{R}_{n \perp}^{\text {src }}\right|} & 0 \\
0 & 0 & 1
\end{array}\right], \\
T & =\left[\begin{array}{c}
x_{n} \\
y_{n} \\
z_{n}
\end{array}\right],
\end{aligned}
$$

respectively, and $\left|\mathbf{r}_{\mathrm{f} \perp}-\mathbf{R}_{n \perp}^{\mathrm{src}}\right| \equiv \sqrt{\left(x_{\mathrm{f}}-x_{n}\right)^{2}+\left(y_{\mathrm{f}}-y_{n}\right)^{2}}$. In the new coordinate system, $\mathbf{R}_{n}^{\mathrm{src}^{\prime}} \equiv(0,0,0)$. The focal point is

$$
\begin{aligned}
\mathbf{r}_{\mathrm{f}}^{\prime}=U\left(\mathbf{r}_{\mathrm{f}}^{T}-T\right)= & {\left[\begin{array}{ccc}
\frac{x_{\mathrm{f}}-x_{n}}{\left|\mathbf{r}_{\mathrm{f} \perp}-\mathbf{R}_{n \perp}^{\text {src }}\right|} & \frac{y_{\mathrm{f}}-y_{n}}{\left|\mathbf{r}_{\mathrm{f}}-\mathbf{R}_{n \perp}^{\operatorname{src}}\right|} & 0 \\
-\frac{y_{\mathrm{f}}-y_{n}}{\left|\mathbf{r}_{\mathrm{f} \perp}-\mathbf{R}_{n \perp}^{\operatorname{src}}\right|} & \frac{x_{\mathrm{f}}-x_{n}}{\left|\mathbf{r}_{\mathrm{f} \perp}-\mathbf{R}_{n \perp}^{\operatorname{src}}\right|} & 0 \\
0 & 0 & 1
\end{array}\right]\left[\begin{array}{c}
x_{\mathrm{f}}-x_{n} \\
y_{\mathrm{f}}-y_{n} \\
z_{\mathrm{f}}-z_{n}
\end{array}\right] } \\
= & {\left[\begin{array}{c}
\frac{\left(x_{\mathrm{f}}-x_{n}\right)^{2}+\left(y_{\mathrm{f}}-y_{n}\right)^{2}}{\mid \mathbf{r}_{\mathrm{f} \perp}-\mathbf{R}_{n \perp}^{\mathrm{scc} \mid}} \\
0 \\
z_{\mathrm{f}}-z_{n}
\end{array}\right], }
\end{aligned}
$$

where the primes indicate the source and focal points are represented in the rotated coordinate system. The source and the focal point are now in the $y^{\prime} \equiv 0$ plane. In the new (reduced) coordinate system, the source, focal, and intercept points are expressed as,

$$
\begin{aligned}
\mathbf{R}_{n}^{\text {src' }} & =\left[x_{n}^{\prime}, 0, z_{n}^{\prime}\right]^{T}, \\
\mathbf{r}_{\mathrm{f}}^{\prime} & =\left[x_{\mathrm{f}}^{\prime}, 0, z_{\mathrm{f}}^{\prime}\right]^{T}, \\
\mathbf{p}^{\prime} & =\left[x_{\mathrm{p}}^{\prime}, 0, z_{\mathrm{p}}^{\prime}\right]^{T} .
\end{aligned}
$$

To reduce clutter, the primes will be dropped but the reader is reminded the following derivation is performed in the translated and rotated coordinate system. Referring to Figure 17, the following geometric relationships are derived,

$$
\begin{aligned}
\sin \theta_{1} & =\frac{x_{p}-x_{n}}{\sqrt{\left(x_{p}-x_{n}\right)^{2}+\left(z_{p}-z_{n}\right)^{2}}} \\
\sin \theta_{2} & =\frac{x_{\mathrm{f}}-x_{p}}{\sqrt{\left(x_{\mathrm{f}}-x_{p}\right)^{2}+\left(z_{\mathrm{f}}-z_{p}\right)^{2}}}
\end{aligned}
$$


Substitute these identities into Eqn. (100),

$$
n_{1} \frac{x_{\mathrm{p}}-x_{n}}{\sqrt{\left(x_{\mathrm{p}}-x_{n}\right)^{2}+\left(z_{\mathrm{p}}-z_{n}\right)^{2}}}=n_{2} \frac{x_{\mathrm{f}}-x_{\mathrm{p}}}{\sqrt{\left(x_{\mathrm{f}}-x_{\mathrm{p}}\right)^{2}+\left(z_{\mathrm{f}}-z_{\mathrm{p}}\right)^{2}}},
$$

and cast the expression into the form of a function of the unknown $x_{\mathrm{p}}$ to be minimized,

$$
f\left(x_{\mathrm{p}}\right)=\frac{1}{2}\left[n_{1}^{2}\left(x_{\mathrm{p}}-x_{n}\right)^{2}\left(\left(x_{\mathrm{f}}-x_{\mathrm{p}}\right)^{2}+\left(z_{\mathrm{f}}-z_{\mathrm{p}}\right)^{2}\right)-n_{2}^{2}\left(x_{\mathrm{f}}-x_{\mathrm{p}}\right)^{2}\left(\left(x_{\mathrm{p}}-x_{n}\right)^{2}+\left(z_{\mathrm{p}}-z_{n}\right)^{2}\right)\right]^{2},
$$

which may be solved numerically. For reference, its first derivative is

$$
\frac{d}{d x_{\mathrm{p}}} f\left(x_{\mathrm{p}}\right)=2 \sqrt{2 f\left(x_{\mathrm{p}}\right)}\left[n_{1}^{2} x_{\mathrm{p}}\left(2 x_{\mathrm{p}}^{2}-3 x_{\mathrm{p}} x_{\mathrm{f}}+x_{\mathrm{f}}^{2}+\left(z_{\mathrm{p}}-z_{\mathrm{f}}\right)^{2}\right)-n_{2}^{2}\left(x_{\mathrm{p}}-x_{\mathrm{f}}\right)\left(2 x_{\mathrm{p}}^{2}-x_{\mathrm{p}} x_{\mathrm{f}}+z_{\mathrm{p}}^{2}\right)\right] .
$$

Given the solution, $\mathbf{p}^{\prime}=\left(x_{\mathrm{p}}^{\prime}, z_{\mathrm{p}}^{\prime}\right)$, in the rotated and translated frame, the intercept point in the original coordinate system is

$$
\begin{aligned}
& \mathbf{p}=U^{-1} \mathbf{p}^{\prime}+T, \\
& =\left[\begin{array}{ccc}
\frac{x_{\mathrm{f}}-x_{n}}{\left|\mathbf{r}_{\mathrm{f}}-\mathbf{R}_{n \perp}^{\text {src }}\right|} & -\frac{y_{\mathrm{f}}-y_{n}}{\left|\mathbf{r}_{\mathrm{f}}-\mathbf{R}_{n \perp}^{\text {src }}\right|} & 0 \\
\frac{y_{\mathrm{f}}-y_{n}}{\left|\mathbf{r}_{\mathrm{f} \perp}-\mathbf{R}_{n \perp}^{\text {src }}\right|} & \frac{x_{\mathrm{f}}-x_{n}}{\left|\mathbf{r}_{\mathrm{f} \perp}-\mathbf{R}_{n \perp}^{\text {src }}\right|} & 0 \\
0 & 0 & 1
\end{array}\right]\left[\begin{array}{c}
x_{\mathrm{p}}^{\prime} \\
0 \\
z_{\mathrm{p}}^{\prime}
\end{array}\right]+\left[\begin{array}{c}
x_{n} \\
y_{n} \\
z_{n}
\end{array}\right], \\
& =\left[\begin{array}{c}
\frac{x_{\mathrm{f}}-x_{n}}{\left|\mathbf{r}_{\mathrm{f} \perp}-\mathbf{R}_{n \perp}^{\text {src }}\right|} x_{\mathrm{p}}^{\prime}+x_{n} \\
\frac{y_{\mathrm{f}}-y_{n}}{\left|\mathbf{r}_{\mathrm{f} \perp}-\mathbf{R}_{n \perp}^{\text {src }}\right|} x_{\mathrm{p}}^{\prime}+y_{n} \\
z_{\mathrm{p}}^{\prime}+z_{n}
\end{array}\right]
\end{aligned}
$$

The process is repeated for the rays from $\mathbf{r}$ to $\mathbf{R}_{m}^{\mathrm{rcv}}$ through $\mathbf{q}$. From this the range scales and delays are calculated as

$$
\begin{aligned}
\alpha_{\mathrm{p}, n} & =\left|\mathbf{p}-\mathbf{R}_{n}^{\mathrm{src}}\right|, \\
\alpha_{\mathrm{f}, \mathrm{p}} & =\left|\mathbf{r}_{\mathrm{f}}-\mathbf{p}\right|, \\
\alpha_{\mathrm{q}, \mathrm{f}} & =\left|\mathbf{q}-\mathbf{r}_{\mathrm{f}}\right|, \\
\alpha_{m, \mathrm{q}} & =\left|\mathbf{R}_{m}^{\mathrm{rcv}}-\mathbf{q}\right| .
\end{aligned}
$$

The total range scaling and delay are,

$$
\begin{aligned}
\alpha_{m f n} & =\left(\alpha_{\mathrm{p}, n}+\alpha_{m, \mathrm{q}}\right) \times\left(\alpha_{\mathrm{f}, \mathrm{p}}+\alpha_{\mathrm{q}, \mathrm{f}}\right) \\
\tau_{m \mathrm{f} n} & =\frac{\left(\alpha_{\mathrm{p}, n}+\alpha_{m, \mathrm{q}}\right)}{c_{0} / n_{1}}+\frac{\left(\alpha_{\mathrm{f}, \mathrm{p}}+\alpha_{\mathrm{q}, \mathrm{f}}\right)}{c_{0} / n_{2}}
\end{aligned}
$$

respectively.

\section{C.1 Addressing Numerical Concerns}

Care must be taken when implementing Eqns. 114 and 125 when the transceiver and focal points exist in the same $x-y$ plane, that is when

$$
\mathbf{R}_{n \perp}^{\mathrm{src}} \equiv \mathbf{r}_{\mathrm{f} \perp}
$$

To avoid division by zero, it is best to express the equations as

$$
\begin{aligned}
& \mathbf{r}_{\mathrm{f}}^{\prime}= {\left[\begin{array}{c}
\left(x_{\mathrm{f}}-x_{n}\right) \cos \phi+\left(y_{\mathrm{f}}-y_{n}\right) \sin \phi \\
0 \\
z_{\mathrm{f}}-z_{n}
\end{array}\right], } \\
& \mathbf{p}=\left[\begin{array}{c}
x_{\mathrm{p}}^{\prime} \cos \phi+x_{n} \\
x_{\mathrm{p}}^{\prime} \sin \phi+y_{n} \\
z_{\mathrm{p}}^{\prime}+z_{n}
\end{array}\right]
\end{aligned}
$$




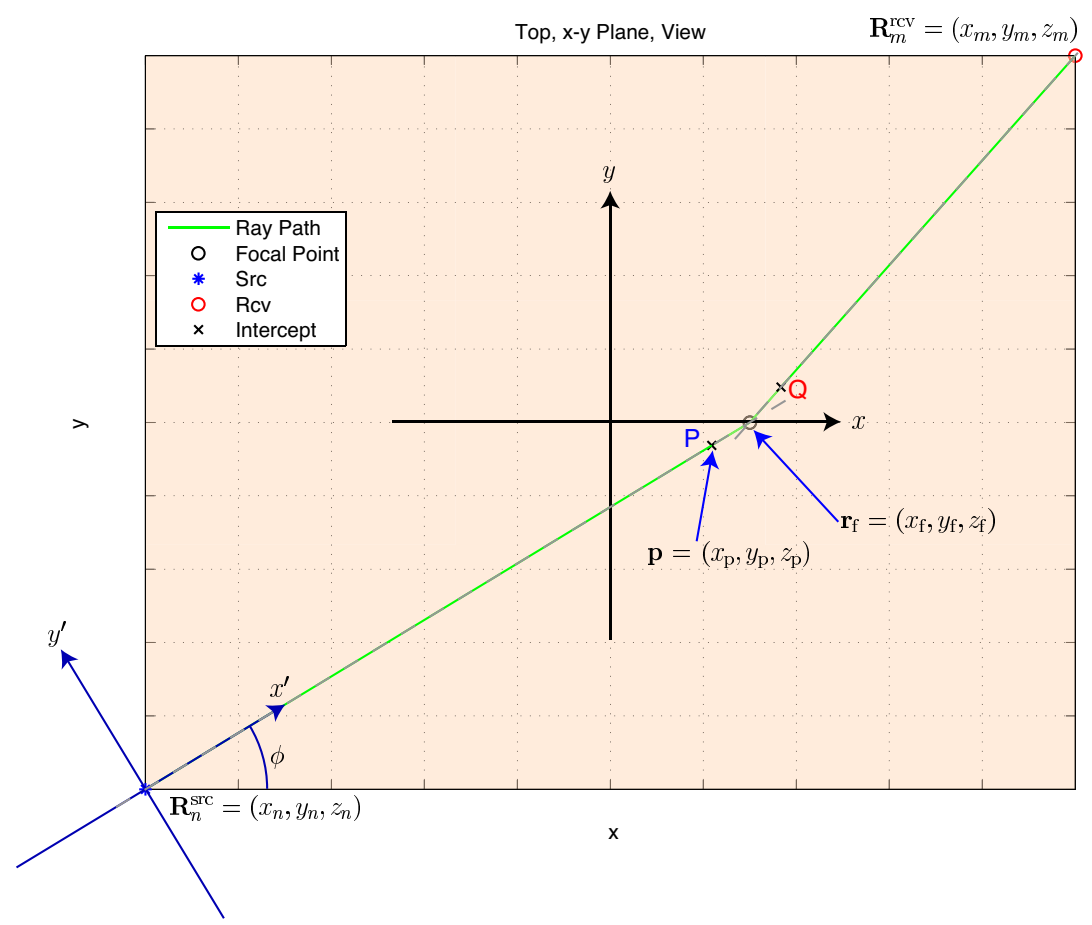

(a) Horizontal, $x y$-plane, view of the three dimensional ray trace.

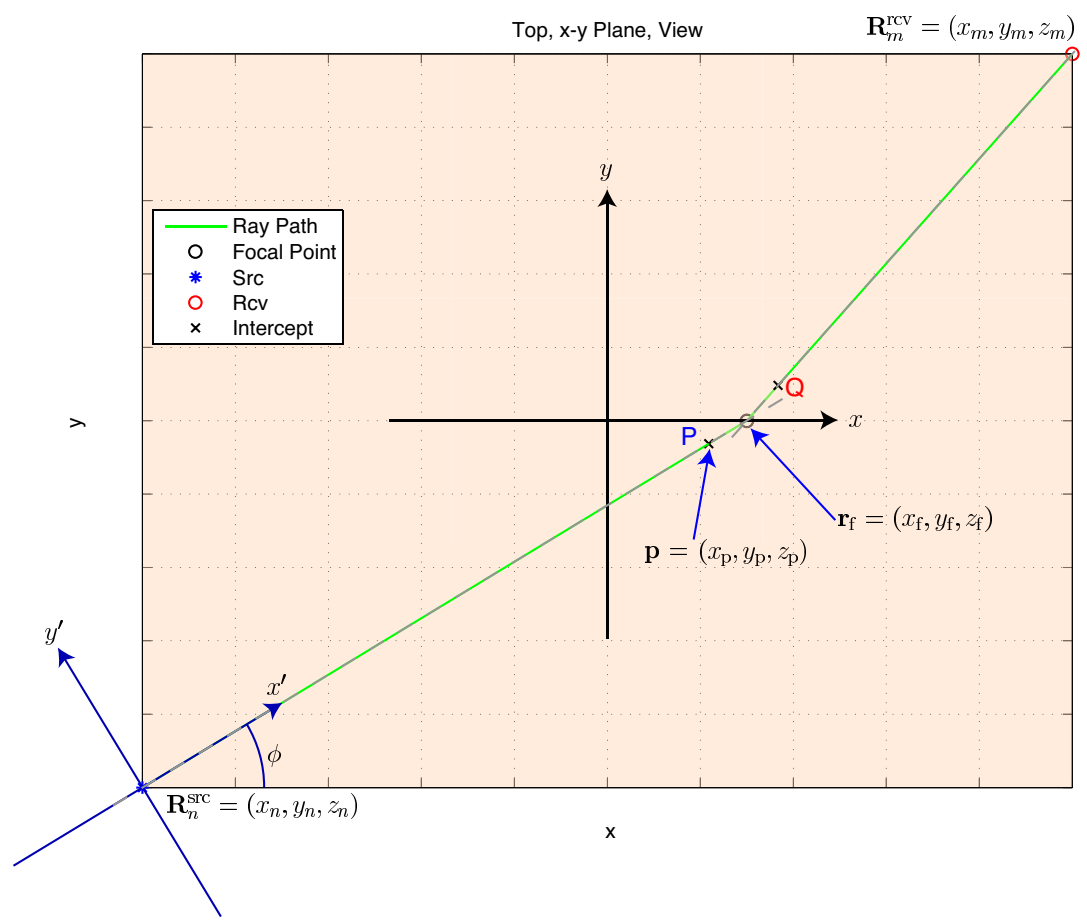

(b) Vertical slice, $x^{\prime} z$-plane, view of the ray trace.

Figure 32: Horizontal and vertical slice views of the ray trace of Figure 16. (a) The horizontal plane showing the translated and rotated coordinate system in which the ray trace is solved. (b) The vertical slice along the rotated $x^{\prime}$ axis. 


\section{Hilbert Transform}

From MATLAB's perspective the Hilbert transform of a function $s(t)$ is defined as the inverse Fourier transform of the real Fourier components of the transform of $s(t)$. Mathematically, it is

$$
\begin{aligned}
S(\omega) & =\mathcal{F}\{s(t)\}=\int d t s(t) e^{i \omega 1}, \\
\mathcal{H}[s(t)] & =\mathcal{F}^{-1}\{2 \Theta(\omega) S(\omega)\},
\end{aligned}
$$

where $\Theta(\omega)$ is the Heavyside or unit step function,

$$
\Theta(x)=\left\{\begin{array}{ll}
0 & x<0 \\
\frac{1}{2} & x=0 \\
1 & x>0
\end{array} .\right.
$$

The Fourier transform of the unit step function is

$$
\mathcal{F}\{\Theta(t)\}=\pi \delta(\omega)-\frac{i}{\omega} .
$$

By reciprocity, we have

$$
\mathcal{F}^{-1}\{\Theta(\omega)\}=\pi \delta(t)-\frac{i}{t} .
$$

Thus, we have

$$
\mathcal{H}[s(t)]=2 \pi s(t)-\frac{2 i}{t} * s(t) .
$$

Rigorously, the Hilbert transform of a signal is the convolution term,

$$
\mathcal{H}\{s(t)\}=\frac{1}{\pi} \mathrm{P} \int_{-\infty}^{\infty} d \tau \frac{s(\tau)}{t-\tau} .
$$




\section{E Problem Solving Methodology}

A successful method of problem solving is to:

1. Perform a literature search;

2. Develop the theory and a forward analytical model;

3. Develop a forward numerical model or simulation (preferably using existing verified and validated codes);

4. Develop the inversion algorithm or solution and test it against the data of (3);

5. Work with the experimentalist(s) and/or data collection technician(s) to develop controlled experiments, if possible. Test the inversion algorithm of (4) against these data, and feed back to (2);

6. Proceed onto the actual problem in the real environment (with noise, measurement system limitations, etc.). Process these data with the inversion algorithm of (4) and feed back to (2);

7. Develop and test metrics. 


\section{F MATLAB Codes}

The delay, scale, and sum beamforming codes are divided into three categories

1. Those which compute the delay, $\tau_{m \mathrm{ff} n}$, scale, $\alpha_{m \mathrm{fn} n}$, and beam pattern, $b_{m \mathrm{fn} n}$, parameters;

2. Those which apply the parameters and perform the migration;

3. That which performs the ray trace. 


\section{F.1 Migration Parameter Codes}

\section{F.1.1 BFParameters}

Compute the parameters for a beamformer

$[$ delay scale $]=$ BFParameters(Rf, Rxvr, dt, c0)
$[$ delay scale $]=$ BFParameters (Rf, Rsrc, Rrcv, dt, c0)

The first form returns the parameters for a multimonostatic delay, scale, and sum migration. The second form returns the parameters for a multistatic migration.

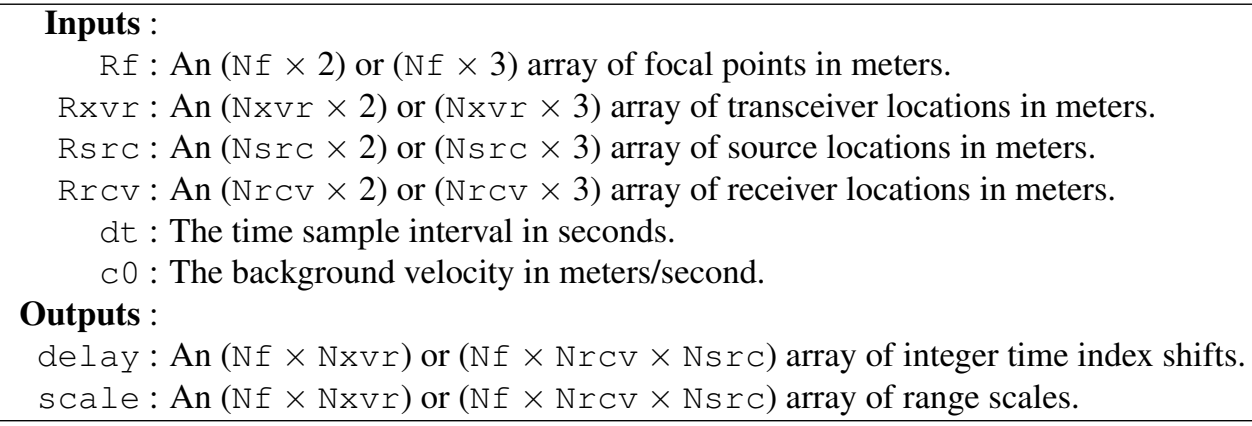




\section{F.1.2 BFParametersBP}

Compute the parameters for a beamformer which imposes a beam pattern

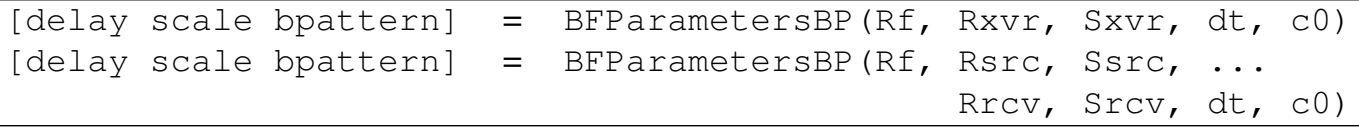

The first form returns the parameters for a multimonostatic delay, scale, and sum migration. The second form returns the parameters for a multistatic migration.

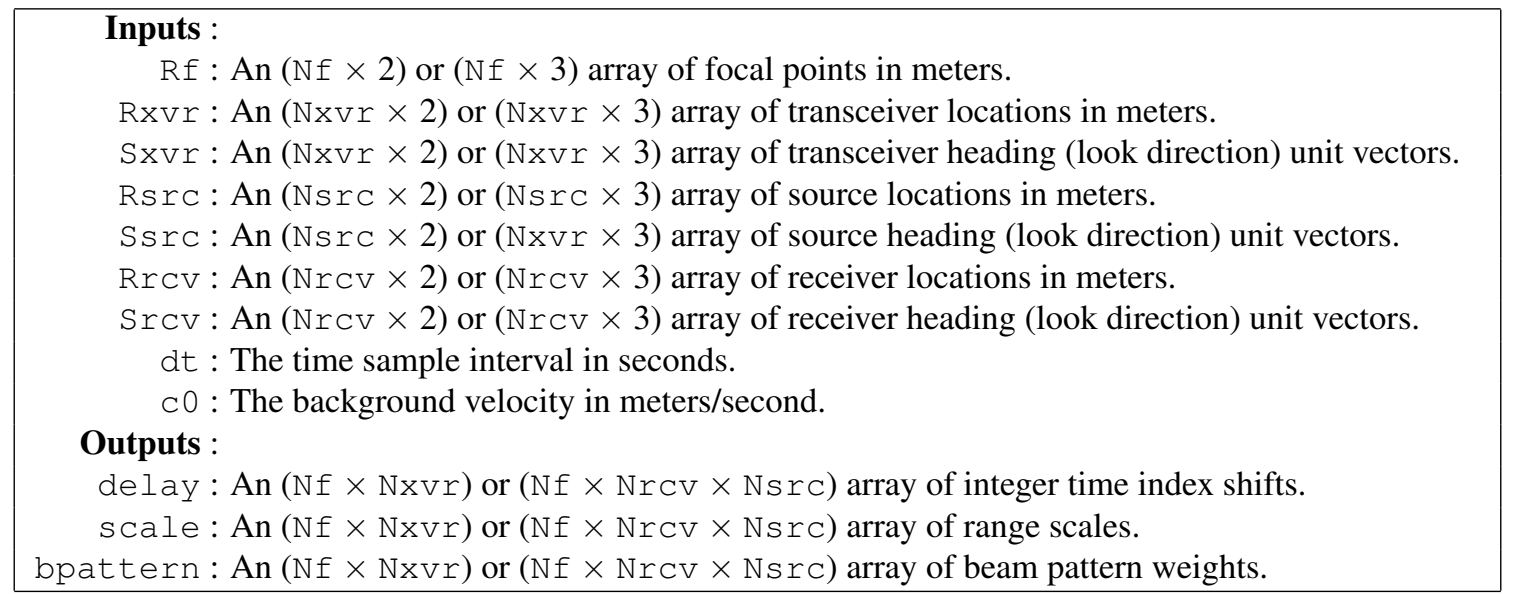




\section{F.1.3 BFParametersBP2L}

Compute the parameters for a two planar layer beamformer which imposes a beam pattern

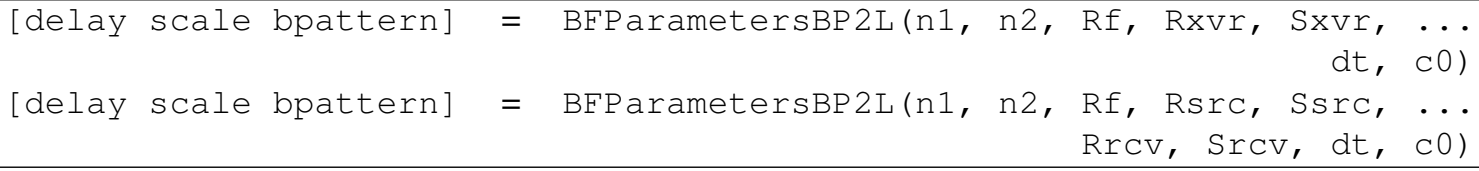

The first form returns the parameters for a multimonostatic delay, scale, and sum migration. The second form returns the parameters for a multistatic migration.

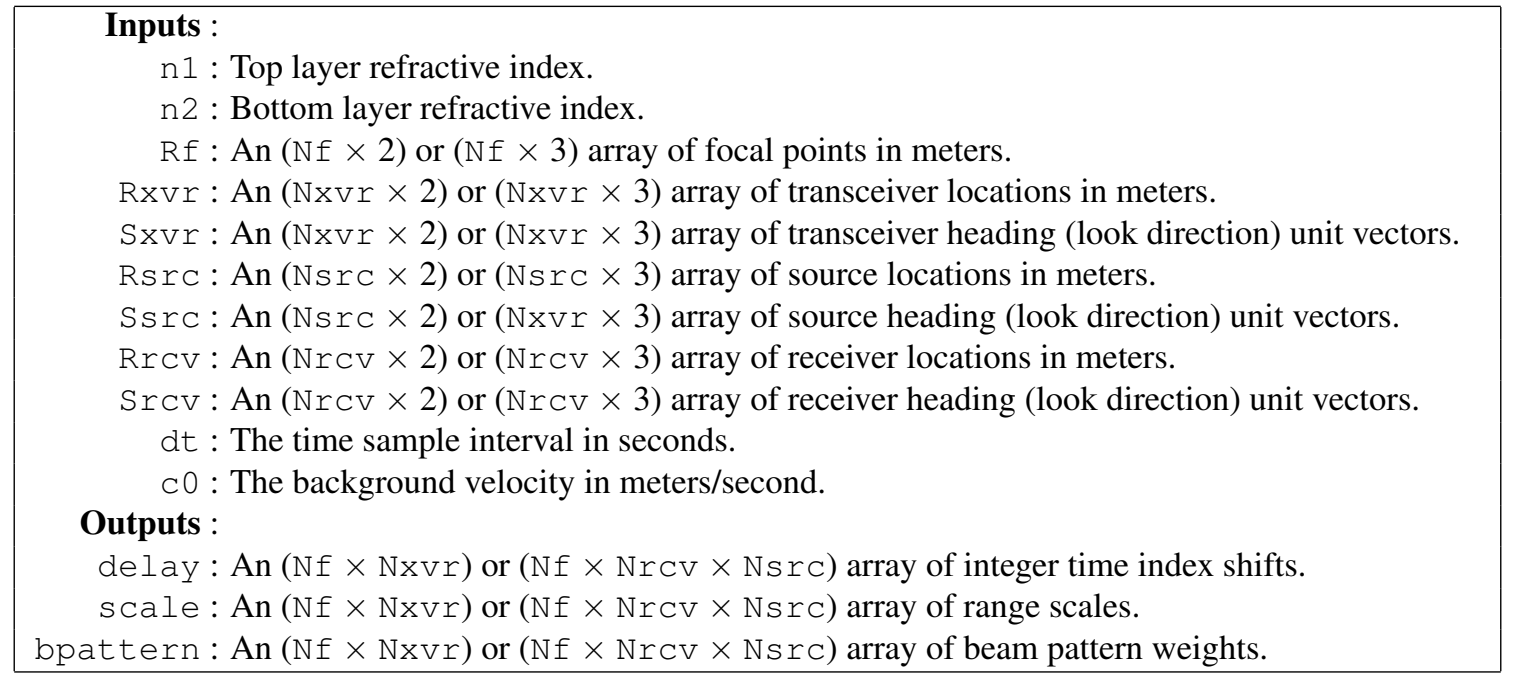




\section{F.2 Migration Computation Codes}

\section{F.2.1 BFCompute}

Apply the parameters for a beamformer

$$
\begin{aligned}
& \mathrm{p}=\text { BFCompute (delay, data, ntx) } \\
& \mathrm{p}=\text { BFCompute (delay, data, ntx, scale) } \\
& \mathrm{p}=\text { BFCompute (delay, data, ntx, scale, bpattern) } \\
& \mathrm{p}=\text { BFCompute (delay, data, ntx, [], bpattern) }
\end{aligned}
$$

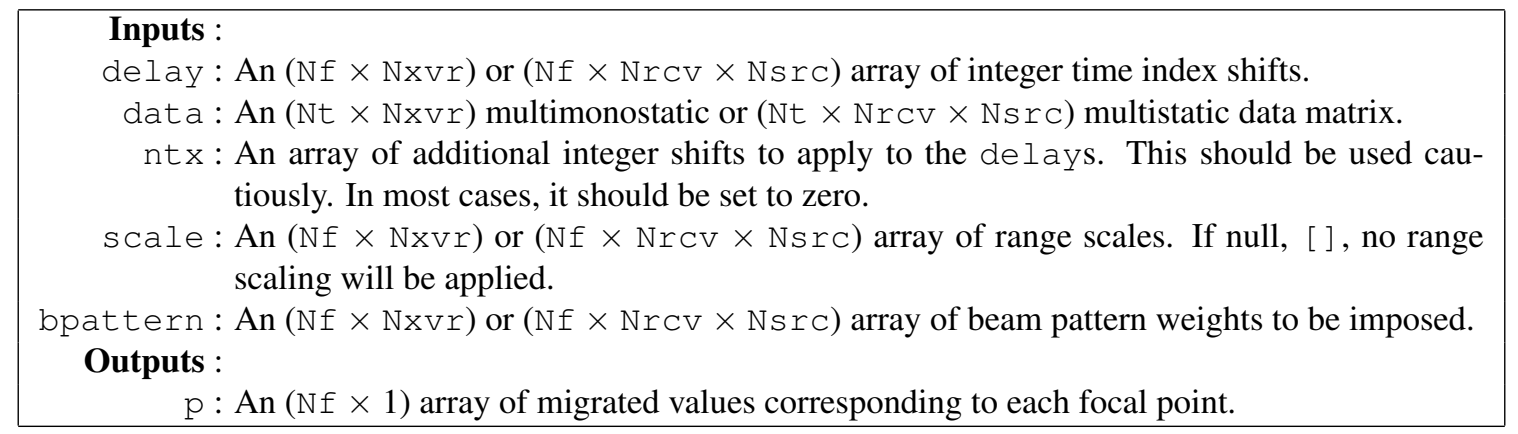

Measurement systems can impose scale factors which modify the physics-based range scale and beam pattern weights, $\alpha_{m \mathrm{fn}}$ and $b_{m \mathrm{f} n}$. Corrections may be applied to these weights in the migration computation code via BFCompute(delay, data, 0, scale.`RangeScaleWeight, bpattern.`BeamPatternWeight) where, for example, RangeScaleWeight is set to 0.25, and BeamPatternWeight is 6.

\section{F.2.2 BFCompute_a}

Apply the parameters for a beamformer where the source and receiver time series within the multistatic data matrix are reversed.

\begin{tabular}{|l}
$\mathrm{p}=$ BFCompute_a (delay, data, ntx) \\
$\mathrm{p}=$ BFCompute_a (delay, data, ntx, scale) \\
$\mathrm{p}=$ BFCompute_a (delay, data, ntx, scale, bpattern) \\
$\mathrm{p}=$ BFCompute_a (delay, data, ntx, [], bpattern)
\end{tabular}

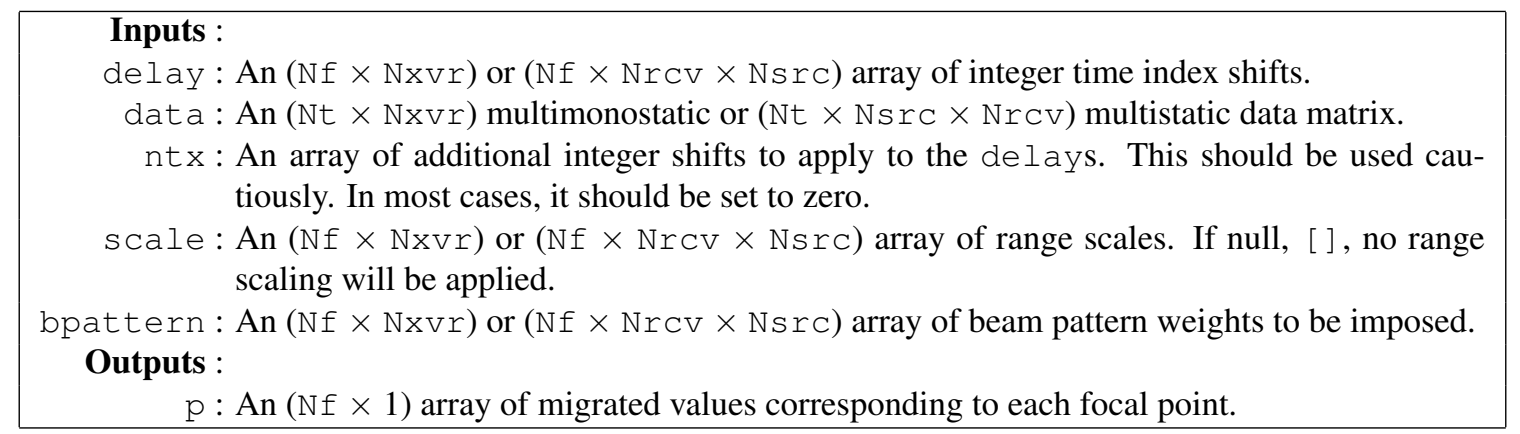

Measurement systems can impose scale factors which modify the physics-based range scale and beam pattern weights, $\alpha_{m f n}$ and $b_{m f n}$. Corrections may be applied to these weights in the migration computation code via BFCompute_\{a\} (delay, data, 0, scale.`RangeScaleWeight, bpattern. ^BeamPatternWeight) where, for example, RangeScaleWeight is set to 0.25, and BeamPatternWeight is 6. 


\section{F.3 Ray Trace Code}

\section{F.3.1 rtpath2pointapprox2L}

Compute the surface intercept given the refractive indices, transceiver location, and focal point location.

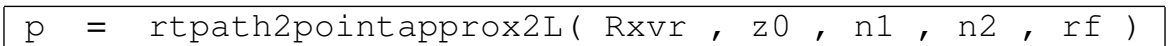

\footnotetext{
Inputs :

Rxvr : A $(1 \times 2)$ or $(1 \times 3)$ element transceiver location in meters.

z 0 : Vertical surface location in meters.

$\mathrm{n} 1$ : Top layer refractive index.

$\mathrm{n} 2$ : Bottom layer refractive index.

$r f$ : A $(1 \times 2)$ or $(1 \times 3)$ element focal point location in meters.
}

\section{Outputs :}

$\mathrm{p}:$ The $x$-direction surface intercept of the ray. 


\section{References}

[1] J. Capon. High-resolution frequency-wavenumber spectrum analysis. Proceedings of the IEEE, 57(8):14081418, August 1969.

[2] H. L. Van Trees. Optimum Array Processing.Part IV of Detection, Estimation, and Modulation Theory. Wiley, 2002. ISBN 0-471-09390-4.

[3] J. F. Claerbout. Fundamentals of Geophysical Data Processing with Applications to Petroleum Prospecting. McGraw-Hill, 1976. ISBN 0-07-011117-0.

[4] N. Bleistein, J. K. Cohen, and J. W. Stockwell Jr. Mathematics of Multidimensional Seismic Imaging, Migration, and Inversion. Interdisciplinary Applied Mathematics. Springer-Verlag, 2001. ISBN 0-387-95061-3.

[5] J. A. Scales. Theory of Seismic Imaging. Samizdat Press, 1994. Available via http://samizdat.mines.edu.

[6] M. Born and E. Wolf. Principles of Optics. Cambridge University Press, 7th edition, 1999. ISBN 0-521-642221.

[7] G. Barton. Elements of Green's Functions and Propagation - Potentials, Diffusion and Waves. Oxford University Press, 1991. ISBN 0-19-851988-6.

[8] J. D. Jackson. Classical Electrodynamics. John Wiley \& Sons, Inc., 3nd edition, 1999. ISBN 0-471-30932-X.

[9] G. Beylkin and M. L. Oristaglio. Distorted-wave born and distorted-wave rytov approximations. Optics Communications, 53(4):213-216, March 1985.

[10] M. Lax. Multiple scattering of waves. Reviews of Modern Physics, 23(4):287-310, October 1951.

[11] L. L. Foldy. The multiple scattering of waves: I. general theory of isotropic scattering by randomly distributed scatterers. Pysical Review, 67(3 \& 4):107-119, February 1945.

[12] M. Lax. The multiple scattering of waves: II. the effective field in dense systems. Physical Review, 85(4):621629, February 1952.

[13] G. H. Golub and C. F. Van Loan. Matrix Computations. The Johns Hopkins University Press, 3nd edition, 1996. ISBN 0-8018-5414-8.

[14] Eugene Hecht. Optics. Addison-Wesley Publishing Company, 2nd edition, 1987. ISBN 0-201-11609-X. 